ELMER MATEUS GENNARO

\title{
Análise da Estabilidade Global de Escoamentos Compressíveis
}




\section{ELMER MATEUS GENNARO}

\section{Análise da Estabilidade Global de Escoamentos Compressíveis}

Tese apresentada à Escola de Engenharia de São Carlos, da Universidade de São Paulo, como parte dos requisitos para obtenção do título de Doutor em Engenharia Mecânica

Àrea de Concentração: Aeronaves

Orientador: Prof. Dr. Marcello A. F. de Medeiros Co-Orientador: Prof. Dr. Vassilios Theofilis

ESTE EXEMPLAR TRATA-SE DA VERSÃO CORRIGIDA.

A VERSÃO ORIGINAL ENCONTRA-SE DISPONÍVEL JUNTO AO DEPARTAMENTO DE ENGENHARIA MECÂNICA DA EESC-USP.

São Carlos 
AUTORIZO A REPRODUÇÃO TOTAL OU PARCIAL DESTE TRABALHO, POR QUALQUER MEIO CONVENCIONAL OU ELETRÔNICO, PARA FINS DE ESTUDO E PESQUISA, DESDE QUE CITADA A FONTE.

Ficha catalográfica preparada pela Seção de Atendimentos ao Usuário do Serviço de Biblioteca - EESC/USP.

G333a Gennaro, Elmer Mateus
G3álise da estabilidade global de escoamentos compressíveis. / Elmer Mateus Gennaro; orientador Marcello Augusto Faraco de Medeiros; coorientador Vassilios Theofilis. São Carlos, 2012.

Tese (Doutorado) - Programa de Pós-Graduação em Engenharia Mecânica e Área de Concentração em Aeronaves -- Escola de Engenharia de São Carlos da Universidade de São Paulo, 2012.

1. Instabilidade hidrodinâmica. 2. Análise estabilidade linear Biglobal. 3. Problema de autovalor. 4. Método de Arnoldi. I. Título. 


\section{FOLHA DE JULGAMENTO}

Candidato: Licenciado ELMER MATEUS GENNARO.

Título da tese: "Análise da estabilidade global de escoamentos compressíveis".

Data da defesa: 08/08/2012

Comissão Julgadora:

Resultado:

Prof. Associado Marcello Augusto Faraco de Medeiros (Orientador) Aprovado (Escola de Engenharia de São Carlos/EESC)

Prof. Dr. Carlos De Marqui Junior

(Escola de Engenharia de São Carlos/EESC)

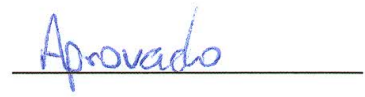

Prof. Associado Oscar Mauricio Hernandez Rodriguez

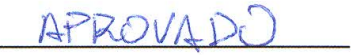

(Escola de Engenharia de São Carlos/EESC)

Prof. Dr. Sávio Brochini Rodrigues

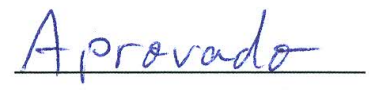

(Universidade Federal de São Carlos/UFRJ)

Prof. Titular Julio Romano Meneghini

(Escola Politécnica/USP)

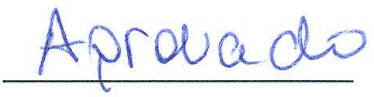

Coordenador do Programa de Pós-Graduação em Engenheira Mecânica:

Prof. Associado Marcelo Areias Trindade

Presidente da Comissão de Pós-Graduação:

Prof. Titular Denis Vinicius Coury 

Ao meus pais, e à minha esposa, com Amor e Gratidão. 



\section{Agradecimentos}

A Deus, por conceder-me bençãos imensuráveis além de encaminhar oportunidades e principalmente as pessoas que me apoiaram e me incentivaram.

À minha esposa, Lisa, pelo amor, companheirismo e incentivo nos momentos de dificuldades.

Ao meus pais, meus irmãos, sobrinhos e família pelo carinho, cuidado, compreensão, ensinamentos e o amor desprendido sem reservas.

Ao Professor Marcello Augusto Faraco de Medeiros a ao Prof. Vassilios Theofilis, pelos incentivos e orientações essenciais e necessárias para a realização deste trabalho.

Ao amigo Daniel Rodríguez-Álvarez, pelas orientações e valorosas discussõses que foram essenciais para a realização deste trabalho.

Aos meus co-autores, aos meu amigo José Miguel Pérez-Pérez, aos alunos da pós-aero, pela amizade e o apoio neste desafio.

À Escola de Engenharia de São Carlos e Universidad Politécnica de Madrid, pela infraestrutura concedida para a realização deste trabalho.

Ao suporte de Marie Curie (Grant PIRSES-GA-2009-247651 "FP7-PEOPLE-IRSES" : ICOMASEF - Instability and COntrol of MAssively SEparated Flows).

À CAPES, pelo apoio financeiro concedido na forma de bolsa de estudo para estágio de doutorado.

Ao CNPq, pelo apoio financeiro concedido na forma de bolsa de estudo. 


\section{Sumário}

Lista de Figuras

Lista de Tabelas

XV

Resumo

xvii

Abstract

xix

1 Introdução

2 Teoria

2.1 Equações Governantes . . . . . . . . . . . . . . . . . . . 7

2.2 Teoria de estabilidade linear . . . . . . . . . . . . . . . . . . . . . . . 9

2.2.1 Teoria de estabilidade linear - Local . . . . . . . . . . . . . . . 10

2.2 .2 Teoria fracamente não linear . . . . . . . . . . . . . . . . . . . 12

2.2.3 Teoria de estabilidade linear - Não-Local . . . . . . . . . . . . . . . 13

2.2.4 Teoria de estabilidade linear - Global . . . . . . . . . . . . . 13

3 Metodologia Numérica $\quad 17$

3.1 Método de colocação espectral . . . . . . . . . . . . . . . . 17

3.2 Diferenças finitas com erro uniforme . . . . . . . . . . . . . 20

3.3 Produto de Kronecker . . . . . . . . . . . . . . . . . . . . . . 21

3.4 Operador diferencial . . . . . . . . . . . . . . . . 22

3.5 Mapeamento da Malha Computacional . . . . . . . . . . . . . 25 
3.6 Método de Arnoldi . . . . . . . . . . . . . . . . . . . . . 26

3.7 Solução do Problema de Autovalor por Matriz Esparsa . . . . . . . . . . . 29

4 Casos para verificação e desenvolvimento do código 35

4.1 Problema de Autovalor de Laplace . . . . . . . . . . . . . . . . . . . . 35

4.2 Duto Retangular . . . . . . . . . . . . . . . . . . . . . 38

4.3 Instabilidade linear de um escoamento sobre uma cavidade com tampa

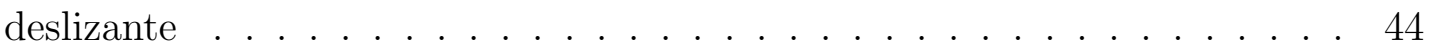

5 Camada limite de uma linha de estagnação de uma asa 49

5.1 Escoamento Base . . . . . . . . . . . . . . . . . . . . . 52

5.2 Análise do Desempenho do Algorítmo Implementado . . . . . . . . . . . . 55

5.3 Resultados da análise de estabilidade BiGlobal . . . . . . . . . . . . . . 73

6 Considerações finais e trabalhos futuros $\quad 91$

$\begin{array}{lll}7 & \text { Apêndice } & 95\end{array}$

7.1 Operador compressível linearizado . . . . . . . . . . . . . . . 95

7.2 Descrição método de Arnoldi . . . . . . . . . . . . . . . . . . . . . 99

7.3 Dados do escoamento base de uma camada limite compressível ao longo do bordo de ataque de uma asa enflechada . . . . . . . . . . . . . . . . 101

7.4 Formato da matriz esparsa comprimida . . . . . . . . . . . . . . . 102 


\section{Lista de Figuras}

2.1 Taxa de amplificação temporal, referente ao modo simétrico, como função de $\alpha$ para um escoamento de Poiseulli para $R e=10^{4}$. . . . . . . . . . . . 12

3.1 Malha computacional discretizada em pontos de Chebyshev-Gauss-Lobatto. 19

3.2 Domínio computacional discretizado em $N_{x}=N_{y}=21$ pontos usando métodos das diferenças finitas com erro uniforme. . . . . . . . . . . . . . 21

3.3 Estrutura da matriz diferenciação para um domínio computacional $N_{x}=$

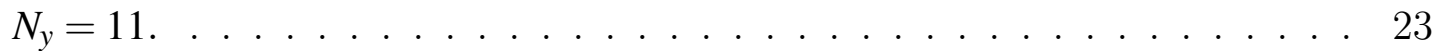

3.4 Estrutura da matriz diferenciação para um domínio computacional $N_{x}=$ $N_{y}=21 \ldots \ldots \ldots \ldots \ldots \ldots \ldots \ldots \ldots$

3.5 Autovalores obtidos para o escoamento em uma cavidade. Símbolos "×"representam os autovalores obtidos pelo algorítmo de Arnoldi e os símbolos "o" são os autovalores obtidos pelo método QZ . . . . . . . . . . . . . . . . 29

3.6 Padrão de esparsidade do operador $\mathscr{L}$ referente aos problemas de Poisson, escoamento incompressível e compressível, discretizados em $N x=N y=13$. Para efeitos de comparação, as figuras foram geradas fixando a dimensão da matriz correspondente ao caso compressível.

3.7 Padrão de esparsidade do operador $\mathscr{R}$ referente aos problemas de Poisson, escoamento incompressível e compressível, discretizados em $N x=N y=13$ para ambos métodos.

4.1 Autovetores correspondentes aos 4 primeiros modos associados ao problema de Laplace bi-dimensional homogêneo.

4.2 Erro numérico associado ao cálculo do modo 34 que corresponde à $i=5$ e $j=3$ na solução (4.3) em distintos métodos e ordem de discretização espacial: diferenças finitas padrão (STD) e com erro uniforme (DFq) e pontos de Chebyshev-Gauss-Lobatto. 
4.3 Autovetores correspondentes aos 4 primeiros modos associados ao problema de Laplace bi-dimensional em que $f(x, y)=e^{(20(y-x-1))} \ldots \ldots . . . . .37$

4.4 Esquema geométrico de um duto retangular. . . . . . . . . . . . . . . 38

4.5 Erro de truncamento da solução analítica com respeito ao valor convergido $(A=1 \rightarrow n=8, A=2 \rightarrow n=4) \ldots \ldots \ldots$

4.6 Histórico de convergência da solução numérica da equação de Poisson para razão de aspecto unitário $(A=1)$. O valor de $n=8$ foi assumido na Eq. (4.7), proposta por Rosenhead (1963), como valor referência. . . . . . . . . 41

4.7 Autofunções, $u, v, w$, correspondente ao primeiro modo de um escoamento em um duto retangular, de razão de aspecto unitário. . . . . . . . . . . . 41

4.8 Memória computacional empregada na decomposição LU de um problema de autovalor para um escoamento em duto retangular de razão de aspecto unitário, $R e=100$ e $\beta=1 . \quad \ldots \ldots \ldots$. . . . . . . . . . . . . 42

4.9 Tempo computacional empregado na decomposição LU de um problema de autovalor para um escoamento em duto retangular de razão de aspecto

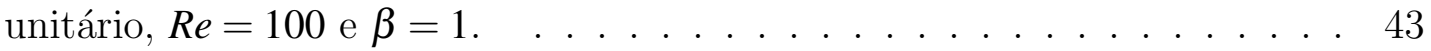

4.10 Erro relativo para o modo menos estável de um escoamento em um duto retangular de razão de aspecto unitário, $R e=100$ e $\beta=1 . \quad \ldots . . .43$

4.11 Linhas de corrente de uma cavidade fechada para $R e=2000$. . . . . . . . 44

4.12 Componentes de velocidade de uma cavidade fechada regularizada para $R e=582$.

4.13 Espectro completo obtido para o problema da cavidade fechada $R e=782$ e $\beta=15.3 \ldots \ldots \ldots \ldots \ldots$. . . . . . . . . . . . . . . . . . . . . .

5.1 Representação esquemática dos vetores velocidade do escoamento externo. $\quad 50$

5.2 Representação esquemática 2D dos vetores velocidade do escoamento. . . . 51

5.3 Solução do escoamento base. (a) componentes de velocidade longitudinal (linhas sólidas) e transversal (linhas tracejadas) para números de Mach $M a=0.02$ (cor preta) e $M a=0.90$ (cor vermelha). (b) perfis de temperatura para diferentes números de Mach. . . . . . . . . . . . . . 55

5.4 Linhas de corrente sobrepostas ao campo de velocidade $u$ em $M a=0.90 \quad$. 56 
5.5 Porcetagem dos elementos não-nulos em relação aos elementos da matriz original dado por $\left(n_{x} \times n_{y} \times n_{\mathrm{v} a r}\right)^{2}$, onde $n_{\mathrm{v} a r}=4$ e $n_{\mathrm{v} a r}=5$ correspondem ao escoamento incompressível e compressível, respectivamente. . . . . . . . 61

5.6 Coeficiente de preenchimento em função do método de ordenação e discretização em escala semi-logarítmica. . . . . . . . . . . . . . . .

5.7 Estimativa da memória escalonada pela memória requerida no caso denso para os diferentes métodos de ordenação e discretização espacial. . . . . . . .

5.8 Tempo de processamento (CPU) escalado com a memória requerida na solução densa $\left(N^{3}\right)$, para diferentes resoluções, métodos e discretizações.

5.9 Distribuição do erro relativo referente ao modo GH para $M a=0.9, R e=800$ e $\beta=0.19$ correspondente a cada distretização espacial. . . . . . . . . . . . 72

5.10 Parte real da autofunção em $M a=0.9, R e=800$ e $\beta=0.19$ correspondente aos modos GH ( $1^{a}$ linha), A1 ( $2^{a}$ linha), S2 ( $3^{a}$ linha) e A2 (4 ${ }^{a}$ linha). Coluna esquerda: $u$-velocidade, Coluna direita: $z$-velocidade.

5.11 Dependência de $c_{i}$ em relação a $\beta$ para o modo GH em (a) $M a=0.5,0.02$ e $R e=800$ (b) $M a=0.9, R e=800$ e $R e=1500$. Linhas sólidas foram obtidas da análise assintótica e símbolos quadrados pela análise BiGlobal de (THEOFILIS; FEDOROV; COLLIS, 2004). Símbolos círculos são resultados do presente trabalho. . . . . . . . . . . . . . . .

5.12 Taxa de amplificação temporal como função de $\beta$ em (a) $\operatorname{Re}=1500$, (b) $R e=800$ e (c) $R e=585 \ldots \ldots \ldots \ldots \ldots$. . . . . . . . . . . . . . . . . . . . .

5.13 Dependência da taxa de amplificação temporal (a)-(b) e frequência (c)-(d) dos modos GH, A1 e S2 com $\beta$ para $M a=0.50$ e $M a=0.90$. . . . . . . .

5.14 Distribuição de $\omega_{i}$ calculado em $R e=1500$ para uma ampla banda de número de onda $\beta$ e uma faixa de número de Mach de $0.02-1.90$. . . . . . 86

5.15 Taxa de amplificação temporal correspondente à uma faixa de número de Mach, $R e=1500$ e número de onda fixado. . . . . . . . . . . . . . 86

5.16 Taxa de amplificação temporal $\omega_{i}$ correspondente ao número de onda $\beta$ que mais amplifica como função de número de Reynolds. As linhas correspondem a $M a=\{0.02,0.2,(0.1), 0.9\}$ na direção da seta. . . . . . . . . . 
5.17 Número de onda correspondente a máxima taxa de amplificação temporal versus número de Reynolds. . . . . . . . . . . . . . . . . . . . . . . 87

5.18 Perfis normal à parede para velocidade $w$ (direta) e temperatura (esquerda) do modo dominante $\mathrm{GH}$, como função de $M a=0.9, R e=800$ e $\beta=0.19 . \quad$. 88

5.19 Curvas de estabilidade neutra para uma camada limite compressível de uma linha de estagnação de uma asa enflechada obtidas através da análise BiGlobal. Símbolos representam resultados anteriores apresentados por (THEOFILIS; FEDOROV; COLLIS, 2004). . . . . . . . . . . . . . . 89

5.20 Número de onda $\beta$ crítico correspondente a cada número de Mach calculado dentro da faixa subsônica. . . . . . . . . . . . . . . . . . . . . . . . 89

5.21 Valores extraídos das curvas neutras nas condições críticas para números de Reynolds em diferentes números de Mach. . . . . . . . . . . . . . . . . . 90 


\section{Lista de Tabelas}

2.1 Decomposições da teoria de estabilidade linear . . . . . . . . . . . . . . . . 10

3.1 Estimativa elementos não-nulos . . . . . . . . . . . . . . . . . . 25

4.1 Comparação numérica do problema bi-dimensional de autovalor de Laplace discretizado em 12 pontos de Chebyshev-Gauss-Lobatto. . . . . . . . . . . 36

4.2 Comparação numérica dos autovalores obtidos pelo presente trabalho. O problema foi discretizado em 24 pontos CGL. . . . . . . . . . . . . . . 36

5.1 Dependência de $U_{0}{ }^{\prime}(0), W_{0}{ }^{\prime}(0)$ e temperatura na parede do escoamento base em vários Ma . . . . . . . . . . . . . . . . . . . . . . . . . . . . . . . . . . . 54

5.2 Step I: Resultado dos coeficientes de preenchimento como função da resolução da malha computacional, para solução numérica do problema de autovalor BiGlobal discretizado em pontos de colocação espectral em ambas direções, usando diferentes métodos de ordenação . . . . . . . . . . . . . . 58

5.3 Step I: Resultado dos coeficientes de preenchimento como função da resolução da malha computacional e distretização espacial em diferenças finitas em ambas direções, para solução numérica do problema de autovalor BiGlobal do escoamento compressível, usando diferentes métodos de ordenação . . . . . . . . . . . . . . . . . . . . . . .

5.4 Step I: Step I: Resultado dos coeficientes de preenchimento como função da resolução da malha computacional e distretização espacial em diferenças finitas na direção na direção $x$ e Chebyshev-Gauss-Lobatto $y$, e vice-versa para solução numérica do problema de autovalor BiGlobal do escoamento compressível, usando diferentes métodos de ordenação. . . . . . .

5.5 Step II: Fatoração simbólica. Estimativa da memória (Mb) requirida para a decomposição $L U$ para escoamento incompressível e diferentes métodos de ordenação. . . . . . . . . . . . . . . . . . . . . . 66 
5.6 Step II: Fatoração simbólica. Estimativa da memória requirida para a decomposição $L U(\mathrm{Mb})$ para escoamento compressível e diferentes métodos de ordenação e discretização. . . . . . . . . . . . . . . . . . . . . . 67

5.7 Step III: $C P U$-time para fatorização (s) para o caso imcompressível. . . . . 68

5.8 Step III: $C P U$-time para fatorização (s) para o caso compressível. . . . . . . 69

5.9 Memória e tempo de CPU para a solução do problema de autovalor BiGlobal para a convergência do modo dominante acima de 6 casas decimais, para uma combinação de métodos de discretização. O problema físico testado é o escoamento ao longo do bordo de ataque de uma asa enflechada em $R e=800, M a=0.9$ e $\beta=0.19$. . . . . . . . . . . . . . . . . 73

5.10 Histórico do refinamento da malha computacional da solução numérica da camada limite de uma linha de estagnação incompressível $(M a=0.02)$ de um bordo de ataque de uma asa em $\operatorname{Re}=800, \beta=0.255$ e $x \in[-100,+100] .74$

5.11 Estudo de independência do domínio computacional em $R e=800, M a=$ 0.90 e $\beta=0.19$ para uma malha computacional $140 \times 140$ (DF6-CGL). Domínio abaixo de $[-100,+100]$ fornece resultados pobres para modo S2. . 75

5.12 Estudo de refinamento de malha computacional na solução numérica para a camada limite de uma linha de estagnação compressível no domínio $[-100,100]$ para $R e=800, M a=0.90$ e $\beta=0.19$ para o cálculo dos 3 primeiros modos $\mathrm{GH}, \mathrm{A} 1 \mathrm{e} \mathrm{S} 2 \ldots \ldots \ldots . \ldots . \ldots 76$

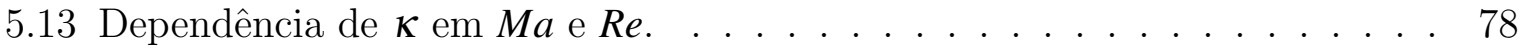

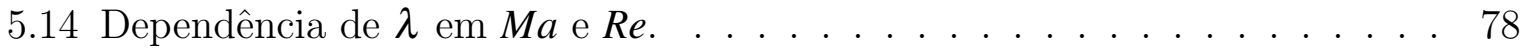

5.15 Valores críticos $\left(R e_{\text {crit }}\right.$ e $\left.\beta_{\text {crit }}\right)$ correspondente vários números de Mach. . . 81

7.1 Dependência de $U_{0}{ }^{\prime}(0), W_{0}{ }^{\prime}(0)$ e temperatura na parede do escoamento base para $M a \in\left[10^{-5}, 5.00\right] \ldots \ldots \ldots 10 . \ldots \ldots$

7.2 Exemplo da matriz $M$ no formato CSR . . . . . . . . . . . . . . 102 


\section{Resumo}

Gennaro, E.M. (2012). Análise da Estabilidade Global de Escoamentos Compressíveis. São Carlos, 2012. - Escola de Engenharia de São Carlos, Universidade de São Paulo.

A investigação dos mecanismos de instabilidade pode ter um papel importante no entendimento do processo laminar para turbulento de um escoamento. Análise de instabilidade de uma camada limite de uma linha de estagnação compressível foi realizada no contexto de teoria linear BiGlobal. O estudo dos mecanismos de instabilidade deste escoamento pode proporcionar uma visão útil no desenho aerodinâmico das asas. Um novo procedimento foi desenvolvido e implementado computacionalmente de maneira sequencial e paralela para o estudo de instabilidade BiGlobal. O mesmo baseia-se em formar a matriz esparsa associada ao problema discretizado por dois métodos: pontos de colocação de Chebyshev-Gauss-Lobatto e diferenças finitas, além da combinações destes métodos. Isto permitiu o uso de bibliotecas computacionais eficientes para resolver o sistema linear associado ao problema de autovalor utilizando o algoritmo de Arnoldi. O desempenho do método numérico e código computacional proposto são analisados do ponto de vista do uso de métodos de ordenação dos elementos da matriz, coeficientes de preenchimento, memória e tempo computacional a fim de determinar a solução mais eficiente para um problema físico geral com técnicas de matrizes esparsas. Um estudo paramétrico da instabilidade da camada limite de uma linha de estagnação foi realizado incluindo o estudo dos efeitos de compressibilidade. O excelente desempenho código computacional permitiu obter as curvas neutras e seus respectivos valores críticos para a faixa de número de Mach $0 \leq M a \leq 1$. Os resultados confirmam a teoria assintótica apresentada por (THEOFILIS; FEDOROV; COLLIS, 2004) e mostram que o incremento do número de Mach reduz o número de Reynolds crítico e a faixa instável do número de ondas.

Palavras chave: Instabilidade hidrodinâmica, Análise estabilidade linear BiGlobal, Problema de autovalor, Método de Arnoldi, Escoamento Compressível. 


\section{Abstract}

Gennaro, E.M. (2012). Global instability analysis of compressible flow. São Carlos, 2012. - Escola de Engenharia de São Carlos, Universidade de São Paulo.

Investigation of linear instability mechanisms is essential for understanding the process of transition from laminar to turbulent flow. An algorithm for the numerical solution of the compressible BiGlobal eigenvalue problem is developed. This algorithm exploits the sparsity of the matrices resulting from the spatial discretization of the enigenvalue problem in order to improve the performance in terms of both memory and CPU time over previous dense algebra solutions. Spectral collocation and finite difference spatial discretization methods are implemented, and a performance study is carried out in order to determine the best practice for the efficient solution of a general physical problem with sparse matrix techniques. A combination of spectral collocation and finite differences can further improve the performance. The code developed is then applied in order to revisit and complete the parametric analyses on global instability of the compressible swept Hiemenz flow initiated in (THEOFILIS; FEDOROV; COLLIS, 2004) and obtain neutral curves of this flow as a function of the Mach number in the $0 \leq M a \leq 1$ range. The present numerical results fully confirm the asymptotic theory results presented in (THEOFILIS; FEDOROV; COLLIS, 2004). This work presents a complete parametric study of the instability properties of modal three dimensional disturbances in the subsonic range for the flow configuration at hand. Up to the subsonic maximum Mach number value studied, it is found that an increase in this parameter reduces the critical Reynolds number and the range of the unstable spanwise wavenumbers.

Keywords: Hydrodynamic instability, BiGlobal instability analysis, Eigenvalue problem, Arnoldi Method, Compressible flow. 


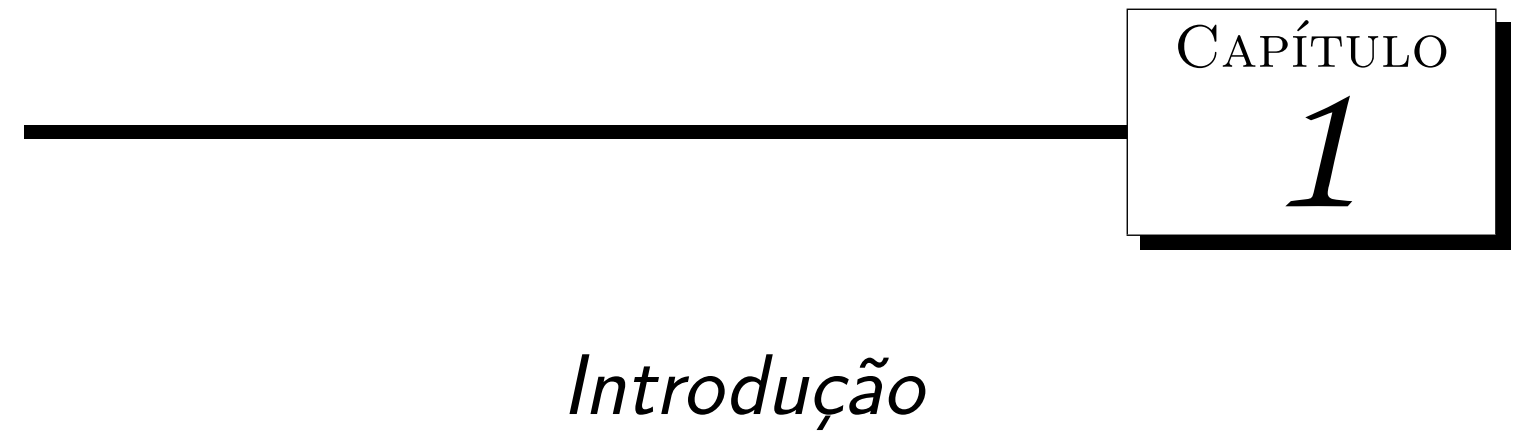

A investigação dos mecanismos de instabilidade pode ter um papel importante no entendimento do processo laminar para turbulento de um escoamento. De fato, a instabilidade hidrodinâmica explica o processo pelo qual um escoamento em regime permanente espontaneamente se torna transiente. É a partir de uma instabilidade hidrodinâmica que o escoamento se torna turbulento (MEDEIROS, 2000) ou emite som (COLONIOUS; LELE, 2004). Por várias décadas, muitos estudos essencialmente uni-dimensionais têm sido apresentados, do ponto de vista de escoamento base, tais como camadas limites e camadas de mistura. A principal razão disso é que a geometria e o estado básico em muitas aplicações dependem de formas não-homogêneas em mais de uma direção espacial, o que não permite o uso de equações simplificadas. Ao considerar duas ou três direções não-homogêneas na formulação do problema de estabilidade, a solução resulta em um formidável custo computacional para um estudo paramétrico completo, que são, por outro lado, mandatório do ponto de vista da física do problema.

Se em alguma situação um escoamento base, laminar, uniforme ou não uniforme, pode ser estabelecido, as equações de Navier-Stokes podem ser escritas em termos das variáveis de perturbação e linearizadas sobre o escoamento base. Essa linearização é importante para estudar o desenvolvimento de pequenas perturbações neste escoamento, isto é, a resposta do escoamento a essa perturbações. Se o escoamento depende de duas dire- 
ções espaciais não-homogêneas, a perturbação pode ser considerada periódica ao longo da terceira direção espacial homogênea e então introduz-se os modos de Fourier. Uma dependência exponencial temporal é assumida para as perturbações e consequentemente tem-se um problema de autovalor generalizado bi-dimensional, onde os autovalores são frequências complexas e as autofunções dependem das duas direções espaciais não-homogêneas. Esta abordagem é conhecida como análise de instabilidade linear BiGlobal (THEOFILIS, 2003; THEOFILIS, 2011) mas poderia ser chamada de "Análise da instabilidade linear Global". No entanto, essa diferenciação é feita a fim de evitar confusão com o uso desse termo por Chomaz, Huerre e Redekopp (1988). Em princípio, todas essas hipóteses levam à um problema mais fácil de resolver que fazer simulações numéricas diretas (DNS), embora o tamanho da matriz discretizada faz da solução numérica um grande desafio.

Theofilis (2011) apresenta uma análise da instabilidade linear BiGlobal como um caminho na qual se obtém informações úteis dos escoamentos sobre ou através de geometrias complexas, além de ser um caminho promissor na elaboração de estratégias de controle de escoamento teoricamente fundamentada. Escoamentos complexos se desenvolvem em extensos domínios e se comportam como amplificadores de ruído ou osciladores exibindo fortes não - linearidades. Deste modo, a dinâmica do escoamento pode ser vista como um resultado da interação entre modos globais que ocupam todo o domínio físico. Uma investigação destes modos globais tem sido progressivamente mais utilizada em vista do aumento da capacidade computacional (HUERRE; MONKEWITZ, 1990; THEOFILIS, 2011) ou do aprimoramento das metodologias.

As soluções para o problema de autovalor generalizado associado à análise de instabilidade linear BiGlobal, essencialmente possuem duas abordagens. As duas abordagens diz respeito à construção ou não do operador linear. Ambas tem vantagens e desvantagens, mas em princípio destaca-se somente a quantidade de autovalores que se obtém a partir da abordagem que baseia-se na construção do operador linear em oposição aos métodos de matriz-livre. No escopo de uma abordagem que se baseia na construção do operador linear, o fato do tamanho da matriz discretizada associada ao problema de autovalor ser 
grande, a solução do problema de autovalor completo torna-se um grande desafio. Dessa forma, é imprescindível utilizar técnicas mais apuradas e eficientes, do ponto de vista computacional, para resolver o problema de autovalor e o entendimento da física intrínsica ao problema. Uma das técnicas mais eficientes e úteis para resolver um problema de autovalor generalizado está baseada nos métodos de projeção iterativa, tal como o método de Arnoldi que está baseado no subespaço de Krylov (SAAD, 2000). Através desse método obtém-se uma porção dos autovalores do problema completo, ou ainda, uma janela do autoespectro completo. A desvantagem desse método é que o mesmo prioriza os autovalores de maior módulo. Em geral, para um problema de estabilidade linear estamos interessados nos autovalores que estão próximos aos eixos e origem do plano complexo. Por esta razão, para o emprego deste método tem-se que utilizar transformações analíticas de modo a calcular a região de interesse do autoespectro, por exemplo, transformações que fazem um "shift-invert" nos autovalores.

Uma solução do problema de estabilidade BiGlobal dentro do contexto de formação de matriz foi apresentado por Rodríguez e Theofilis (2009) e Kitsios et al. (2009). O uso de algoritmos de álgebra linear densa nestes trabalhos apontam para a necessidade do uso de estratégias de armazenagem da matriz bem como em metodologias de inversões, especialmente para os casos em que o escoamento é compressível. O custo computacional para realizar uma decomposição das matriz em LU ${ }^{1}$ dos operadores discretizados em resoluções típicas para recuperar a física usando álgebra linear densa facilmente torna-se proibitivo, e assim o desenvolvimento de uma metodologia alternativa torna-se obrigatório. Uma alternativa é substituir a forma de armazenar a matriz e as operações por métodos de álgebra esparsa. Hernández et al. (2007) apresenta uma visão geral de métodos esparsos para problemas de autovalores. Problemas modelos ou acadêmicos, em geral, possuem propriedades especiais, por exemplo, matrizes simétricas definida positiva, e são frequentemente apresentados para demonstrar os benefícios dos algoritmos que exploram a esparsidade. Por outro lado, as matrizes que descrevem as equações de navier-Stokes linearizadas são altamente não-normais (SCHMID; HENNINGSON, 2001).

\footnotetext{
${ }^{1} \mathrm{LU}$ vem do inglês lower e upper
} 
Crouch, Garbaruk e Magidov (2007b) e Crouch et al. (2009) foram os primeiros a implementar técnicas de álgebra linear esparsa para a solução de um problema de instabilidade compressível BiGlobal e demonstraram que utilização do software MUMPS ${ }^{2}$ (AMESTOY et al., 2001) é uma alternativa eficiente para a paralelização massiva do problema análogo tratado como denso. Um fator decisivo foi o uso de baixa ordem para métodos de diferenças finitas, de modo que as matrizes resultantes discretizadas são substancialmente mais esparsas do que as matrizes resultantes da discretização por colocação espectral usado por Rodríguez e Theofilis (2009) e Kitsios et al. (2009). A combinação das técnicas esparsas e permutações dos métodos de colocação espectral e esquemas de diferenças finitas para a discretização espacial dos problemas compressíveis não tinha sido investigado/implementado e isso foi também uma motivação para a realização deste trabalho.

Um dos objetivos deste trabalho foi o desenvolvimento de um código computacional capaz de realizar análise de estabilidade BiGlobal de escoamentos compressíveis explorando as diversas características do problema. Do ponto de vista numérico, foi implementado e analizado métodos de decomposição LU para a matriz proveniente da discretização do problema de instabilidade linear BiGlobal. A implementação foi baseada em bibliotecas de álgebra linear como alternativa às rotinas utilizadas para abordagem densa.

Do ponto de vista físico, a presente metodologia foi aplicada a fim de estudar a instabilidade do escoamento em torno do bordo de ataque de uma asa. Este escoamento foi escolhido por duas razões. Em primeiro lugar, a partir de um ponto de vista computacional, isto é um problema bastante completo devido à presença de três componentes de velocidade do escoamento base, proporcionando uma boa avaliação dos melhoramentos promovidos pela metodologia. De fato, ao utilizar as técnicas esparsas aqui apresentadas, foi possível melhorar a precisão dos resultados apresentados anteriormente (THEOFILIS; FEDOROV; COLLIS, 2004) e ampliar a gama de parâmetros investigados. O escoamento compressível, a camada limite de uma linha de estagnação, foi analisada para demonstrar

\footnotetext{
${ }^{2}$ MUltifrontal Massively Parallel sparse direct Solver - http://graal.ens-lyon.fr/MUMPS
} 
que o aumento do desempenho pode ser substancialmente dependente do problema; particularidades na geometria do problema pode ser explorada para conceber discretizações espaciais que melhoram o desempenho. Neste problema, os requisitos de resolução em uma direção espacial são menos rigorosas do que na outra, e uma mistura de métodos de colocação espectral - diferenças finitas para discretização espacial foi a solução ideal encontrada. Em segundo lugar, a partir de um ponto de vista físico, a instabilidade do escoamento próximo do bordo de ataque das asas enflechadas tem um significado de engenharia. Este tipo de instabilidade promove as fases iniciais do crescimento de perturbações que serão convectadas à jusante, tendo influência direta no processo geral de transição de laminar para escoamento turbulento na superfície da asa, e por sua vez, afetando o desempenho aerodinâmico. O estudo dos mecanismos de instabilidade deste escoamento pode proporcionar uma visão útil no desenho aerodinâmico das asas.

Ao contrário da maioria dos trabalhos citados anteriormente, no qual a computação em grande escala foi utilizada para estabelecer o escoamento base e estudar o desenvolvimento instabilidade subsequente, a presente contribuição faz uso da abordagen computacional eficiente recém desenvolvida, revisitando os resultados apresentados em (THEOFILIS; FEDOROV; COLLIS, 2004), e completando em um computador pessoal o estudo paramétrico iniciado e melhorando a precisão.

O trabalho está divido em capítulos, seções e subseções. O capítulo 2 apresenta a fundamentação teórica do trabalho e as equações utilizadas, enquanto o capítulo 3 apresenta alguns detalhes da formulação do problema e da solução numérica do problema de autovalor. Capítulo 4 apresenta testes e uma pequena discussão sobre o desempenho está no capítulo 5, onde pode ser visto estimativas de memória e requisitos de tempo de CPU para diferentes combinações de métodos de discretização. Posteriormente a implementação os resultados são verificados para os casos incompressível e compressível. Os ganhos de eficiência oferecidos pelo algoritmo de cálculo proposto permitiu pela primeira vez calcular a curva neutra para o caso compressível ortogonal. Estes resultados são apresentados no capítulo 5. Finalmente, algumas considerações são apresentadas no capítulo 6 . 


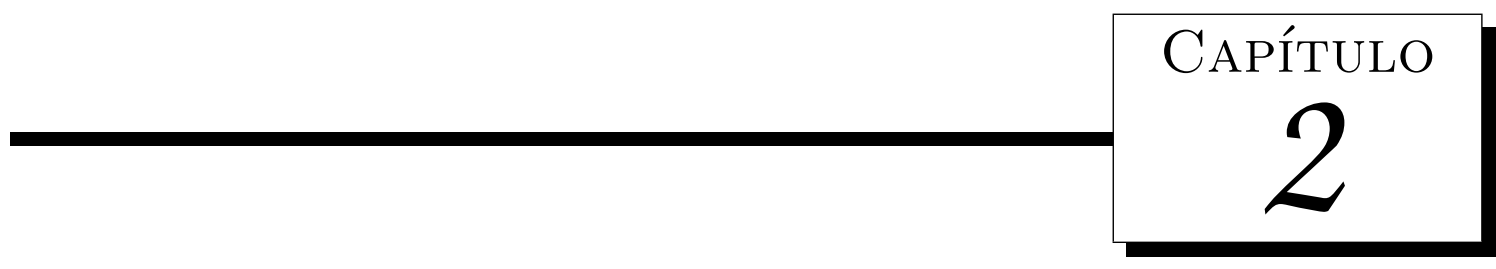

\section{Teoria}

\subsection{Equações Governantes}

As equações governantes para um escoamento incompressível são escritas a seguir na formulação variável primitiva

$$
\begin{aligned}
\frac{\partial u_{i}}{\partial x_{i}} & =0 \\
\frac{\partial u_{i}}{\partial t}+u_{j} \frac{\partial u_{i}}{\partial x_{j}} & =-\frac{\partial p}{\partial x_{i}}+\frac{1}{\operatorname{Re}} \frac{\partial^{2} u_{i}}{\partial x_{j}^{2}}
\end{aligned}
$$

As equações governantes para um escoamento compressível são descritas a seguir.

Equação da continuidade

$$
\frac{\partial \rho}{\partial t}+\frac{\partial \rho u_{j}}{\partial x_{j}}=0
$$

Equação de quantidade de movimento definida para cada componente de velocidade

$$
\frac{\partial \rho u_{i}}{\partial t}+\frac{\partial \rho u_{i} u_{j}}{\partial x_{j}}=-\frac{\partial p}{\partial x_{i}}+\frac{\partial \tau_{i j}}{\partial x_{j}}
$$

Equação da energia é dada por:

$$
\frac{\partial E_{T}}{\partial t}+\frac{\partial u_{j}\left(E_{T}+p\right)}{\partial x_{j}}=-\frac{\partial q_{j}}{\partial x_{j}}+\frac{\partial u_{j} \tau_{i j}}{\partial x_{j}}
$$

Nas equações acima, $x_{i}, x_{j}(i, j=1,2,3)$ representam as coordenadas espaciais longitu- 
dinal, normal e transversal ao escoamento e serão denotadas neste trabalho por $x, y$ e $z$, respectivamente, $t$ o tempo, $u_{i}, u_{j}$ as componentes de velocidade $(u, v, w), \rho$ a densidade e $p$ a pressão.

A energia total é dado por

$$
E_{T}=\rho\left(e+\frac{u^{2}+v^{2}+w^{2}}{2}\right)
$$

e a variável primitiva $e$ é a energia interna.

As relações constitutivas adimensionais para um fluido Newtoniano e condução de calor de Fourier são

$$
\begin{aligned}
\tau_{i j} & =\frac{1}{\operatorname{Re}}\left(\frac{\partial u_{i}}{\partial x_{j}}+\frac{\partial u_{j}}{\partial x_{i}}-\frac{2}{3} \frac{\partial u_{k}}{\partial x_{k}} \delta_{i j}\right) \\
q_{j} & =-\frac{1}{(\gamma-1) M a^{2} \operatorname{PrRe} e} \frac{\partial T}{\partial x_{j}},
\end{aligned}
$$

onde o número de Prandtl é definido como $P r=\frac{c_{p} \mu}{k}, \gamma$ é a razão de calor específico, $c_{p}$ o calor específico para pressão constante, $k$ é a condutividade térmica, número de Reynolds e o número de Mach são definidos como

$$
R e=U_{r e f} L_{r e f} / v, \quad M a=U_{r e f} / \mathrm{a}
$$

onde a é a velocidade do som e $v$ a viscosidade cinemática.

A lei dos gases perfeitos para pressão e temperatura adimensionais são dadas por:

$$
p=(\gamma-1) \rho e
$$

$\mathrm{Ou}$

$$
T=\frac{\gamma M a^{2} p}{\rho}
$$

Para a viscosidade, foi empregada a fórmula de Sutherland

$$
\mu=T^{3 / 2} \frac{1+C}{T+C}
$$

onde usualmente $C=101.4 / 300$. 


\section{$2.2 \quad$ Teoria de estabilidade linear}

Uma análise da estabilidade hidrodinâmica linear é realizada geralmente através do método da decomposição em modos normais (DRAZIN; REID, 1981; CRIMINALE; JACKSON; JOSLIN, 2003; MENDONÇA, 2003), a semelhança do que se faz para sistemas mecânicos sólidos. A teoria consiste basicamente em investigar a resposta do escoamento a perturbações, com devido interesse nas perturbações infinitesimais. É uma técnica ${ }^{1}$ já estabelecida, utilizada por muitos autores e com resultados comprovados tanto por experimentos como por simulações computacionais em uma vasta gama de escoamentos, incluindo os regimes subsônico, transônico e supersônico, escoamentos monofásicos, ou ainda bi-fásicos, na presença de superfícies livre e paredes ou não, etc.

Na teoria linear de instabilidade hidrodinâmica clássica o escoamento é decomposto em uma parte permanente, chamada escoamento base ${ }^{2}$, e uma parte flutuante, a perturbação. O escoamento base é um dado do problema, a perturbação é a incógnita. Assumindo-se que a perturbação é infinitesimal, as equações de movimento para a perturbação podem ser linearizadas. Com esta hipótese as equações de movimento para a perturbação se tornam separáveis, propriedade essencial para o desenvolvimento dessa teoria.

A tabela 2.1 apresenta resumidamente uma classificação dos diferentes tipos de teoria de estabilidade linear empregadas. Essa classificação é baseada nas hipóteses adotadas para o escoamento base. São demarcadas as fronteiras entre análise local, baseada em variações da equação de Orr-Sommerfeld (OSE), análise não-local, baseadas nas equações parabolizadas de estabilidade (PSE) e as três versões da teoria de análise global.

A seguir, apresentaremos um detalhamento das teorias indicadas na tabela 2.1 a fim de contextualizar a teoria de estabilibilidade linear Global utilizada nesse trabalho.

\footnotetext{
1 Também utilizada na verificação de códigos computacionais pela comparação de soluções

${ }^{2}$ Independente do tempo
} 
Tabela 2.1: Decomposições da teoria de estabilidade linear

\begin{tabular}{l|l|l|cl}
\hline & Teoria & Esc. Base & \multicolumn{2}{|c}{ Modos } \\
& & & Função amplitude & Função fase \\
\hline \multirow{3}{*}{ Global } & TriGlobal & $\overline{\mathbf{q}}(x, y, z)$ & $\hat{\mathbf{q}}(x, y, z)$ & $-\omega t$ \\
& PSE-3D & $\overline{\mathbf{q}}\left(x^{*}, y, z\right)$ & $\hat{\mathbf{q}}\left(x^{*}, y, z\right)$ & $\int \alpha\left(x^{*}\right) d x^{*}-\omega t$ \\
& BiGlobal & $\overline{\mathbf{q}}(x, y)$ & $\hat{\mathbf{q}}(x, y)$ & $\beta z-\omega t$ \\
\hline Não-local & PSE & $\overline{\mathbf{q}}\left(x^{*}, y\right)$ & $\hat{\mathbf{q}}\left(x^{*}, y\right)$ & $\int \alpha\left(x^{*}\right) d x^{*}+\beta z-\omega t$ \\
\hline Local & OSE & $\overline{\mathbf{q}}(y)$ & $\hat{\mathbf{q}}(y)$ & $\alpha x+\beta z-\omega t$ \\
\hline
\end{tabular}

\subsubsection{Teoria de estabilidade linear - Local}

A teoria local é delineada quando a hipótese de escoamento base paralelo pode ser adotada, ou seja $\overline{\mathbf{q}}=(\bar{u}(y), 0,0)$, onde $\overline{\mathbf{q}}$ é o vetor velocidade base, ru é a componente longitudinal e y é perpendicular ao escoamento. Com esta hipótese as equações de movimento para a perturbação se tornam separáveis. Os coeficientes são constantes nas direções longitudinal e transversal e no tempo. Portanto, pode-se aplicar a transformada de Fourier nas direções $x$ e $z$ e a transformada de Laplace no tempo, no caso da análise ser tri-dimensional. Em outras palavras, a solução possui um caráter ondulatório nestas dimensões. Deste processo resulta um sistema de equações diferenciais ordinárias na direção y que constitui um problema de autovalor e autofunção.

As soluções do sistema são os modos normais do escoamento: o autovalor fornece a frequência e a taxa de amplificação ou atenuação exponencial do modo e a autofunção fornece o formato da onda na direção $y$. Na existência de pelo menos um modo que se amplifica, dizemos que o escoamento é instável.

Nas circunstâncias apresentadas acima, os modos normais são dados por:

$$
\mathbf{q}(x, y, t)=\underbrace{\overline{\mathbf{q}}(y)}_{\text {escoamento base }}+\varepsilon \underbrace{\hat{\mathbf{q}}(y) e^{i(\alpha x+\beta z-\omega t)}}_{\text {perturbação }}, \varepsilon \ll 1 .
$$

O número de onda (direção $x$ ) é representado por $\alpha=\alpha_{r}+i \alpha_{i}$ onde $\alpha_{r}$ é o número de onda da perturbação e $\alpha_{i}$ é a taxa de amplificação espacial. A frequência é representada por $\omega=\omega_{r}+i \omega_{i}$ onde $\omega_{r}$ é a frequência da perturbação e $\omega_{i}$ é a taxa de amplificação 
temporal.

A velocidade de grupo pode ser descrita como $c_{g}=\partial \omega / \partial \alpha$, que é a velocidade de propagação da energia do envelope de modulação.

Substituindo Eq. (2.12) nas equações de Navier-Stokes para escoamento incompressível $^{3}$, linearizando sobre o escoamento base , desprezando os termos não-lineares $\mathrm{O}\left(\varepsilon^{2}\right)$ e uma série de manipulações algébricas, obtém-se a equação conhecida por equação de Orr-Sommerfeld (ORR, 1907; SOMMERFELD, 1908) dada por

$$
\hat{v}^{\prime \prime \prime \prime}-\alpha^{2} \hat{v}^{\prime \prime}+\alpha^{4} \hat{v}-\alpha^{2} \hat{v}=i \alpha \operatorname{Re}(U-c)\left(\hat{v}^{\prime \prime}-\alpha^{2} \hat{v}\right)-i \alpha \operatorname{Re} U^{\prime \prime} \hat{v}^{\prime \prime}
$$

onde (I) representa a derivada em relação a $y$.

A análise da estabilidade é agora reduzida a um problema de autovalor do sistema de equações diferenciais em variáveis de perturbação, na qual as equações (2.12) são soluções.

Um exemplo de solução da equação de Orr-Sommerfeld (2.13), para um escoamento de Poiseulli, é apresentado na figura 2.1. Utilizou-se shooting method para obter solução e tem excelente concordância com a solução apresentada por Criminale, Jackson e Joslin (2003). Detalhes sobre o procedimento deste método pode ser encontrado em (GENNARO, 2008).

A partir da equação de Orr-Sommerfeld, a instabilidade linear pode ser descrita qualitativamente em termos físicos. Se uma perturbação é localizada no espaço, então a perturbação se propaga da mesma maneira que um pacote de onda dos modos mais instáveis, viajando com sua velocidade de grupo e crescendo exponencialmente. A perturbação decai à montante e à jusante e tranversalmente também de seu centro, a medida que viaja. Assim, para um observador se movendo com a velocidade de grupo, a perturbação cresce no tempo. Mas se a perturbação medida em uma estação fixa $x$ eventualmente decai no tempo depois que a perturbação passou pelo centro, esta instabilidade é chamada de instabilidade convectiva ${ }^{4}$. Porém, se a velocidade de grupo é zero, a instabilidade permanece

\footnotetext{
${ }^{3} \mathrm{O}$ mesmo se aplica às equações de Navier-Stokes para escoamento compressível

4 A instabilidade convectiva propaga-se ao longo do sistema, uma vez que cresce com o tempo a fim de que eventualmente a perturbação desapareça e situar-se em um determinado ponto.
} 


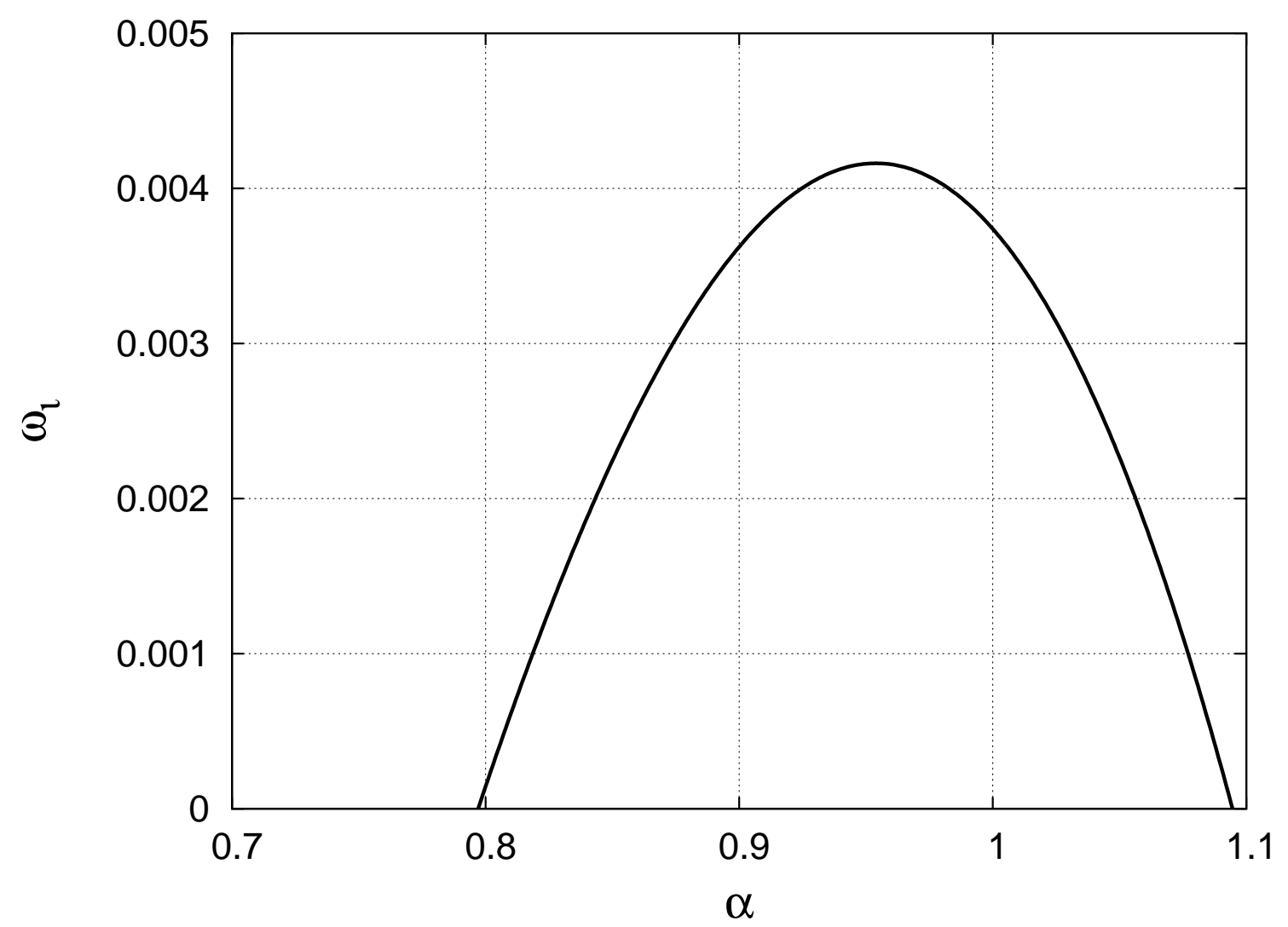

Figura 2.1: Taxa de amplificação temporal, referente ao modo simétrico, como função de $\alpha$ para um escoamento de Poiseulli para $R e=10^{4}$.

estacionária, uma vez que cresce e é chamada de instabilidade absoluta ${ }^{5}$.

\subsubsection{Teoria fracamente não linear}

A teoria linear de instabilidade hidrodinâmica é também a base para a teoria de instabilidade hidrodinâmica fracamente não linear (MEDEIROS, 2003). A teoria constitui uma correção à solução linear. O método considera que a solução geral é formada por uma expansão assintótica. O primeiro termo da série é a solução linear. A primeira correção, ou correção de primeira ordem, é a solução de uma equação linear e não homogênea, sendo que o termo não homogêneo é obtido a partir da solução linear. As correções de ordens superiores também vêm de equações lineares não homogêneas, nas quais o termo

\footnotetext{
${ }^{5}$ Uma característica distintiva da instabilidade absoluta é que ela se espalha para fora em ambas direções de uma só vez, pois quando a perturbação atinge um ponto, esta perturbação mantém-se crescente no tempo neste momento.
} 
não homogêneo é obtido a partir das soluções de ordem mais baixa. Claramente, a solução do problema linear é uma etapa do caso não linear.

\subsubsection{Teoria de estabilidade linear - Não-Local}

As equações de estabilidade parabolizadas ${ }^{6}$ (PSE) foram desenvolvidas por Bertolotti e Herbet (1991), Herbet (1997). Esta formulação resulta em um sistema de equações parabólicas que descrevem a evolução, na direção do escoamento, de instabilidades convectivas em escoamentos cisalhantes tais como camada limite, jatos e esteiras. A teoria leva em consideração efeitos não lineares e não paralelos em uma análise espacial, resultando em uma melhor representação da física do escoamento. Maiores detalhes podem ser consultados nos trabalhos de (BERTOLOTTI; HERBET, 1991; LI; MALIK, 1996; HERBET, 1997).

\subsubsection{Teoria de estabilidade linear - Global}

A diferença chave com a teoria linear clássica é que o espaço tri-dimensional é descrito por um domínio não homogêneo bi-dimensional e estendido periodicamente na $3^{a}$ direção espacial. A periodicidade dos modos é caracterizada pelo comprimento de onda $L_{z}$, associado com o número de onda $\beta$ tal que $L_{z}=2 \pi / \beta$. Perturbações no plano $O_{x y}$ são totalmente resolvidas, de forma que os modos são funções tri-dimensionais do espaço: não homogêneas nas direções $x$ e $y$ e periódica em $z$. Como tais, soluções do problema linear clássico de autovalor (teoria local) têm sido recuperadas como um caso limite de soluções fornecidas pela análise de estabilidade Global.

\section{BiGlobal}

Análise de estabilidade BiGlobal é baseada na descrição das variáveis como

$$
\mathbf{q}(x, y, z, t)=\underbrace{\overline{\mathbf{q}}(x, y)}_{\text {escoamento base }}+\varepsilon \underbrace{\tilde{\mathbf{q}}(x, y, z, t)}_{\text {perturbação }}, \varepsilon \ll 1,
$$

\footnotetext{
${ }^{6}$ ou do inglês Parabolized Stability Equations
} 
onde $\overline{\mathbf{q}}=(\bar{u}, \bar{v}, \bar{w}, \bar{T}, \bar{p})^{t}$ e $\tilde{\mathbf{q}}=(\tilde{u}, \tilde{v}, \tilde{w}, \tilde{T}, \tilde{p})^{t}$ para escoamento compressível, $\overline{\mathbf{q}}=(\bar{u}, \bar{v}, \bar{w}, \bar{p})^{t}$ e $\tilde{\mathbf{q}}=(\tilde{u}, \tilde{v}, \tilde{w}, \tilde{p})^{t}$ para escoamento incompressível.

Introduzindo (2.14), nas equações governantes para um escoamento incompressível (2.1) ou compressível $(2.3,2.4,2.5)$ subtraindo os termos de $\mathrm{O}(1)$ do escoamento base (que se cancelam identicamente) e extraindo os termos de ordem $\mathrm{O}\left(\varepsilon^{2}\right)$ obtemos as equações de Navier-Stokes linearizadas.

As perturbações $\tilde{\mathbf{q}}$ são introduzidas nas equações de Navier-Stokes linearizadas como

$$
\tilde{\mathbf{q}}(x, y, z, t)=\hat{\mathbf{q}}(x, y) \exp [i(\beta z-\omega t)]+\text { complexo conjugado, }
$$

e indicam que as perturbações, para uma análise temporal, possuem frequência complexa $\omega$, número de onda real $\beta=2 \pi / L_{z}$, onde $L_{z}$ é o comprimento de onda ao longo da direção transversal. Além disso, $\hat{\mathbf{q}}(x, y)=(\hat{u}, \hat{v}, \hat{w}, \hat{\theta}, \hat{p})^{t}$ (compressível) ou $\hat{\mathbf{q}}(x, y)=(\hat{u}, \hat{v}, \hat{w}, \hat{p})^{t}$ (incompressível) denotam as funções amplitudes.

Na estrutura temporal, $\beta$ é um parâmetro real e um problema de autovalor para o autovalor complexo $\omega$ é construído. A parte real de $\omega\left(\omega_{r}\right)$ está relacionada com a frequência do autovalor global, enquanto a parte imaginária $\omega_{i}$ é a taxa de crescimento/amortecimento. Um valor positivo de $\omega_{i}$ indica crescimento e o modo correspondente é temporalmente instável, enquanto $\omega_{i}<0$ indica amortecimento, e o modo é estável.

Esse processo de linerização das equações de Navier-Stokes e a introdução dos modos normais leva a um sistema de equações diferenciais parciais em termos das variáveis de perturbação e portanto um problema de autovalor.

O sistema, composto por 4 (incompressível) ou 5 equações (compressível), para a determinação do autovalor e da autofunção associada, na sua forma mais geral, pode ser escrito como um problema de autovalor complexo generalizado da forma

$$
\mathscr{L}(\overline{\mathbf{q}}) \hat{\mathbf{q}}=\omega \mathscr{R} \hat{\mathbf{q}}
$$


onde as matrizes $\mathscr{L}$ e $\mathscr{R}$ são obtidas apropriadamente e estão sujeitas às condições de contorno apropriadas. As soluções que seguem de (2.16) são chamados modos globais.

Escrevendo em detalhes a Eq. (2.16) para o caso incompressível temos:

$$
\begin{aligned}
{\left[\mathscr{L}_{i n c}-\bar{u}_{x}\right] \hat{u}-\bar{u}_{y} \hat{v}-\hat{p}_{x} } & =-i \omega \hat{u} \\
-\bar{v}_{x} \hat{u}+\left[\mathscr{L}_{\text {inc }}-\bar{v}_{y}\right] \hat{v}-\hat{p}_{y} & =-i \omega \hat{v} \\
-\bar{w}_{x} \hat{u}-\bar{w}_{y} \hat{v}+\mathscr{L}_{\text {inc }} \hat{w}-i \beta \hat{p} & =-i \omega \hat{w} \\
\hat{u}_{x}+\hat{v}_{y}+i \beta \hat{w} & =0
\end{aligned}
$$

onde

$$
\mathscr{L}_{\text {inc }}=\frac{1}{R e}\left(\frac{\partial^{2}}{\partial x^{2}}+\frac{\partial^{2}}{\partial y^{2}}-\beta^{2}\right)-\bar{u} \frac{\partial}{\partial x}-\bar{v} \frac{\partial}{\partial y}-i \beta \hat{w}
$$

Para o cálculo dos autovalores e respectivas autofunções é conveniente escrever as equações acima na forma matricial. Escrevendo o sistema de equações (2.17-2.20) em matriz por blocos, o operador linear para caso incompressível torna-se

$$
\left(\begin{array}{llll}
\mathscr{L}_{\mathrm{inc}}-\bar{u}_{x} & -\bar{u}_{y} & 0 & -\partial_{x} \\
-\bar{v}_{x} & \mathscr{L}_{\mathrm{inc}}-\bar{v}_{y} & 0 & -\partial_{y} \\
-\bar{w}_{x} & -\bar{w}_{y} & \mathscr{L}_{\mathrm{inc}} & -i \beta \\
\partial_{x} & \partial_{y} & i \beta & 0
\end{array}\right)\left(\begin{array}{l}
\hat{u} \\
\hat{v} \\
\hat{w} \\
\hat{p}
\end{array}\right)=\omega\left(\begin{array}{llll}
-\mathrm{i} & 0 & 0 & 0 \\
0 & -\mathrm{i} & 0 & 0 \\
0 & 0 & -\mathrm{i} & 0 \\
0 & 0 & 0 & 0
\end{array}\right)\left(\begin{array}{l}
\hat{u} \\
\hat{v} \\
\hat{w} \\
\hat{p}
\end{array}\right)
$$

Para o caso compressível lineariza-se a equação de estado e introduz-se no sistema de equações que descreve o escoamento compressível. O resultado disso é um problema de autovalor escrito nas variáveis nas variáveis $u, v, w$, temperatura $(\boldsymbol{\theta})$ e pressão da seguinte forma

$$
\mathscr{L}=\left(\begin{array}{ccccc}
\mathscr{L}_{x, \hat{u}} & \mathscr{L}_{x, \hat{v}} & \mathscr{L}_{x, \hat{w}} & \mathscr{L}_{x, \hat{\theta}} & \mathscr{L}_{x, \hat{p}} \\
\mathscr{L}_{y, \hat{u}} & \mathscr{L}_{y, \hat{v}} & \mathscr{L}_{y, \hat{w}} & \mathscr{L}_{y, \hat{\theta}} & \mathscr{L}_{y, \hat{p}} \\
\mathscr{L}_{z, \hat{u}} & \mathscr{L}_{z, \hat{v}} & \mathscr{L}_{z, \hat{w}} & \mathscr{L}_{z, \hat{\theta}} & \mathscr{L}_{z, \hat{p}} \\
\mathscr{L}_{e, \hat{u}} & \mathscr{L}_{e, \hat{v}} & \mathscr{L}_{e, \hat{w}} & \mathscr{L}_{e, \hat{\theta}} & \mathscr{L}_{e, \hat{p}} \\
\mathscr{L}_{c, \hat{u}} & \mathscr{L}_{c, \hat{v}} & \mathscr{L}_{c, \hat{w}} & \mathscr{L}_{c, \hat{\theta}} & \mathscr{L}_{c, \hat{p}}
\end{array}\right)
$$




$$
\mathscr{R}=\left(\begin{array}{ccccc}
\mathscr{R}_{x, \hat{u}} & 0 & 0 & 0 & 0 \\
0 & \mathscr{R}_{y, \hat{v}} & 0 & 0 & 0 \\
0 & 0 & \mathscr{R}_{z, \hat{w}} & 0 & 0 \\
0 & 0 & 0 & 0 & \mathscr{R}_{e, \hat{p}} \\
0 & 0 & 0 & \mathscr{R}_{c, \hat{\theta}} & \mathscr{R}_{c, \hat{p}}
\end{array}\right)
$$

Na descrição dos operadores (2.23) e (2.24) acima, o primeiro índice subscrito refere-se à equação de quantidade de movimento em sua direção correspondente, ie, $x, y, z, e$ refere-se à equação da energia e $c$ equação da continuidade. Já o segundo índice refere-se à variável de perturbação. Uma descrição completa dos coeficientes (matriz por bloco) de (2.23) e (2.24) encontra-se no apêndice (7.1).

Note que a formulação BiGlobal do problema de instabilidade permite a existência de uma distribuição arbitrária do escoamento ao longo das direções longitudinal $\bar{u}(x, y)$ e normal $\bar{v}(x, y)$. Isto é um contraste com a teoria linear clássica, que considera componentes de velocidade dependentes de apenas uma direção $\bar{u}(y)$ e $\bar{v}(y)$, i.e., escoamento paralelo. Como tal, a formulação BiGlobal do problema de instabilidade permite considerar todo o plano, e assim relaxar a hipótese de escoamento fracamente não-paralelo (CHOMAZ; HUERRE; REDEKOPP, 1988; CHOMAZ, 2005). 


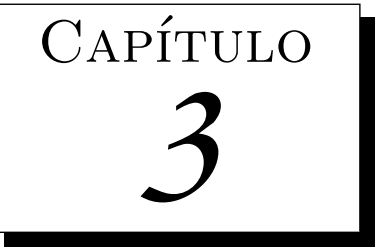

\section{Metodologia Numérica}

\subsection{Método de colocação espectral}

Métodos espectrais são ferramentas eficientes e precisas para resolver numericamente equações (CANUTO et al., 1988). Esta seção apresenta apenas um método espectral que foi utilizado no âmbito deste estudo como método de diferenciação numérica, além do método das diferenças finitas.

O método por colocação ${ }^{1}$ espectral de Chebyshev pode ser descrito como uma aproximação com base em polinômios interpolantes (neste trabalho polinômios de Chebyshev) que satisfazem a seguinte relação

$$
P_{N}\left(x_{j}\right)=f\left(x_{j}\right), j=1, \ldots, N
$$

onde $\left(x_{j}\right)_{j=1, \ldots, N}$ é uma discretização do intervalo em que a função está definida. Os coeficientes dos polinômios são definidos unicamente pelas $N$ relações (3.1).

O polinômio mais comum utilizado para interpolações talvez seja o de Lagrange

$$
P_{N}(x)=\sum_{i=0}^{N} f\left(x_{j}\right) c_{i}(x)
$$

\footnotetext{
${ }^{1} \mathrm{O}$ termo colocação aparece aqui como sinônimo de interpolação.
} 
onde

$$
c_{i}(x)=\prod_{j=0 \ldots N, j \neq i} \frac{x-x_{j}}{x_{i}-x_{j}}
$$

O polinômio $P_{N}$ interpola a função $f$ e por isso pode-se notar que

$$
c_{i}(x)= \begin{cases}1 & , \text { se } x=x_{i} \\ 0 & , \text { se } x=x_{j}\end{cases}
$$

O erro da interpolação Lagrangiana é proporcional a uma função cardinal

$$
\omega(x)=\prod_{j=0}^{N}\left(x-x_{j}\right)
$$

e pode ser demonstrado que este termo atinge um valor mínimo se $\left(x_{j}\right)$ correspondem aos zeros do polinômio de Chebyshev de grau $N+1$ (CANUTO et al., 1988).

O polinômio de grau $k$ é definido como

$$
T_{k}(x)=\cos (k \theta), \quad \theta=\arccos (x) .
$$

Estes zeros formam uma malha de Gauss no intervalo $[-1,+1]$

$$
x_{j}=\cos \left(\frac{j+\frac{1}{2}}{N+1} \pi\right), \quad j=0,1, \ldots, N .
$$

Os pontos são acumulados nas bordas do domínio. De fato, uma maneira de evitar erros numéricos associados ao cálculo das derivadas na região próxima à fronteira, é aumentar a densidade dos pontos nessa região, exatamente o que se obtém com a discretização de Gauss. Contudo, na discretização de Gauss os pontos da extremidade do intervalo não são incluídos. Com um erro de interpolação da mesma ordem de grandeza para uma malha de Gauss, pode ser preferível a introdução de uma malha de Gauss-Lobatto que é definida por

$$
x_{j}=\cos \left(\frac{\pi j}{N}\right), \quad j=0,1, \ldots, N
$$

Em grande parte deste trabalho foi utilizado uma malha computacional definida pelos pontos de colocação de Chebyshev-Gauss-Lobatto. De maneira gráfica, um exemplo desta 
malha é apresentado na figura (3.1). A figura evindencia a distribuição dos pontos e de que forma que os mesmos são acumulados nas bordas do domínio, como dito anteriormente.

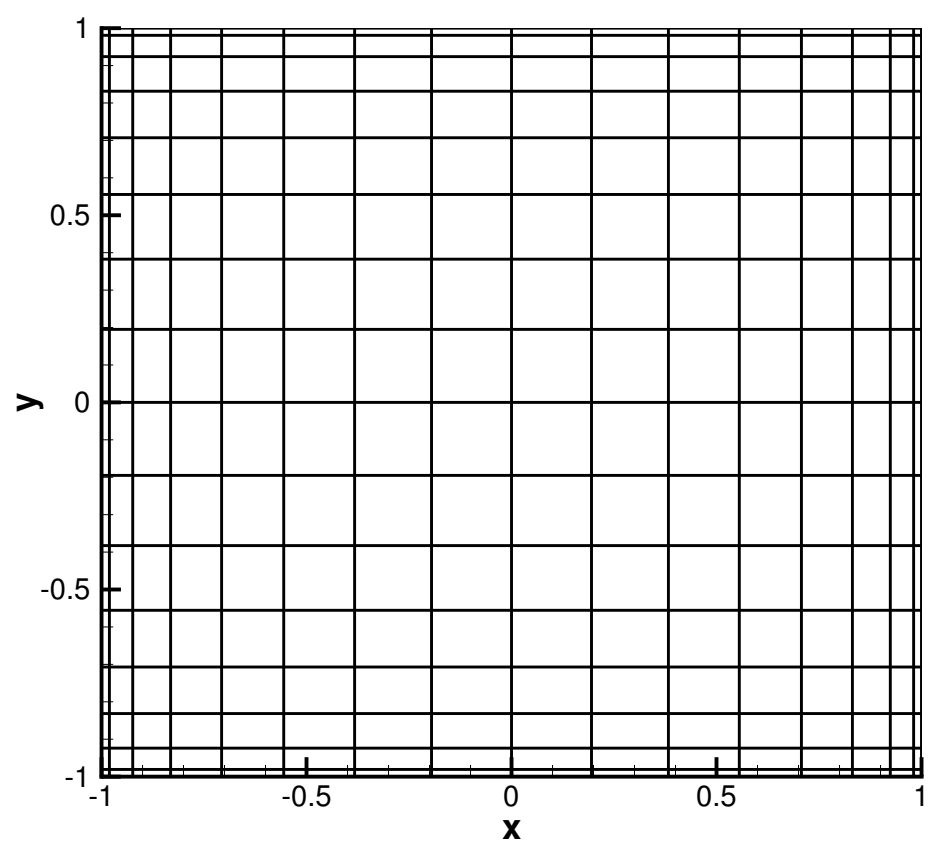

Figura 3.1: Malha computacional discretizada em pontos de Chebyshev-Gauss-Lobatto.

Definindo um mapeamento adequado, uma malha de Gauss-Lobatto pode ser usada para discretizar qualquer intervalo que se queira estudar. Uma descrição desses mapeamentos serão dados na seção (3.5).

O objetivo deste método é fornecer uma aproximação precisa das derivadas de uma função dada. Isto se deve ao fato de que as derivadas do polinômio interpolante podem ser obtidas de forma analítica. Esta função é interpolada pelo polinômio $P_{N}$. Pode ser notado que

$$
c_{i}(x)=\frac{\omega(x)}{\left(x-x_{j}\right) \omega^{\prime}\left(x_{j}\right)} .
$$

Depois da discretização de (3.3), alguns cálculos com a função cardinal definida por (3.5) produzem a seguinte aproximação de $f^{\prime}(x)$

$$
f^{\prime}(x) \approx \frac{d P_{N}}{d x}\left(x_{j}\right)=\sum_{k=0}^{N} \mathscr{D}_{j, k} g\left(x_{k}\right)
$$


onde a matriz da primeira derivada $\mathscr{D}$ é definida por

$$
\left(\mathscr{D}_{N}\right)_{i j}=\left\{\begin{array}{ll}
\frac{2 N^{2}+1}{6} & \text { se } i=j=0 \\
\frac{-x_{j}}{2\left(1-x_{j}^{2}\right)} & \text { se } i=j \neq 0, N \\
\frac{c_{i}}{c_{j}}(-1)^{i+j} & \text { se } i \neq j \\
-\frac{2 N^{2}+1}{6} & \text { se } i=j=N
\end{array} \quad \text { onde } c_{j}= \begin{cases}2 & \text { se } j=0, N \\
1 \quad \text { caso contrário }\end{cases}\right.
$$

Uma demonstração completa desta expressão pode ser encontrada em (CANUTO et al., 1988).

\subsection{Diferenças finitas com erro uniforme}

O método de discretização baseado em diferenças finitas está baseado na expansão em séries de Taylor para o cálculo aproximado das derivadas.

Utilizou-se um método apresentado por Hermanns e Hernandez (2008) para diferenças finitas com erro uniforme. Em linhas gerais, o método consiste em calcular uma aproximação numérica para um problema de valor de contorno em pontos discretos em um domínio computacional $[-1,+1]$ empregando aproximações por diferenças finitas para a primeira e segunda derivada espacial. Polinômios interpolantes lineares por partes são utilizados para construir os valores discretos da função no grid computacional. No entanto, pela sua seleção adequada é possível fazer o erro uniforme nessa interpolação polinomial por seccional e o resultado é um grid não-equispaceado, que é único para cada valor de $q$ (ordem) e $N$ (quantidade de pontos). Uma representação gráfica destes domínios pode ser visto nas figuras (3.2 a-b). Maiores detalhes podem ser vistos em (HERMANNS; HERNANDEZ, 2008). 


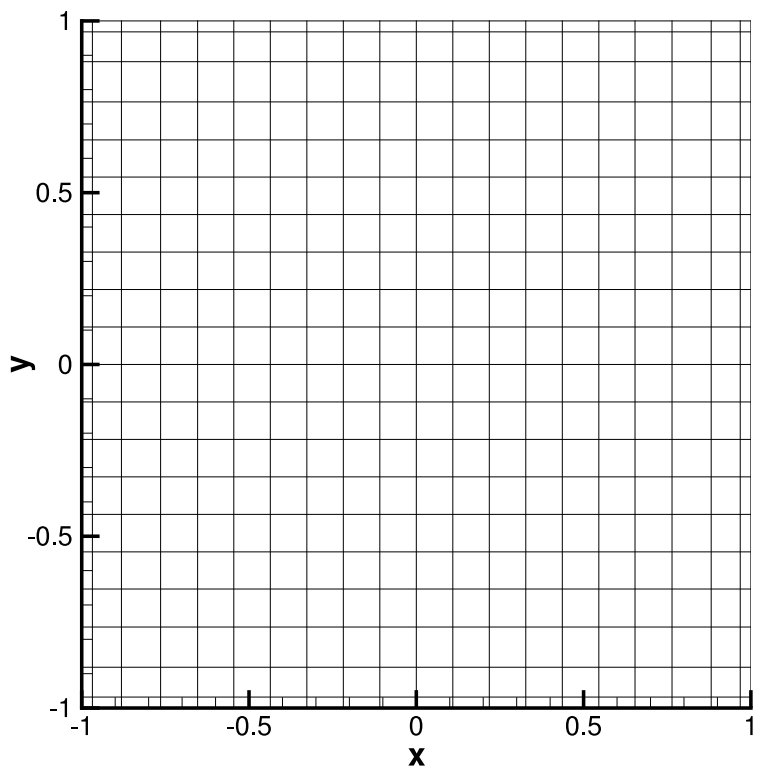

(a) ordem 6

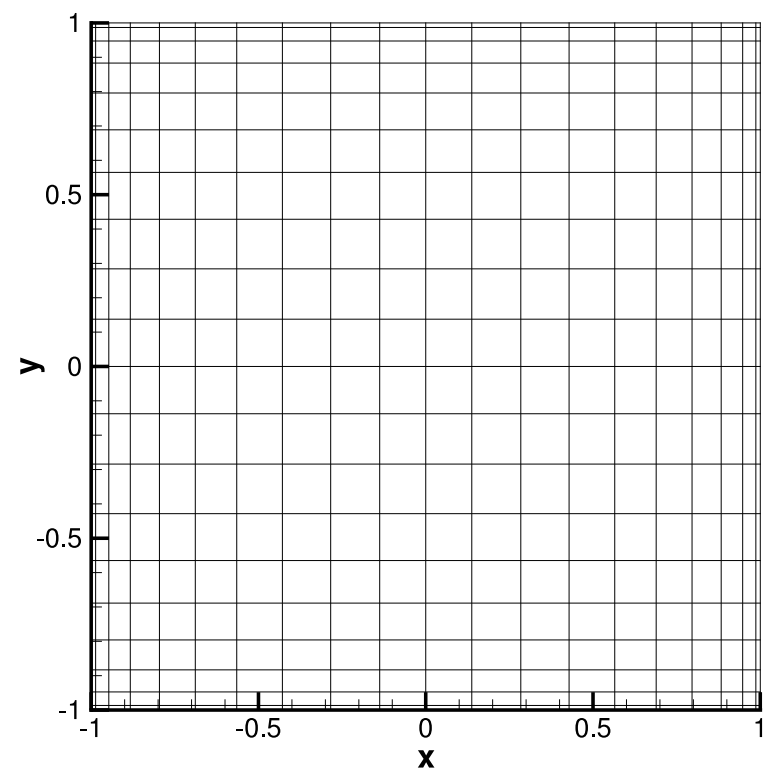

(b) ordem 18

Figura 3.2: Domínio computacional discretizado em $N_{x}=N_{y}=21$ pontos usando métodos das diferenças finitas com erro uniforme.

\subsection{Produto de Kronecker}

Se $A \in \mathrm{R}^{n_{A} \times m_{A}}$ e $B \in \mathrm{R}^{n_{B} \times m_{B}}$ então o produto de Kronecker $C=A \otimes B$, que pertence a $\mathrm{R}^{n_{A} n_{B} \times m_{A} m_{B}}$, é definido pela seguinte estrutura de blocos:

$$
C=\left(c_{i j}\right) \text { onde } c_{i j}=a_{i j} B \in \mathrm{R}^{n_{B} \times m_{B}}
$$

Esta operação entre matrizes é um produto tensorial que é uma operação algébrica bem conhecida (DAVIO, 1981).

Para exemplificar esta definição, sejam $A$ e $B$ matrizes tal que

$$
A=\left(\begin{array}{lll}
a_{11} & a_{12} & a_{13} \\
a_{21} & a_{22} & a_{23} \\
a_{31} & a_{32} & a_{33}
\end{array}\right) \quad B=\left(\begin{array}{ll}
\mathbf{b}_{11} & \mathbf{b}_{12} \\
\mathbf{b}_{21} & \mathbf{b}_{22}
\end{array}\right)
$$


O produto de Kronecker $C=A \otimes B$ é dado por

$$
C=\left(\begin{array}{cccccccc}
\mathrm{a}_{11} \mathbf{b}_{11} & \mathrm{a}_{11} \mathbf{b}_{12} & \mid & \mathrm{a}_{12} \mathbf{b}_{11} & \mathrm{a}_{12} \mathbf{b}_{12} & \mid & \mathrm{a}_{13} \mathbf{b}_{11} & \mathrm{a}_{13} \mathbf{b}_{12} \\
\mathrm{a}_{11} \mathbf{b}_{21} & \mathrm{a}_{11} \mathbf{b}_{22} & \mid & \mathrm{a}_{12} \mathbf{b}_{21} & \mathrm{a}_{12} \mathbf{b}_{22} & \mid & \mathrm{a}_{13} \mathbf{b}_{21} & \mathrm{a}_{13} \mathbf{b}_{22} \\
--- & --- & --- & --- & --- & --- & --- & --- \\
\mathrm{a}_{21} \mathbf{b}_{11} & \mathrm{a}_{21} \mathbf{b}_{12} & \mid & \mathrm{a}_{22} \mathbf{b}_{11} & \mathrm{a}_{22} \mathbf{b}_{12} & \mid & \mathrm{a}_{23} \mathbf{b}_{11} & \mathrm{a}_{23} \mathbf{b}_{12} \\
\mathrm{a}_{21} \mathbf{b}_{21} & \mathrm{a}_{21} \mathbf{b}_{22} & \mid & \mathrm{a}_{22} \mathbf{b}_{21} & \mathrm{a}_{22} \mathbf{b}_{22} & \mid & \mathrm{a}_{23} \mathbf{b}_{21} & \mathrm{a}_{23} \mathbf{b}_{22} \\
--- & --- & --- & --- & --- & --- & --- & --- \\
\mathrm{a}_{31} \mathbf{b}_{11} & \mathrm{a}_{31} \mathbf{b}_{12} & \mid & \mathrm{a}_{32} \mathbf{b}_{11} & \mathrm{a}_{32} \mathbf{b}_{12} & \mid & \mathrm{a}_{33} \mathbf{b}_{11} & \mathrm{a}_{33} \mathbf{b}_{12} \\
\mathrm{a}_{31} \mathbf{b}_{21} & \mathrm{a}_{31} \mathbf{b}_{22} & \mid & \mathrm{a}_{32} \mathbf{b}_{21} & \mathrm{a}_{32} \mathbf{b}_{22} & \mid & \mathrm{a}_{33} \mathbf{b}_{21} & \mathrm{a}_{33} \mathbf{b}_{22}
\end{array}\right)
$$

\subsection{Operador diferencial}

Os operadores diferenciais sobre uma malha computacional 2D são construídas baseado na matriz correspondente ao operador diferencial 1D e no produto de Kronecker, definido na seção (3.3).

A primeira derivada com respeito a $x$ e $y$ nos pontos da malha pode ser computada por

$$
\begin{aligned}
& \mathscr{D}_{x}=\mathscr{D} \otimes \mathscr{I} \\
& \mathscr{D}_{y}=\mathscr{I} \otimes \mathscr{D},
\end{aligned}
$$

onde $\mathscr{D}$ é a matriz derivada definidas por cada método de diferenciação apresentada e tem dimensões $N_{x} \times N_{x}$ e $N_{y} \times N_{y}$, respectivamente. Nas definições acima $\mathscr{I}$ é a matriz identidade.

As figuras 3.3 apresentam² a estrutura da matriz diferenciação para os métodos espectral (CGL) e diferenças finitas de ordem 8, para a primeira derivada com respeito a $x$ e y sobre um domínio computacional bi-dimensional.

\footnotetext{
${ }^{2}$ As figuras foram obtidas usando comando $\operatorname{spy}\left(\mathscr{D}_{x}\right)$ e $\operatorname{spy}\left(\mathscr{D}_{y}\right)$ do software Octave.
} 
Usando a mesma técnica, a segunda derivada é computada fazendo-se

$$
\begin{aligned}
& \mathscr{D}_{x x}=\mathscr{D}^{2} \otimes \mathscr{I} \\
& \mathscr{D}_{y y}=\mathscr{I} \otimes \mathscr{D}^{2} .
\end{aligned}
$$

Estruturalmente essas matrizes, $\mathscr{D}_{x x}$ e $\mathscr{D}_{y y}$, são iguais às correspondentes matrizes para a primeira derivada.

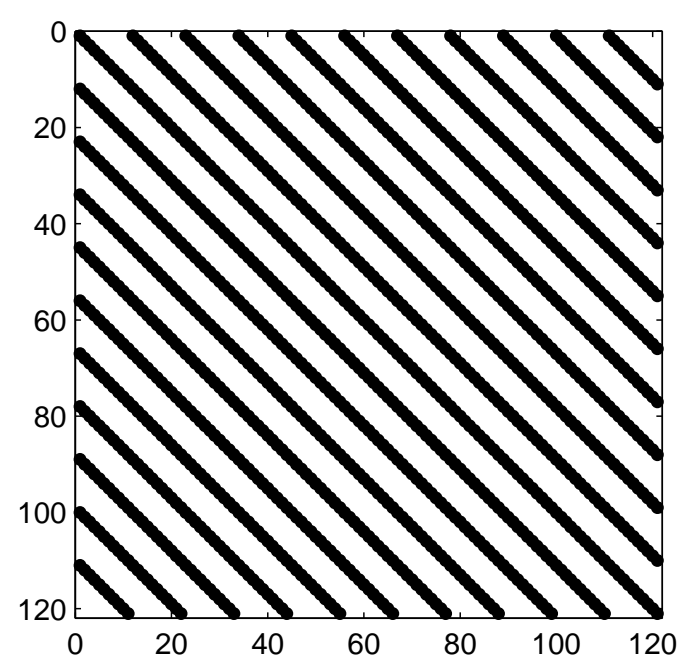

(a) $\mathscr{D}_{x}(C G L)$

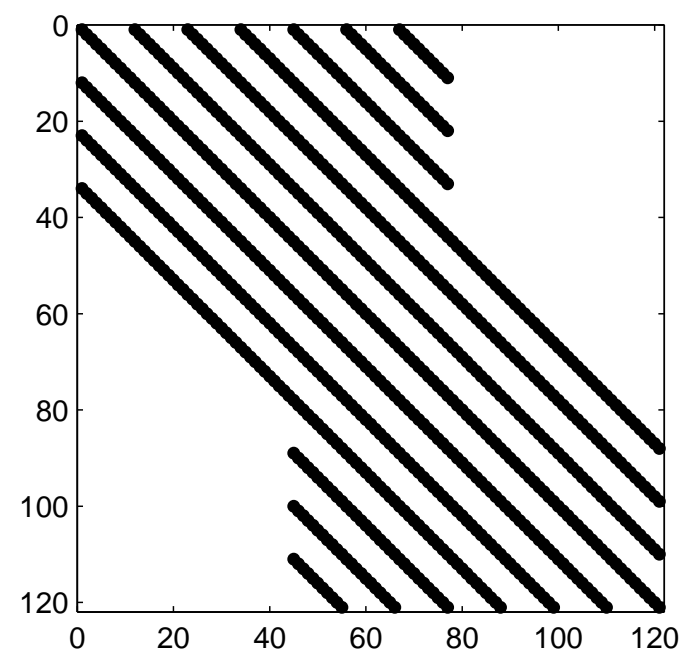

(c) $\mathscr{D}_{x}(D F-8)$

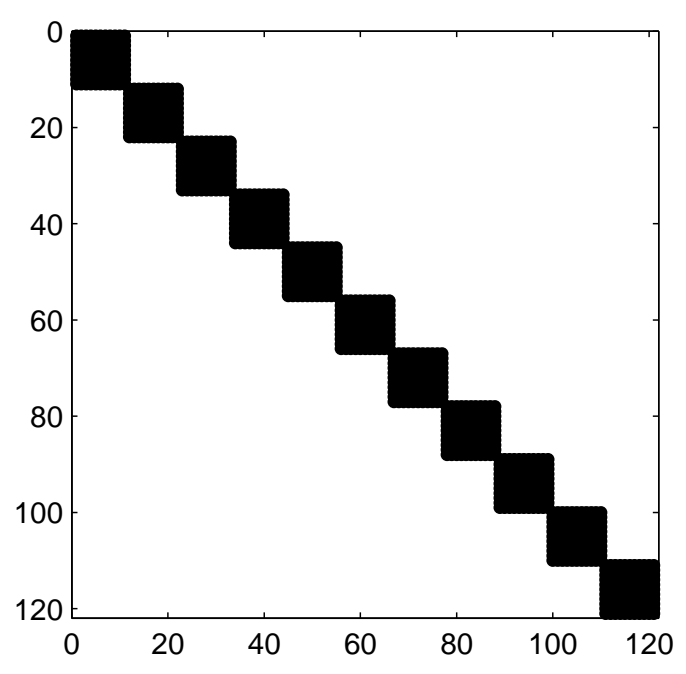

(b) $\mathscr{D}_{y}(C G L)$

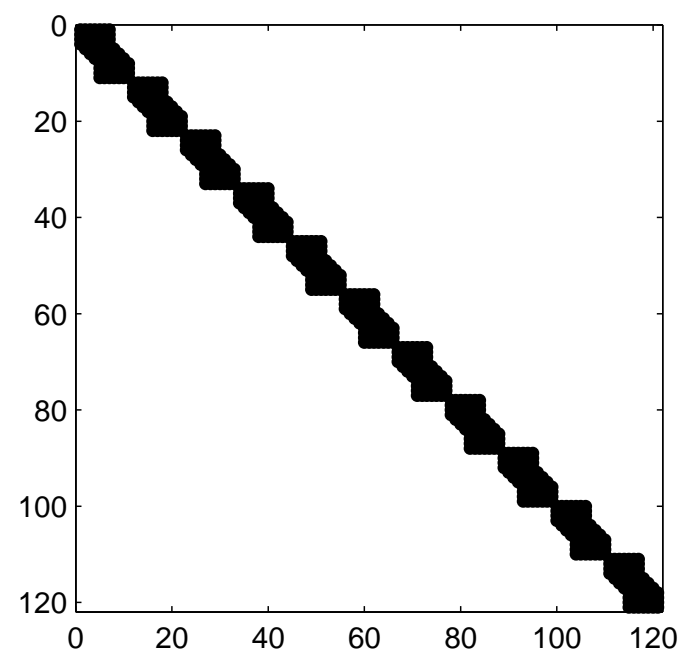

(d) $\mathscr{D}_{y}(D F-8)$

Figura 3.3: Estrutura da matriz diferenciação para um domínio computacional $N_{x}=N_{y}=$ 11. 
A derivada cruzada é computada da seguinte maneira:

$$
\mathscr{D}_{x y}=\mathscr{D}_{x} \times \mathscr{D}_{y}
$$

onde as definições das matrizes $\mathscr{D}_{x}$ e $\mathscr{D}_{y}$ foram apresentados anteriormente. A estrutura da matriz correspondente a derivada cruzada, para diferenças finitas de ordem 6 , pode ser visto na figura 3.4. Nota-se claramente uma grande diferença entre a estrutura apresentada por ambos os métodos. Por um lado, a matriz referente ao método por pontos de colocação é densa, e por outro, a estrutura apresentada na figura 3.4 .

A quatidade de elementos não-nulos dessa matriz tem uma influência direta na performance da resolução do problema de autovalor, como será apresentado em seções posteriores.

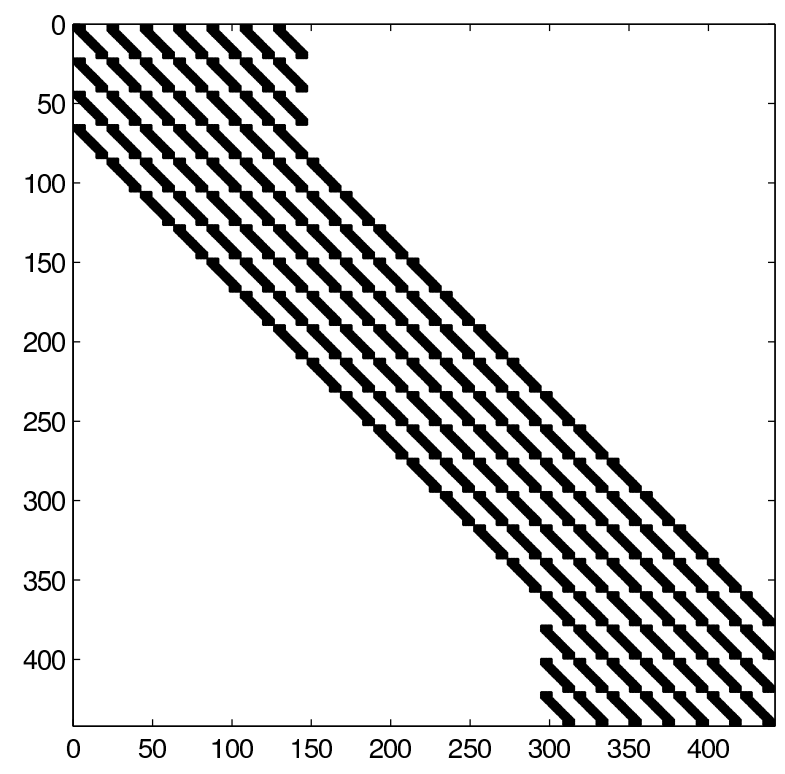

(a) $\mathscr{D}_{x y}(D F-6)$

Figura 3.4: Estrutura da matriz diferenciação para um domínio computacional $N_{x}=N_{y}=$ 21.

A tabela 3.1 apresenta fórmulas para cáculos dos elementos não-nulos correspondentes aos métodos diferenciação.

De forma mais geral, uma matriz derivada de ordem $m$ é então calculada por $\mathscr{D}^{(m)}=$ $\overbrace{\mathscr{D} \times \ldots \times \mathscr{D}}^{m-\text { vezes }}$, com $\times$ denotando produto de matriz. 
Tabela 3.1: Estimativa elementos não-nulos

\begin{tabular}{c|c|c}
\hline & CGL & DF-q \\
\hline $\mathscr{D}_{x}$ & $N_{x}^{2} \times N_{y}$ & $\left(N_{x} \times N_{y}\right) \times(q+1)$ \\
$\mathscr{D}_{y}$ & $N_{x} \times N_{y}^{2}$ & $\left(N_{x} \times N_{y}\right) \times(q+1)$
\end{tabular}

A quantidade de elementos não-nulos para as matrizes $\mathscr{D}_{x x}$ e $\mathscr{D}_{y y}$ é a mesma para as respectivas matrizes $\mathscr{D}_{x}$ e $\mathscr{D}_{y}$.

\subsection{Mapeamento da Malha Computacional}

As matrizes derivadas tem sido introduzidas usando um grid computacional definido no intervalo $[-1,+1]$ e, para problemas que podem ser definidos neste intervalo, a escolha pela discretização espacial em pontos de Gauss-Lobatto é adequada. Entretanto, existem problemas físicos que estão definidos em outros intervalos, por exemplo $[0, \infty)$. Dessa forma, uma função de mapeamento se faz necessária para levar os pontos do intervalo $[-1,+1]$ para $[0, \infty)$. Muitas transformações tem sido consideradas e umas delas, muito usada para domínio semi-inifito, é definida por $^{3}$

$$
\eta_{i}=l \frac{1-x_{i}}{1+s+x_{i}}
$$

onde $l=\frac{\eta_{\infty} \eta_{\frac{1}{2}}}{\eta_{\infty}-2 \eta_{\frac{1}{2}}}, s=\frac{2 l}{\eta_{\infty}}$ e $\left(x_{i}\right)$ são os pontos de colocação de Gauss-Lobatto definido por (3.8). Outros mapeamentos podem ser encontrados em (BOYD, 1989).

Para esta transformação, dois parâmetros devem ser escolhidos. $\eta_{\infty}$ é o limite superior do domínio computacional e $\eta_{\frac{1}{2}}$ é um valor abaixo do qual estão acumulados metade dos pontos da malha. Esta é em geral a região de interesse.

Assim, a matriz derivada $\mathscr{D}(x)$ torna-se $\mathscr{D}(\eta)$ que é definida por

$$
\begin{aligned}
\mathscr{D}_{i, j}(\eta) & =\frac{d x_{i}}{d \eta_{i}} \mathscr{D}_{i, j}(x), \\
\mathscr{D}_{i, j}^{2}(\eta) & =\left(\frac{d x_{i}}{d \eta_{i}}\right)^{2} \mathscr{D}_{i, j}^{2}(x)+\frac{d^{2} x_{i}}{d \eta_{i}^{2}} \mathscr{D}_{i, j}(x),
\end{aligned}
$$

\footnotetext{
${ }^{3}$ Utilizou-se uma direção do domínio para se definir a transformação (3.20), entretanto a mesma poderia ser definida para uma direção arbitrária, sem perda de generalidade.
} 


\subsection{Método de Arnoldi}

As técnicas mais efecientes para resolver um problema de autovalor generalizado são baseadas em métodos iterativos de projeções. Esse métodos utilizam muitas vezes o subespaço de Krylov para buscar soluções de sistemas lineares do tipo $A x=b$ (SAAD, 2000).

Especificamente, para os problemas de instabilidade BiGlobal, as abordagens mais utilizadas tem sido o algorítmo de Arnoldi (NAYAR; ORTEGA, 1993; THEOFILIS, 2003; THEOFILIS, 2011) e que faz parte dos diversos métodos de iteração do subespaço de Krylov apresentados por (SAAD, 1980). O tempo de computação depende linearmente da dimensão do subespaço.

Nota-se um grande uso do algorítmo de Arnoldi em diversas aplicações da engenharia e a razão seja provavelmente pela simplificidade e eficiência de seu uso e de sua implementação. Dentro do contexto deste trabalho, encontramos diversos trabalhos que empregaram o métodos de Arnoldi, a saber: Natarajan (1992), Nayar e Ortega (1993), Natarajan e Acrivos (1993), Ehrenstein (1996), Theofilis (2000), Lehoucq e Salinger (2001), Crouch, Garbaruk e Magidov (2007a), Giannetti e Luchini (2007), Sipp e Lebedev (2007), Kitsios et al. (2009), Gennaro et al. (2011a), Gennaro et al. (2011b).

Como todos os algorítmos da classe Krylov, o elemento principal do processo iterativo de Arnoldi é a geração de um subespaço de Krylov, $K_{m}$, de dimensão $m$.

Um subespaço de Krylov é definido por:

$$
K_{m}=\operatorname{span}\left\{\mathbf{q}_{0}, \mathbf{M} \mathbf{q}_{0}, \mathbf{M}^{2} \mathbf{q}_{0}, \ldots, \mathbf{M}^{m-1} \mathbf{q}_{0}\right\}
$$

onde $\mathbf{M}$ é a matriz discretizada e $\mathbf{q}_{0}$ um vetor arbitrário qualquer.

A idéia fundamental é que o conjunto de vetores gerados pela iteração, que abrange o subespaço de Krylov, seja relativamente pequeno e de forma que os autovalores obtidos seja uma boa solução aproximada do autovalores do problema original (cálculo do espectro 
total). Uma descrição desse método pode ser visto no apêndice 7.2.

Os problemas em geral são de muitas ordens de grandeza. Por isso, um alto custo computacional está associado nas soluções dos problemas e as restrição computacionais, muitas vezes, inviabilizam a resolução do problema.

Do ponto de vista da análise de estabilidade linear, os autovalores mais importantes são aqueles próximos ao eixo $\omega_{r}=0$. Como visto em estudos anteriores (DING; KAWAHARA, 1998), somente autovalores com grande módulo podem ser obtidos pela aplicação direta do algorítmo e oferecem somente uma janela do autoespectro. Quando os autovalores próximos ao eixo imaginário são procurados, necessita-se de uma transformação simples usada com objetivo de converter o problema original em um problema onde os autovalores desejados tenham grande módulo. Para o cálculo da parte desejada do autoespectro, utilizou-se uma transformação conhecida como shift-and-invert. Note que os autovetores não são afetados por essa transformação.

O processo de Arnoldi é um método para computar uma base ortornormal $\left\{v_{1}, v_{2}, \ldots, v_{j}\right\}$ do subespaço de Krylov, $K_{j}\left(A, v_{1}\right)$. Ortogonalização foi obtida através de uma procedimento muito conhecido por Gram-Schmidt.

Algorítmo de Arnoldi:

1 Escolher um vetor unitário $v_{1}$ e normalizá-lo

2 For $j=1,2, \ldots, m$

3 Computar $w_{i j}:=A v_{j}$

$4 \quad$ Do $i=1, \ldots, j$

$5 \quad h_{i j}=\left(w_{j}, v_{i}\right)$

$6 \quad w_{j}=w_{j}-h_{i j} v_{i}$

$7 \quad$ EndDo 


$$
\begin{array}{cc}
8 & h_{j+1, j}=\left\|w_{j}\right\|_{2} \\
9 & v_{j+1}=w_{j} / h_{j+1, j}
\end{array}
$$

10 EndDo

Este algorítmo fornece uma base ortonormal $\mathbf{V}_{m}=\left[v_{1}, v_{2}, \ldots, v_{m}\right]$ do subespaço de Krylov $K_{m}=\operatorname{span}\left\{v_{1}, C v_{1}, C^{2} v_{1}, \ldots, C^{m-1} v_{1}\right\}$. A restrição de $C$ à $K_{m}$ é representada pela matriz de Hessenberg $\mathbf{H}_{m}=\left\{h_{i j}\right\}$. Os autovalores dessa matriz são uma aproximação dos $m$ autovalores de maior módulo do problema original. Os autovatores associados à estes autovalores podem ser obtidos de

$$
\hat{q}_{i}=\mathbf{V}_{m} \tilde{y}_{i}
$$

onde $\tilde{y}_{i}$ é um autovetor de $\mathbf{H}_{m}$ associado com o $\mu_{i}$-th autovalor.

Note que, uma vez que a matriz não é conhecida a priori, um sistema linear nãosimétrico

$$
C v_{j}=A^{-1} B v_{j}=q_{j}
$$

ou ainda,

$$
A q_{j}=B v_{j}
$$

deve ser resolvido a cada iteração, $q_{j}$ é um vetor auxiliar desconhecido. Uma observação importante é que para este processo é necessário uma inversão do operador, o que significa pelo menos uma decomposição LU da matriz deve ser feita.

O tempo total necessário para uma análise completa de Arnoldi depende muito da eficiência da resolução do sistema linear descrito acima, grau de esparsidade, bem como da dimensão do subespaço de Krylov $m$ utilizado para aproximar os autovalores mais importantes.

A figura (3.5) apresenta os autovalores calculados para um problema de cavidade $\left(R e=782, \beta=15.3\right.$ e $\left.M a=10^{-4}\right)$ ressaltando esse "enjanelamento"dito acima. Na sobreposição entre os autovalores obtidos pelos métodos QZ e algorítmo de Arnoldi nota-se claramente uma excelente concordância somente para os primeiros autovalores. O aumento 
da dimensão do subespaço de Krylov proporcionaria uma maior concordância entre os autovalores obtidos por ambos métodos. Em geral, como o interesse está nos autovalores mais instáveis (ou menos estáveis) o método é adequado para obtê-los.

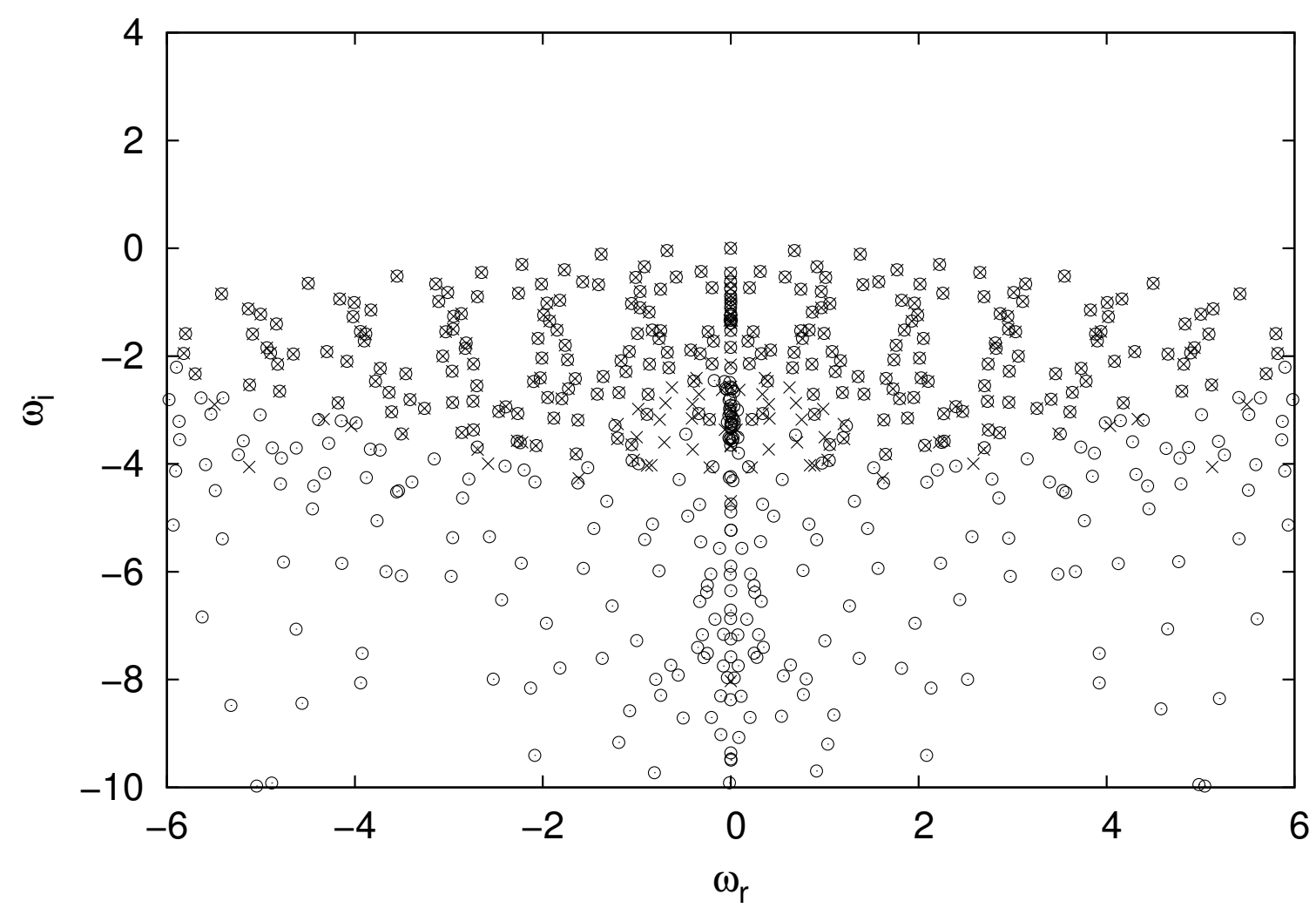

Figura 3.5: Autovalores obtidos para o escoamento em uma cavidade. Símbolos "X"representam os autovalores obtidos pelo algorítmo de Arnoldi e os símbolos "o" são os autovalores obtidos pelo método QZ.

\subsection{Solução do Problema de Autovalor por Matriz Esparsa}

O desenvolvimento do algorítmo esparso está baseado na construção e no armazenamento da matriz esparsa. Primeiramente, uma vez escolhido o tamanho do domínio computacional e a resolução, a discretização é realizada neste trabalho em pontos de Gauss-Lobatto (seção 3.1) e diferenças finitas em ambas as direções $x$ e $y$. Para resolver adequadamente as regiões de interesse, utilizou-se estiramento e os mapeamentos de malha 
adequados apresentados na seção 3.5. A matriz do problema de autovalor generalizado resultante da discretização dos operadores $\mathscr{L}$ e $\mathscr{R}$ foi resolvida usando uma implementação shift-and-invert do método de Arnoldi (seção 3.6).

O poblema de autovalor generalizado 2.16 pode ser transformado em um problema de autovalor canônico tomando um parâmetro $\sigma$ de descolamento tal que

$$
(\mathscr{L}-\sigma \mathscr{R}) \hat{\mathbf{q}}=(\omega-\sigma) \mathscr{R} \hat{\mathbf{q}}
$$

ou ainda

$$
\frac{1}{\omega-\sigma} \hat{\mathbf{q}}=(\mathscr{L}-\sigma \mathscr{R})^{-1} \mathscr{R} \hat{\mathbf{q}}
$$

Assim temos um "novo"problema de autovalor

$$
\overline{\mathscr{L}} \hat{\mathbf{q}}=\mu \hat{\mathbf{q}}
$$

onde $\overline{\mathscr{L}}=(\mathscr{L}-\sigma \mathscr{R})^{-1} \mathscr{R}, \mu=(\omega-\sigma)^{-1}$, e com isso o problema torna-se mais adequado para o uso do algorítmo de Arnoldi na busca da solução do problema.

A decomposição LU da matriz $(\mathscr{L}-\sigma \mathscr{R})$ é a mais exigente em termos de tempo e memória computacional.

A matriz resultante da discretização dos operadores $\mathscr{L}$ e $\mathscr{R}$ contém um grande número de elementos nulos, como pode ser visto nas figuras (3.6a-f) e (3.7a-c). Observe que a discretização do operador $\mathscr{R}$, lado direito do problema de autovalor, possuem a mesma estrutura esparsa, já que não há termos que dependam de derivadas. Nestas figuras, utilizou-se 2 métodos de discretização para os operadores apresentados para os problemas de Poisson (1 variável), incompressível (4 variáveis) e compressível (5 variáveis), a saber, métodos spectral e diferenças finitas. Os tamanhos das matrizes foram mantidos de acordo com o caso compressível no intuito de se notar a complexidade crescente dos problemas devido ao incremento de variáveis. É notável ainda o grande aumento na esparsidade que a discretização em diferenças finitas apresenta em relação à discretização em pontos de Chebyshev-Gauss-Lobatto (CGL). Outros aspectos numéricos relacionados as duas 
abordagens serão discutidos juntamente com os casos estudados.

Apesar de apresentarem padrões de esparsidade distintas, os dois métodos de discretização sugerem o uso de técnicas esparsas a fim de reduzir tanto a memória bem como o tempo computacional requerido na decomposição LU. Consequentemente, duas abordagens distintas do algorítmo foram implementadas aqui e testadas: uma diz respeito a construção do operador linear em matriz densa e usa rotinas LAPACK para a fatoração LU de uma matriz geral. A outra abordagem é esparsa e usa pacote de bibliotecas MUMPS ${ }^{4}$ (AMESTOY et al., 2001; AMESTOY et al., 2006) para a fatoração LU e a solução do sistema linear. Ambas as abordagens realizam a decomposição LU completa, ou seja, por métodos diretos. Na abordagem esparsa, as operações aritméticas foram realizadas com as matrizes em formato $\mathrm{CSR}^{5}$ (SAAD, 2000). No apêndice 7.4 pode ser visto uma matriz no formato CSR.

O sistema linear é resolvido pelo MUMPS em três etapas: fase de análise (fatoração simbólica), fatoração $L U$ e solução, descritos resumidamente a seguir. A matriz original é armazenada na forma de três vetores, sendo que o primeiro e o segundo armazenam os índices referentes a linha e coluna dos elementos não nulos, e o terceiro armazena os valores. Esta forma de armazenamento é conhecida como formato coordenadas. Uma vez construídos, eles são analisados durante a fase de análise, que retorna uma estimativa da memória necessária para realizar a decomposição $L U$. Esta estimativa depende das operações a serem executadas durante a decomposição em $L U$, sobretudo operações do tipo

$$
a(i, j):=a(i, j)-[a(i, k) a(k, j)] / a(k, k) .
$$

Consequentemente, se $a(i, k), a(k, j)$ e $a(k, k)$ são elementos não-nulos, $a(i, j)$ será atualizado como não-nulo também, mesmo que a seu valor na matriz original fosse igual a zero. Tais coeficientes são chamados de coeficientes de preenchimento. ${ }^{6}$ A quantidade destes

\footnotetext{
${ }^{4}$ MUltifrontal Massively Parallel Solver

${ }^{5}$ Siglas correspondem ao nome em inglês, Compressed Sparse Row. Adotou-se e termo dessa maneira pelo seu amplo uso e maior identificação dentre as bibliotecas que existem, como MKL e SPARSEKIT (http://people.sc.fsu.edu/ jburkardt/f_src/sparsekit/sparsekit.html)

${ }^{6}$ fill-in coefficient
} 
coeficientes depende de vários aspectos, entretanto, o mais importante deles é a ordem em que os pivôs são eliminados pelo método de ordenação. Ao final da fase de análise, MUMPS retorna a estimativa da memória necessária: se um grande número de coeficientes de preenchimento é retornado, benefícios limitados do uso de técnicas esparsas devem ser esperado. A ordenação dos elementos da matriz para reduzir a quantidade dos coeficientes de preenchimento dentro de MUMPS pode ser fornecido por vários métodos: Approximate Minimum Degree (AMD)(AMESTOY; DAVIS; DUFF, 1996), Approximate Minimum Fill (AMF), além de PORD (SCHULZE, 2001) e METIS $^{7}$ (KARYPIS; KUMAR, 1998). O uso destes métodos será exemplificado mais adiante. Em resumo, o procedimento usando matriz esparsa consiste em quatro etapas em oposição as duas no caso denso:

1. etapa de ordenação que reordena as linhas e colunas minimizando os coeficientes de preenchimento.

2. fatoração simbólica em que MUMPS determina as estruturas diferentes de zero dos fatores e cria estruturas adequadas para os fatores dados calculados no item anterior.

3. fatoração numérica que calcula a matriz L e os fatores de U.

4. solução sistema linear (dentro do algorítmo de Arnoldi) usando os fatores calculados.

\footnotetext{
${ }^{7}$ Um conjunto de programas em serial (ParMETIS - versão paralela) para particionamento de grafos, malhas de elementos finitos, e redução dos coeficientes de preenchimento de matrizes esparsas.
} 


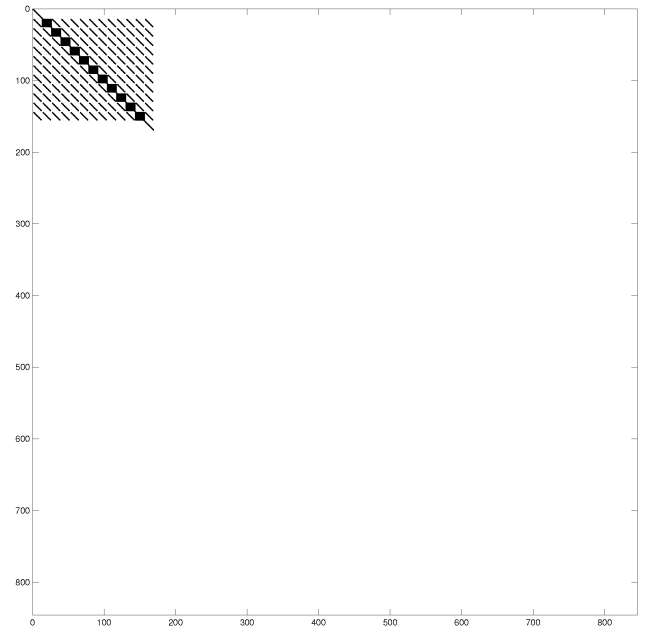

(a) Poisson - discretização espacial em pontos de Chebyshev-Gauss-Lobatto

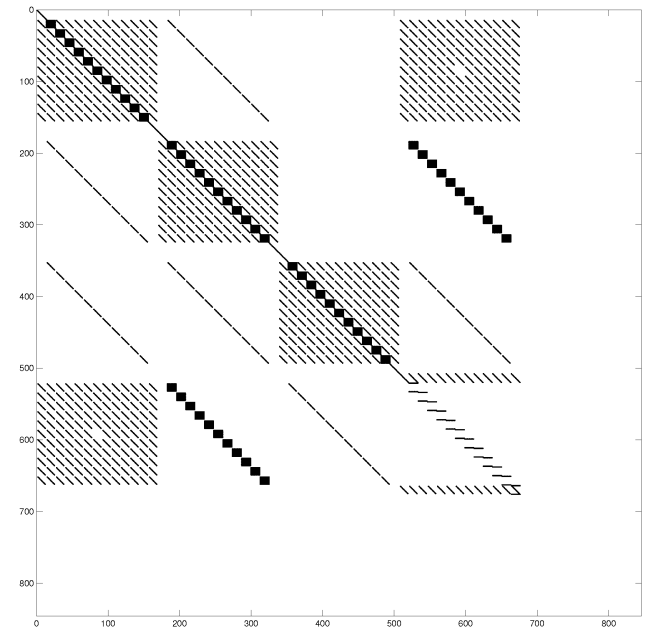

(c) Incompressível - discretização espacial (d) Incompressível - discretização espacial em pontos de Chebyshev-Gauss-Lobatto
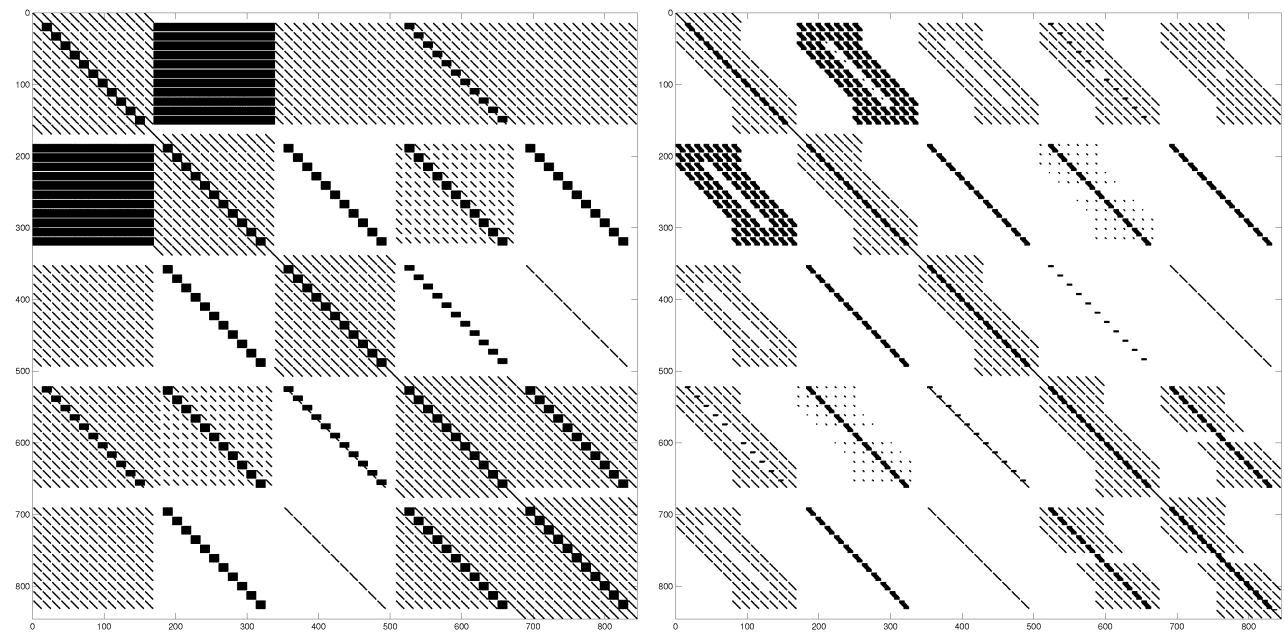

(e) Compressível - discretização espacial (f) Compressível - discretização espacial em pontos de Chebyshev-Gauss-Lobatto em diferenças finitas de ordem 6

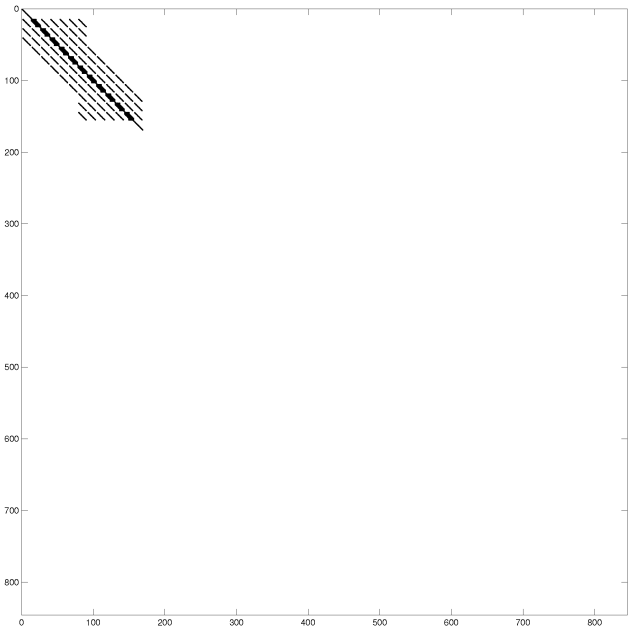

Poisson - discretização espacial em diferenças finitas de ordem 6 .

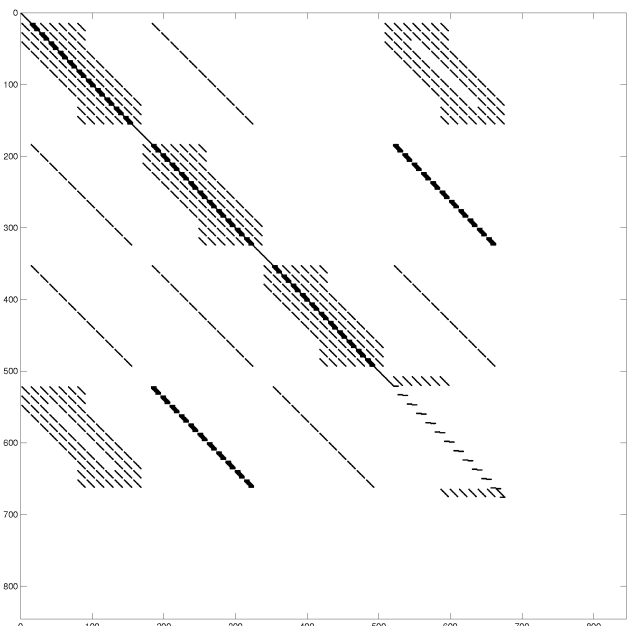




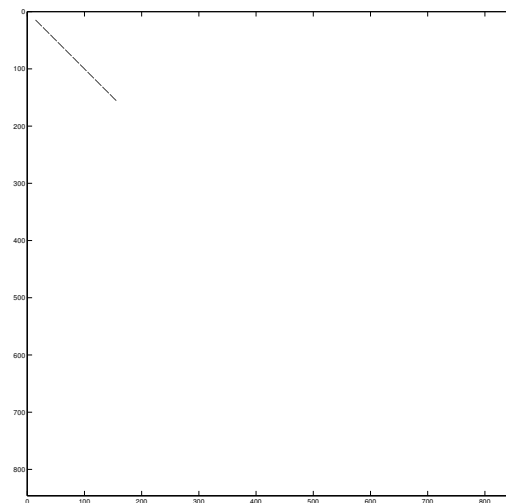

(a) Poisson

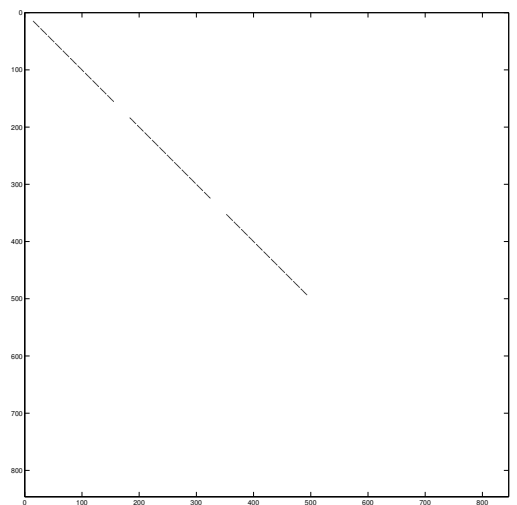

(b) Incompressível

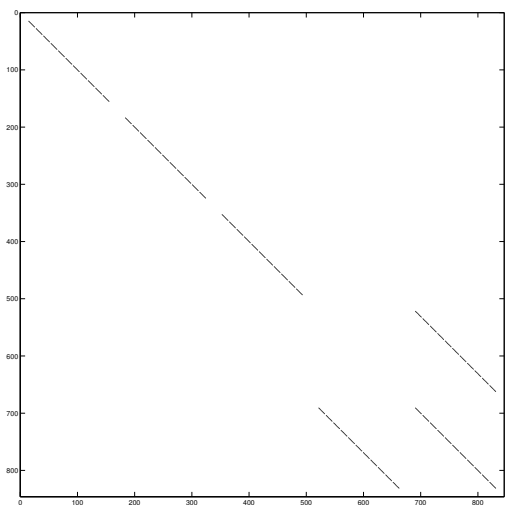

(c) Compressível

Figura 3.7: Padrão de esparsidade do operador $\mathscr{R}$ referente aos problemas de Poisson, escoamento incompressível e compressível, discretizados em $N x=N y=13$ para ambos métodos. 
CAPÍTULO

\section{Casos para verificação e desenvolvimento do}

\section{código}

\subsection{Problema de Autovalor de Laplace}

Considere um problema de autovalor de Laplace bi-dimensional

$$
-\nabla^{2} u+f(x, y) u=\lambda u
$$

definido no domínio $\Omega \equiv(x, y) \in[-1,1] \times[-1,1]$ e sujeito às condições de contorno de Dirichlet $u=0$.

Considerando $f \equiv 0$, existe uma solução analítica para o problema obtida através do método de separação de variáveis e tem a seguinte forma:

$$
\sin \left(k_{x}(x+1)\right) \sin \left(k_{y}(y+1)\right)
$$

onde $k_{x}$ e $k_{y}$ são múltiplos inteiros de $\pi / 2$, com os autovalores dados por:

$$
\frac{\pi^{2}}{4}\left(i^{2}+j^{2}\right), i, j=1,2,3,4, \ldots
$$

A tabela 4.1 apresenta uma comparação entre os resultados exatos e numéricos, além disso, os autovetores correspondentes são mostrados nas figura (4.1). Foram necessários 
12 pontos de colocação Chebyshev na discretização espacial para obter uma excelente concordância com a solução exata.

Tabela 4.1: Comparação numérica do problema bi-dimensional de autovalor de Laplace discretizado em 12 pontos de Chebyshev-Gauss-Lobatto.

\begin{tabular}{ccc}
\hline Modo & Solução Analítica & Presente trabalho \\
\hline 1 & 4.9348022005422 & 4.93480220054246 \\
2 & 12.3370056042652 & 12.3370056042653 \\
3 & 12.3370056042652 & 12.3370056042653 \\
4 & 19.7392090079877 & 19.7392090079874 \\
\hline
\end{tabular}

Tabela 4.2: Comparação numérica dos autovalores obtidos pelo presente trabalho. O problema foi discretizado em 24 pontos CGL.

\begin{tabular}{ccc}
\hline Modo & (TREFETHEN, 2000) & Presente trabalho \\
\hline 1 & 5.22149136352 & 5.22149136351 \\
2 & 12.3949457924 & 12.39494579240 \\
3 & 13.6890743205 & 13.68907432056 \\
4 & 21.3233577871 & 21.32335778717 \\
\hline
\end{tabular}
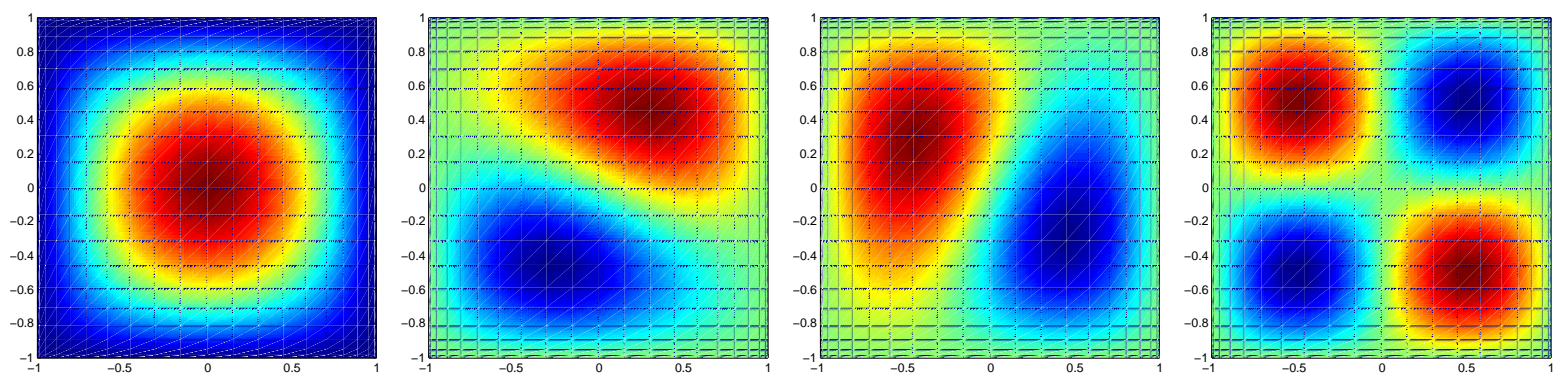

Figura 4.1: Autovetores correspondentes aos 4 primeiros modos associados ao problema de Laplace bi-dimensional homogêneo.

A figura 4.2 apresenta o comportamento do erro numérico para distintas discretizações espaciais da equação 4.1 para o modo 34. Observa-se uma rápida convergência das soluções numéricas calculadas e a quantidade pequena de pontos na discretização, mesmo para uma boa concordância no cálculo dos modos que não são os primeiros.

Agora, considere o problema de autovalor (4.1) com $f \neq 0$ definida por:

$$
-\nabla^{2} u+e^{(20(y-x-1))} u=\lambda u
$$




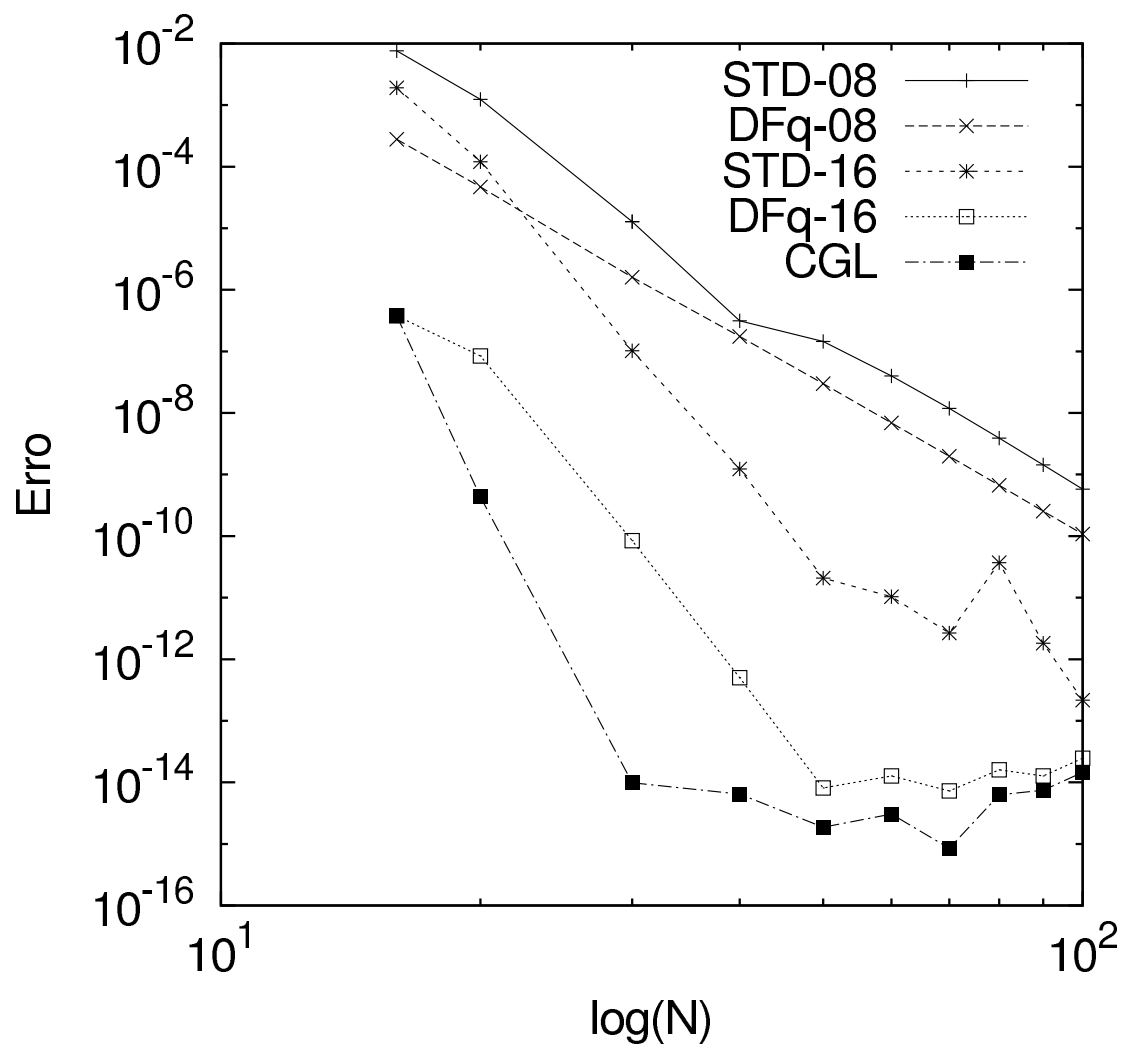

Figura 4.2: Erro numérico associado ao cálculo do modo 34 que corresponde à $i=5 \mathrm{e}$ $j=3$ na solução (4.3) em distintos métodos e ordem de discretização espacial: diferenças finitas padrão (STD) e com erro uniforme (DFq) e pontos de Chebyshev-Gauss-Lobatto.

definido no mesmo domínio sujeito às mesmas condições de contorno do caso anterior.
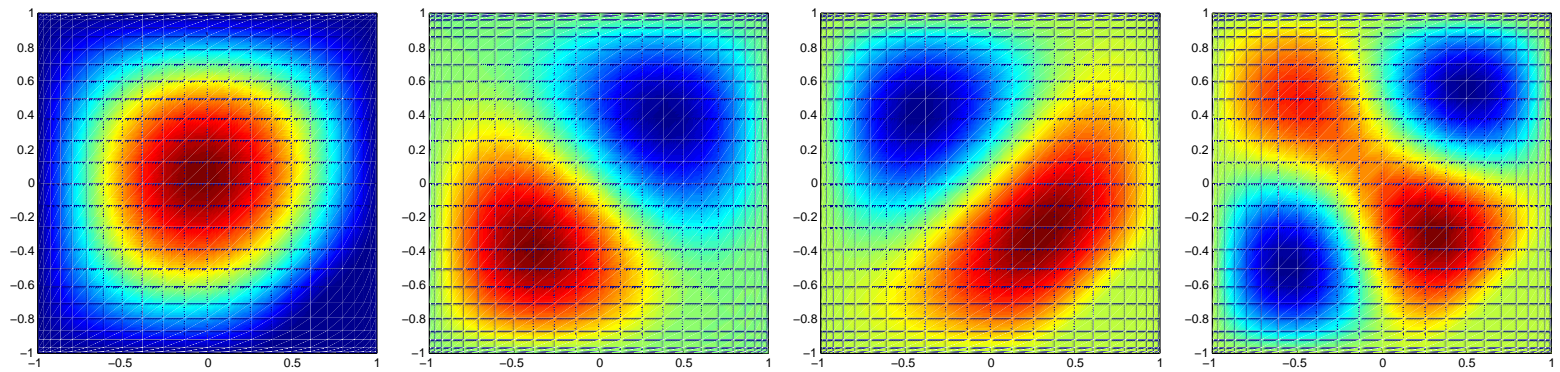

Figura 4.3: Autovetores correspondentes aos 4 primeiros modos associados ao problema de Laplace bi-dimensional em que $f(x, y)=e^{(20(y-x-1))}$.

A tabela 4.1 apresenta resultados obtidos pelo código desenvolvido em comparação com os resultados obtidos por Trefethen (2000). Nota-se claramente uma excelente concordância entre os resultados. Os autovetores correspondentes aos 4 primeiros modos podem ser visto na figura 4.3. 


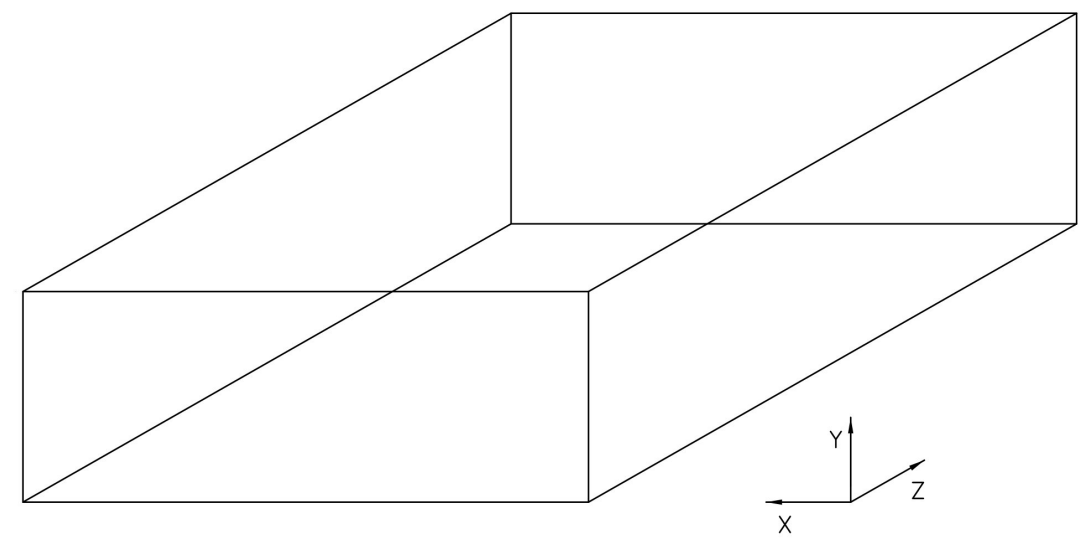

Figura 4.4: Esquema geométrico de um duto retangular.

\subsection{Duto Retangular}

Tatsumi e Yoshimura (1990) foram os pioneiros a realizarem uma análise de estabilidade global, via modos normais, de um escoamento laminar em um duto retangular. Estudos numéricos de Tatsumi e Yoshimura (1990) estabeleceram que o escoamento laminar em um duto retangular é estável a todas perturbações infinitesimais abaixo de uma relação de aspecto crítica de 3.2. Posteriormente Theofilis, Duck e Owen (2006) confirmaram o efeito estabilizante das paredes laterais por meio de análise linear, e mostraram que a teoria linear é incapaz de prever a instabilidade do escoamento em um duto retangular com uma relação de aspecto reduzida.

Um grande número de estudos têm se centrado sobre a origem do movimento secundário, que é observado na região dos cantos para as condições de turbulência (GAVRILAKIS, 1992; GALLETTI; BOTTARO, 2004). O processo de transição do estado laminar para turbulência nesta configuração tem recebido menos atenção.

Consideremos um duto retangular definido no domínio $\Omega=\{x \in[-A, A]\} \times\{y \in[-1,1]\}$ onde $A$ é a razão de aspecto. Um esquema da geometria para esse escoamento é apresentado na figura 4.4 .

O gradiente de pressão constante na direção $z$ leva a um escoamento laminar permanente que independe dessa direção e possui um vetor velocidade $(0,0, \bar{w})^{T}$. A componente 


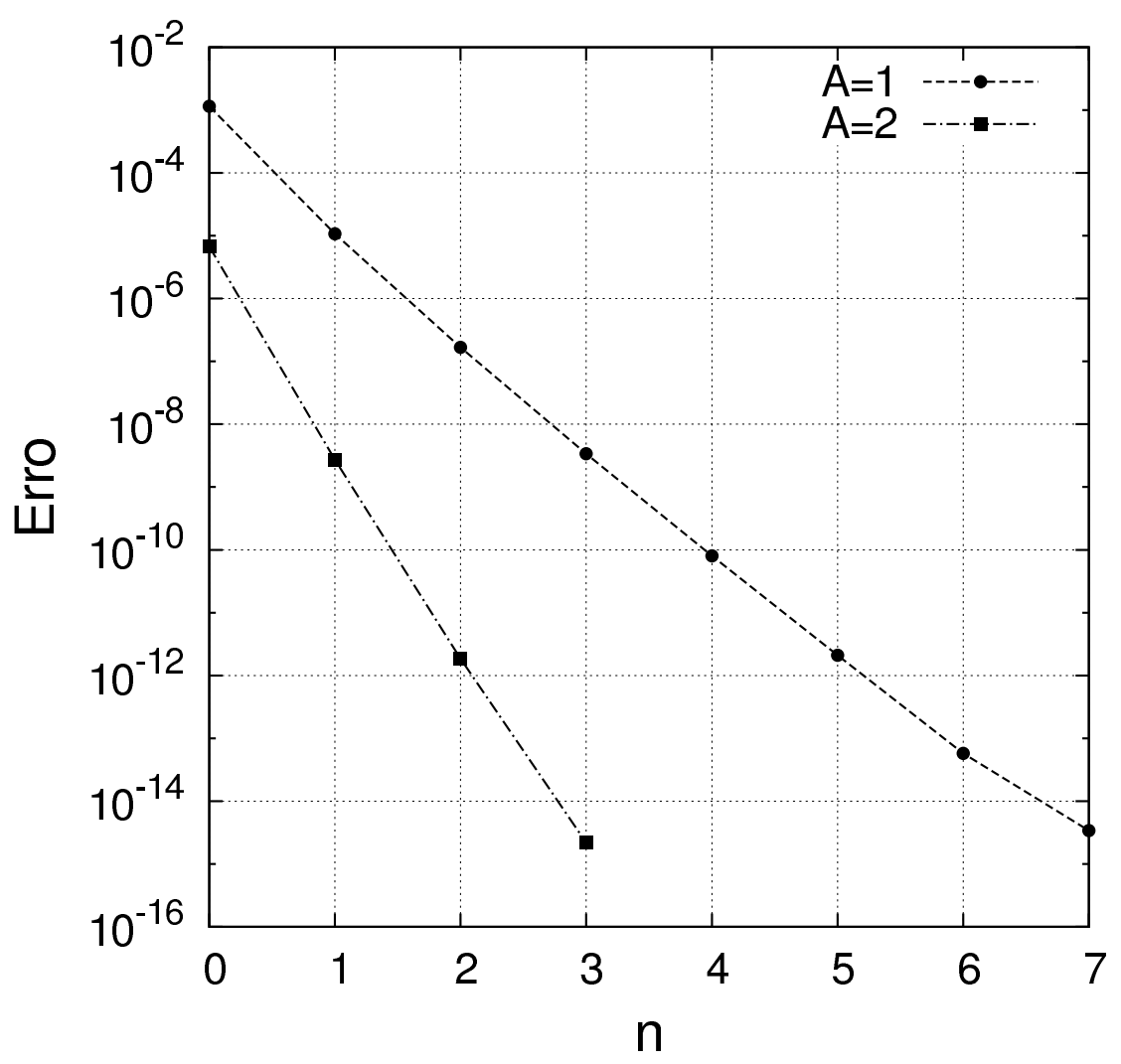

Figura 4.5: Erro de truncamento da solução analítica com respeito ao valor convergido $(A=1 \rightarrow n=8, A=2 \rightarrow n=4)$

de velocidade $\bar{w}(x, y)$ satisfaz a equação de Poisson

$$
\nabla^{2} \bar{w}(x, y)=\frac{\partial \bar{p}}{\partial z}=c
$$

onde $\nabla^{2}=\partial^{2} / \partial x^{2}+\partial^{2} / \partial y^{2}$ e $c$ é constante. As condições de contorno são

$$
\bar{w}(x, y=-1)=\bar{w}(y, x=-A)=\bar{w}(x, y=1)=\bar{w}(x=A, y)=0 .
$$

Tomando-se uma constante $c=2$ na Eq. (4.5), o problema de Poisson apresenta uma solução em forma de série (ROSENHEAD, 1963) dado por

$$
\bar{w}(x, y)=1-y^{2}-4\left(\frac{2}{\pi}\right)^{3} \sum_{n=0}^{\infty} \frac{(-1)^{n}}{(2 n+1)^{3}} \frac{\cosh [(2 n+1) \pi x / 2] \cos [(2 n+1) \pi y / 2]}{\cosh [(2 n+1) \pi A / 2]} .
$$

Adotando como valor de referência o valor máximo no domínio especificado, a figura 4.5 apresenta o erro de truncamento para essa série 4.7 para duas razões de aspecto para série com número crescentes de termos. Nota-se que com poucos termos obtém-se um erro 
de truncamento da ordem $O\left(10^{-15}\right)$. Além disso, observa-se que o aumento da razão de aspecto diminui o número de termos significativos da série, e a a equação 4.7 tende ao perfil de Poiseulle. O trabalho de Theofilis, Duck e Owen (2006) apresenta uma comparação entre as taxas de amplificação obtidas pela equação de Orr-Sommerfeld, para escoamento de Poiseulle, e uma análise de estabilidade global, adotando $4.7 \mathrm{com} A \gg 1$ como escoamento base. Esses resultados foram reproduzidos nesse trabalho e confirma-se boa concordância para os valores críticos descritos em (CRIMINALE; JACKSON; JOSLIN, 2003).

Em geral, são poucos os casos em que o escoamento base pode ser dado de maneira analítica. Dessa forma, a busca por uma solução numérica para a equação (4.5) pode ser interessante do ponto de vista de desenvolvimento e testes para o código numérico. Do ponto de vista numérico, casos como este em que uma comparação entre solução analítica e numérica, obtida por distintos métodos, pode ser realizada devem ser tomadas como ponto de partida para o desenvolvimento de um código e sua verificação. Assim, resolvendo numericamente a equação (4.5) para razão de aspecto unitário $(A=1)$, sujeito a condições de contorno do tipo Dirichlet homogêneas em $\partial \Omega$, a figura 4.6 apresenta o histórico de convergência.

Observa-se uma rápida convergência para ambos os métodos numéricos empregados, e um dos motivos é a estrutura matricial quase simétrica do problema, como apresentado nas figuras (3.6(a)) e (3.6(b)). Essa rápida convergência não pôde ser observada em outros problemas resolvidos neste trabalho.

Usando a teoria de análise da estabilidade global descrita na seção 2.2.4 e o escoamento básico descrito acima para $A=1$ adimensionalizado pelo valor máximo obtido no domínio físico, apresentamos breves resultados para o problema de autovalor Eq. (2.16) para número de Reynolds $R e=100$ e $\beta=1$. Os modos foram obtidos usando código incompressível bem como o código compressível. Para este último caso, o número de Mach adotado foi $10^{-3}$ e a densidade e temperatura iguais a 1 .

As condições de contorno empregadas no problema de autovalor foram: 


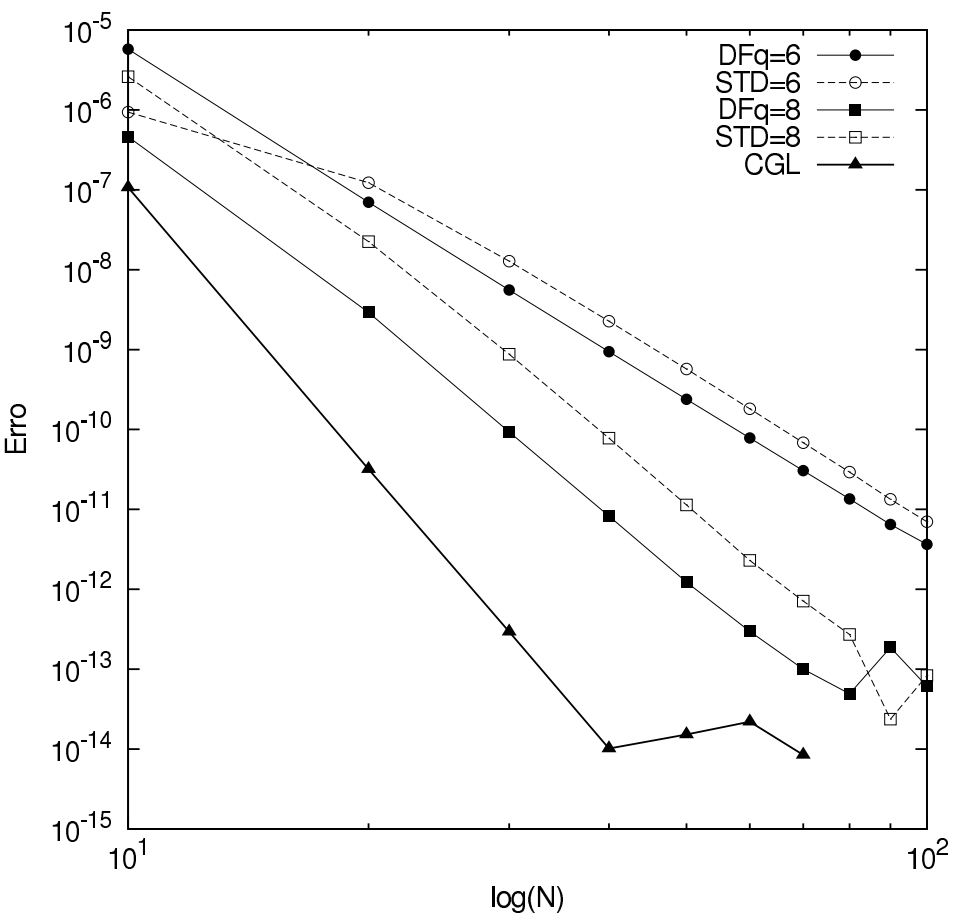

Figura 4.6: Histórico de convergência da solução numérica da equação de Poisson para razão de aspecto unitário $(A=1)$. O valor de $n=8$ foi assumido na Eq. (4.7), proposta por Rosenhead (1963), como valor referência.

- condição de contorno de Dirichlet homogênea para a componente de velocidade $w$ em $\partial \Omega$.

- condição de contorno de Neumann homogênea para pressão, i.e., $\partial_{n} p=0$ em $\partial \Omega$.

A autofunções correspondentes às três componentes de velocidade obtidas são apresentados na figura 4.7.
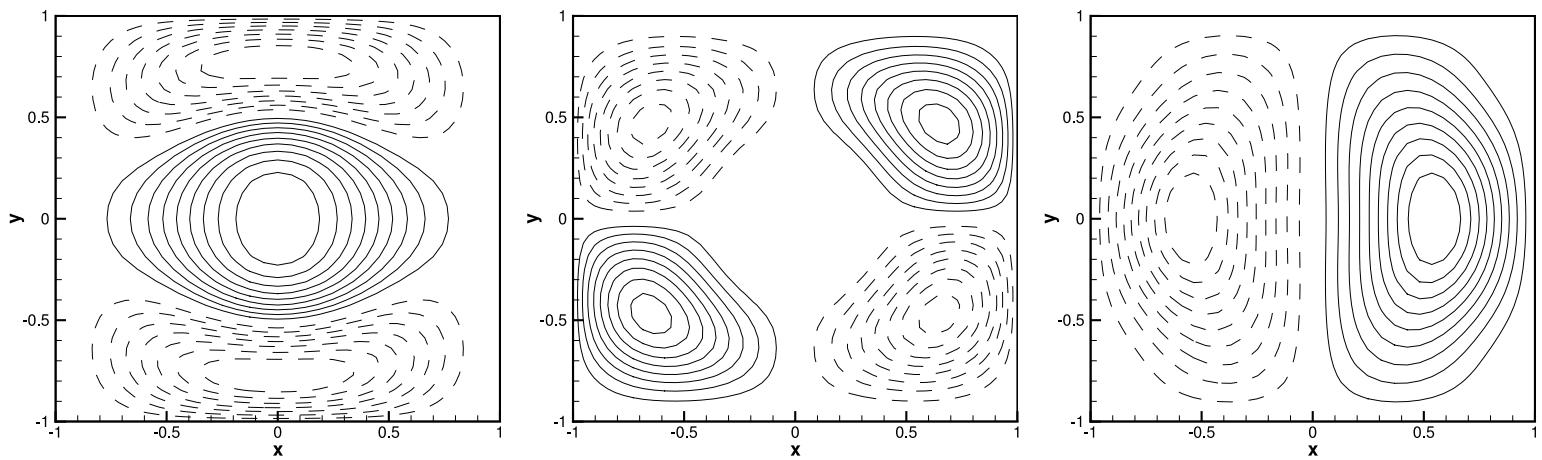

Figura 4.7: Autofunções, $u, v, w$, correspondente ao primeiro modo de um escoamento em um duto retangular, de razão de aspecto unitário.

Os dados de tempo e memória computacional obtidos no cálculo da taxa de amplifica- 
ção temporal do modo menos estável para o duto retangular de razão de aspecto unitária podem ser visto nas figuras 4.8 e 4.9. O histórico de convergência da taxa de amplificação pode ser vista na figura 4.10 para distintos métodos de dicretização utilizado. Mesmo com a alta acuracidade dos métodos espectrais, considerando a mesma faixa de erro relativo, os dados de memória e tempo computacional para os métodos de alta ordem de diferença finitas se apresenta mais vantajoso.

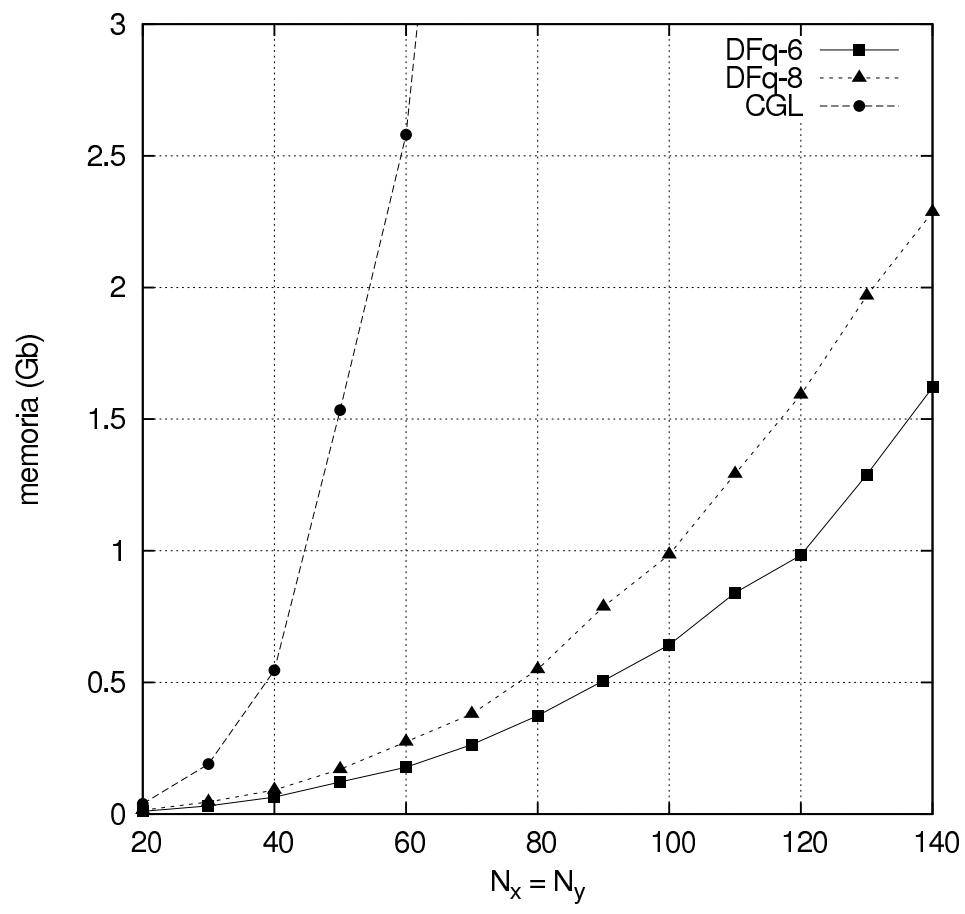

Figura 4.8: Memória computacional empregada na decomposição LU de um problema de autovalor para um escoamento em duto retangular de razão de aspecto unitário, $R e=100$ e $\beta=1$.

Destaca-se o alto custo computacional que os métodos espectrais empregados aqui apresentam e o ganho computacional que os métodos de diferenças finitas alta ordem apresentaram em relação aos métodos espectrais na resolução de problemas de instabilidade linear global. 


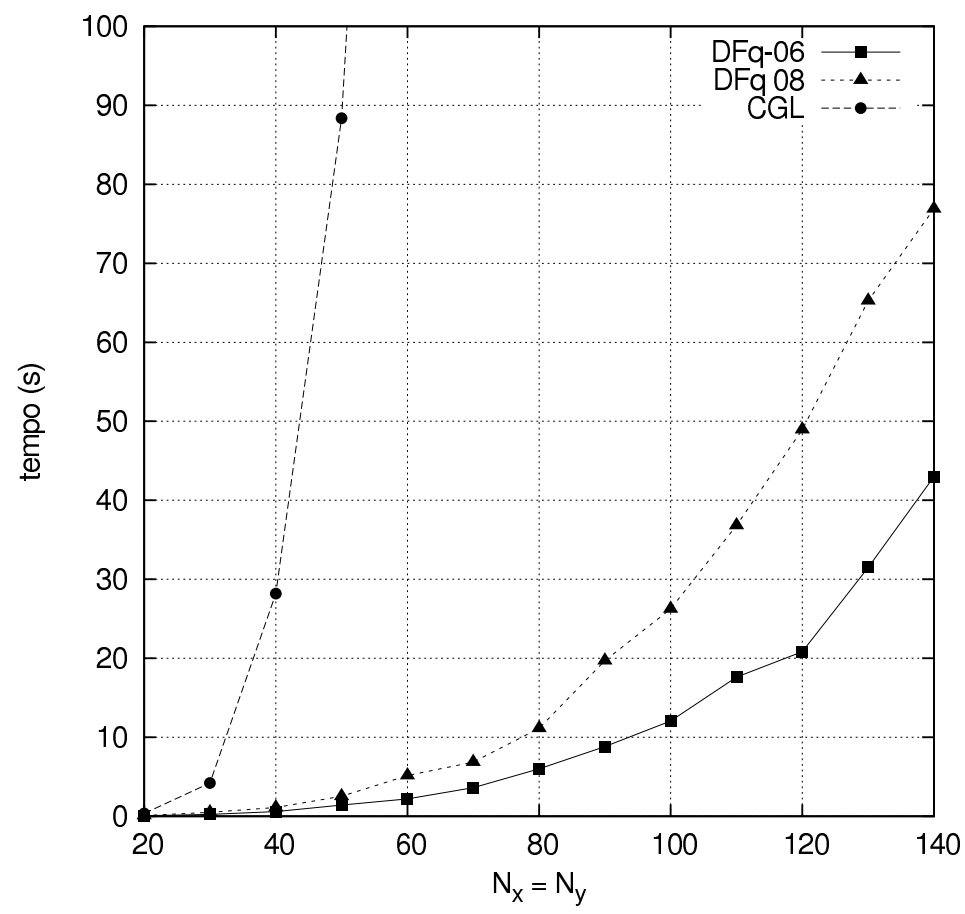

Figura 4.9: Tempo computacional empregado na decomposição LU de um problema de autovalor para um escoamento em duto retangular de razão de aspecto unitário, $R e=100$ e $\beta=1$.

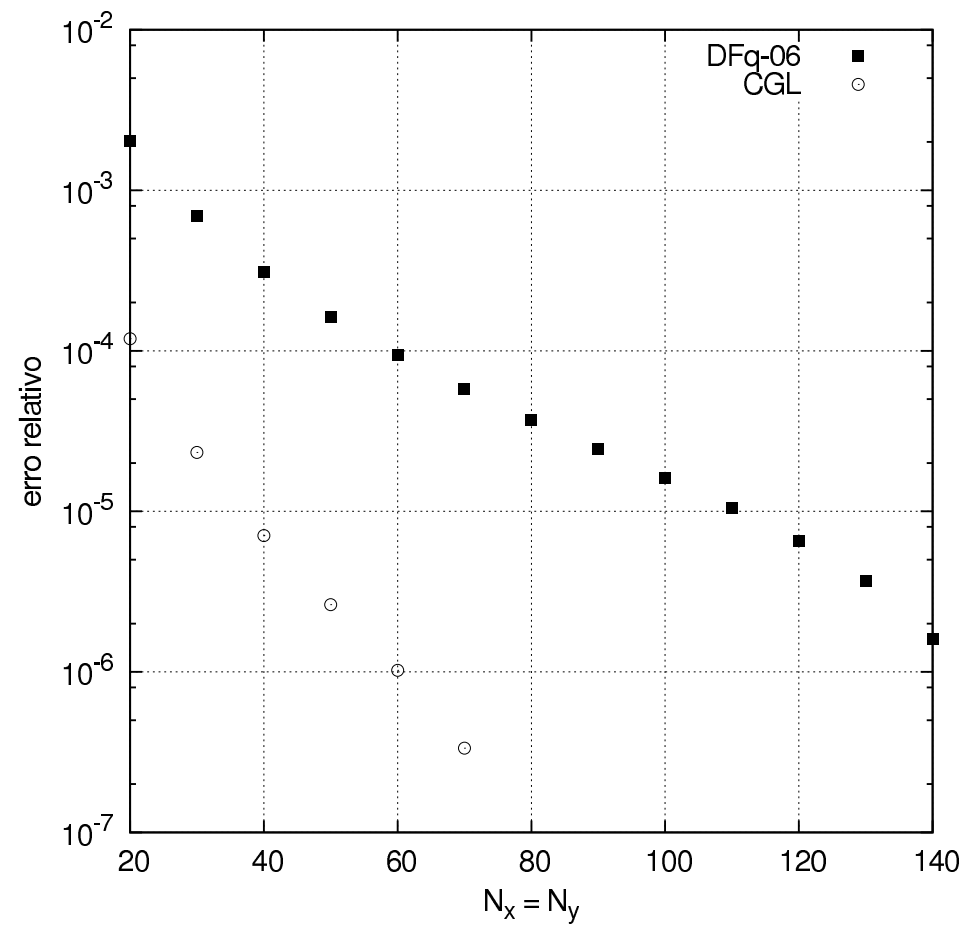

Figura 4.10: Erro relativo para o modo menos estável de um escoamento em um duto retangular de razão de aspecto unitário, $R e=100$ e $\beta=1$. 


\subsection{Instabilidade linear de um escoamento sobre uma cavidade com tampa deslizante}

O escoamento de uma cavidade com tampa deslizante é, provavelmente, um dos problemas mais estudos em mecânica dos fluidos computacional. A simplicidade da geometria torna o problema mais fácil de codificar e aplicar condições de contorno e etc. Mesmo que o problema parece simplificado, em muitos aspectos, o escoamento em uma cavidade mantém toda física de um escoamento com vórtices contra rotativos que aparecem nos cantos da cavidade, como pode ser visto na figura 4.11 que foi obtida a partir de uma simulações numéricas direta.

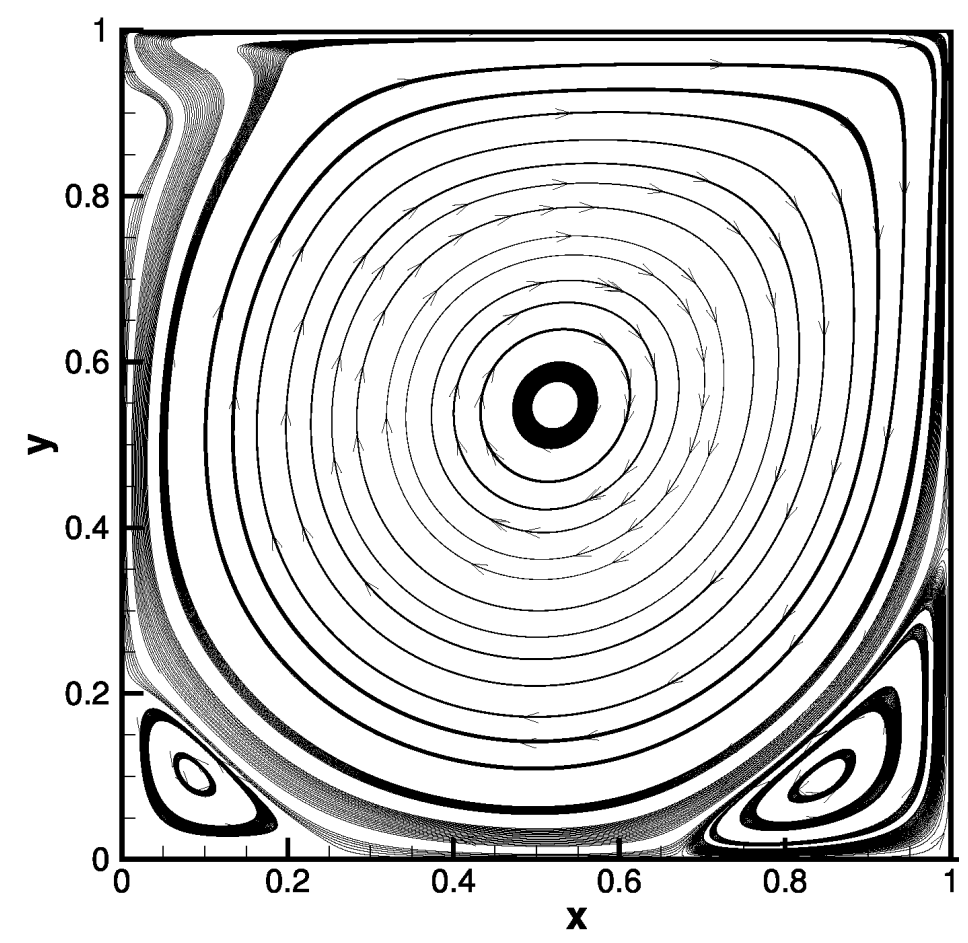

Figura 4.11: Linhas de corrente de uma cavidade fechada para $R \boldsymbol{e}=2000$.

O problema da cavidade fechada é o movimento de um fluido incompressível induzido por uma tampa que se movimenta tangencialmente a uma velocidade constante. Em um sistema extendido infinitamente na direção spanwise o escoamento é bidimensional para pequenos números de Reynolds. Por análise de estabilidade linear é mostrado que este escoamento base torna-se instável à números mais elevados de Reynolds para quatro modos tridimensionais e, é dependente da relação de aspecto da seção transversal. Para cavidade 
quadrada os resultados são confirmados por experimentos (SHANKAR; DESHPANDE, 2000).

Estudos em cavidade fechada com tampa deslizante é um excelente exemplo de previsões bem sucedidas de análise global. Considerando a falta de homogeneidade nas direções espaciais de um escoamento 2D com tampa deslizante, o uso da análise global linear torna-se essencial.

Escoamentos de uma cavidade fechada com tampa deslizante foi revisto por (SHANKAR; DESHPANDE, 2000), que forneceu uma descrição dos fenômenos deste escoamento em duas e três dimenssões espaciais. Praticamente todo trabalho de análise de instabilidade global foi realizada após esta revisão, dessa forma, o papel dos modos próprios globais para descrever a instabilidade e tridimensionalidade nesta classe de escoamento é bem compreendida.

Vários autores tem pesquisado por bifurcação de Hopf no escoamento de cavidade fechada resolvendo problemas de autovalor bi-dimensional onde o número de onda na direção spanwise, $\beta=2 \pi / L z=0$, é considerado. O valor crítico do escoamento bi-dimensional é considerado por muitos como sendo $R e \approx 8000$. Entretanto, segundo Theofilis, Duck e Owen (2006) essa discussão é meramente acadêmica tendo em vista que os escoamentos tri-dimensionais tem o valor de Reynolds crítico inferior à $R e_{2 D} \approx 8000$.

Simulações numéricas diretas foram realizadas para obter o escoamento base. Para isso, foram utilizados 2 códigos computacionais, a saber, Semtex ${ }^{1}$ e o código utilizado no trabalho de Theofilis, Duck e Owen (2006).

A figura 4.12 apresenta o escoamento base para um escoamento em uma cavidade fechada com tampa deslizante para número de Reynolds $R \boldsymbol{e}=582$.

Para o movimento da tampa deveria ser imposto $u=1$ em $y=1$ para todo $x$, entretanto, instabilidades numéricas podem surgir próximo aos pontos $(0,1)$ e $(1,1)$. Dessa forma, seguindo a literatura, a condição de contorno para a componente de velocidade $u$

\footnotetext{
${ }^{1}$ http://users.monash.edu.au/ bburn/semtex.html
} 


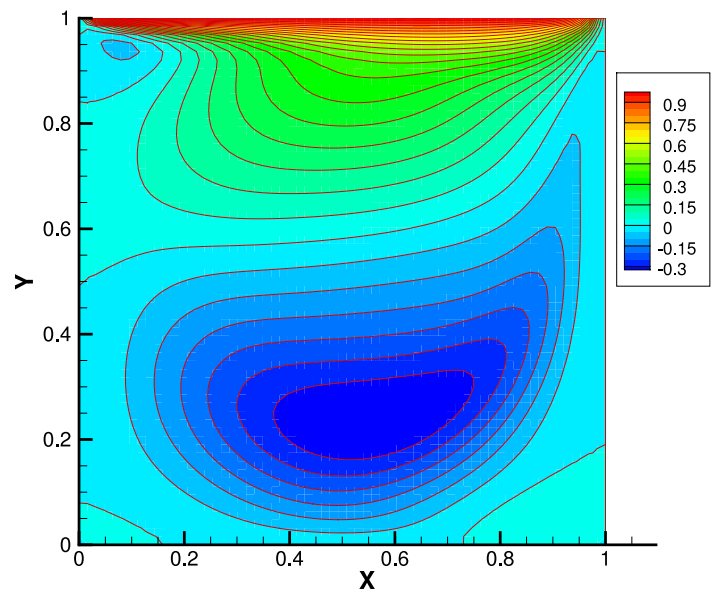

(a) $\mathrm{u}$

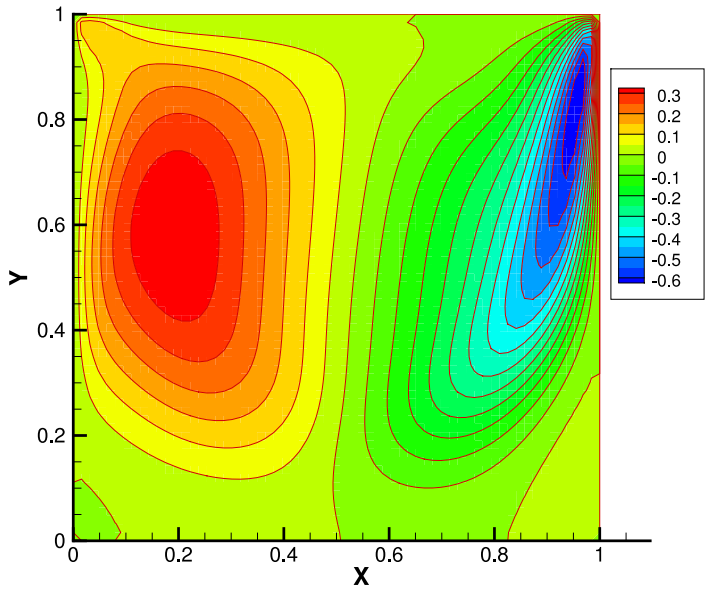

(b) $\mathrm{v}$

Figura 4.12: Componentes de velocidade de uma cavidade fechada regularizada para $R e=582$.

em $y=1$ é dado por:

$$
u=\left(1.0-(2.0 x-1.0)^{18}\right)^{2} .
$$

Uma cavidade com essa condição de contorno diferente de $u$ constante chamamos de cavidade regularizada. Para os contornos, foram utilizados condição de Dirichlet homogêneas para as componentes de velocidade $u$ e $v$ e Neumann para a pressão em todos os contornos do domínio.

Resolvemos o problema de autovalor 2.16 para o problema da cavidade fechada, com tampa deslizante, onde o escoamento base adotado foi apresentado na figura 4.12, utilizando dois códigos computacionais: versão compressível considerando $M a=10^{-4}$ incompressível como método de verificação.

A figura 4.13 apresenta os autovalores obtidos ${ }^{2}$ pelas duas versões dos códigos. Observase uma excelente concordância entre os espectros, incompressível e compressível, onde o número de Mach foi pequeno para o caso em que o código compressível foi utilizado. Nota-se uma diferença entre os espectros modos que diferentes dos fundamentais e isso ocorre devido aos modos acústicos que um escoamento compressível possui.

\footnotetext{
${ }^{2}$ Foi utilizado a mesma dimensão do subespaço de Krylov para método de Arnoldi.
} 


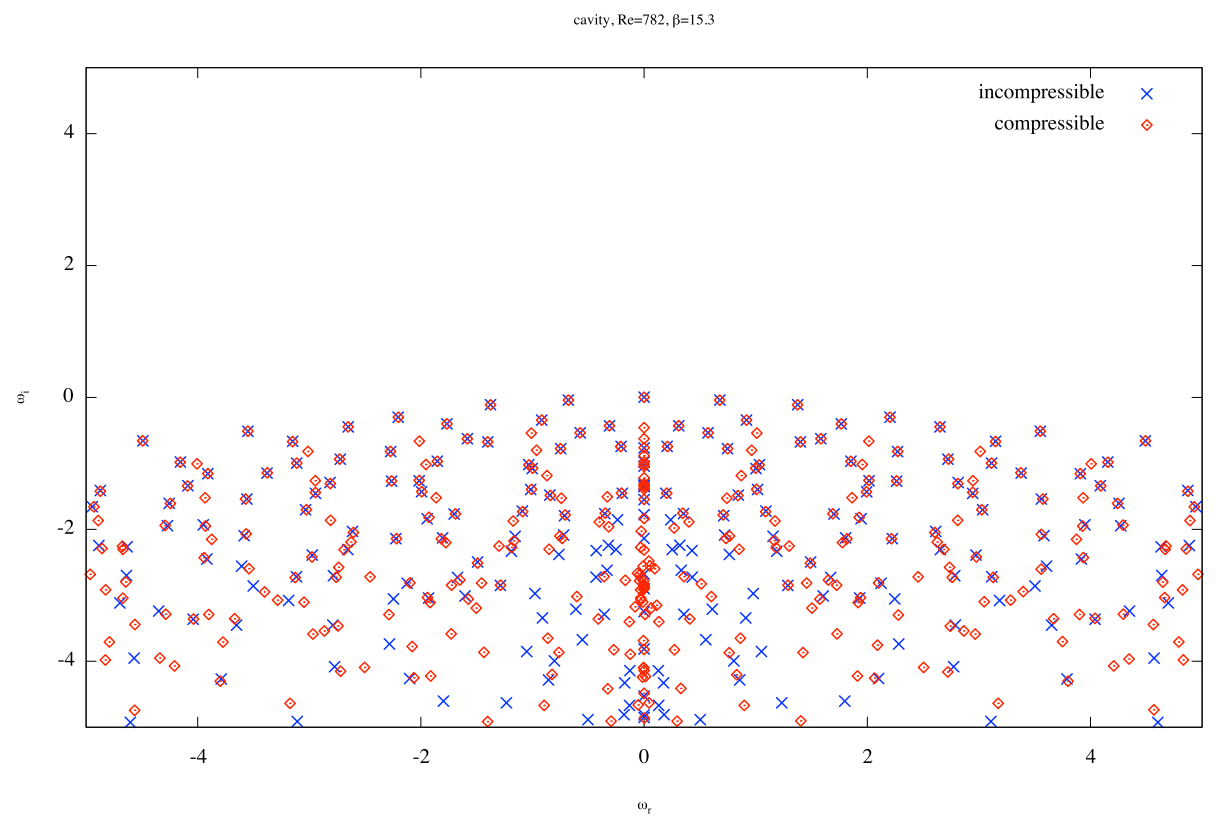

Figura 4.13: Espectro completo obtido para o problema da cavidade fechada $R e=782$ e $\beta=15.3$.

O problema da cavidade fechada foi utilizado neste trabalho no contexto de verificação do código computacional desenvolvido. 


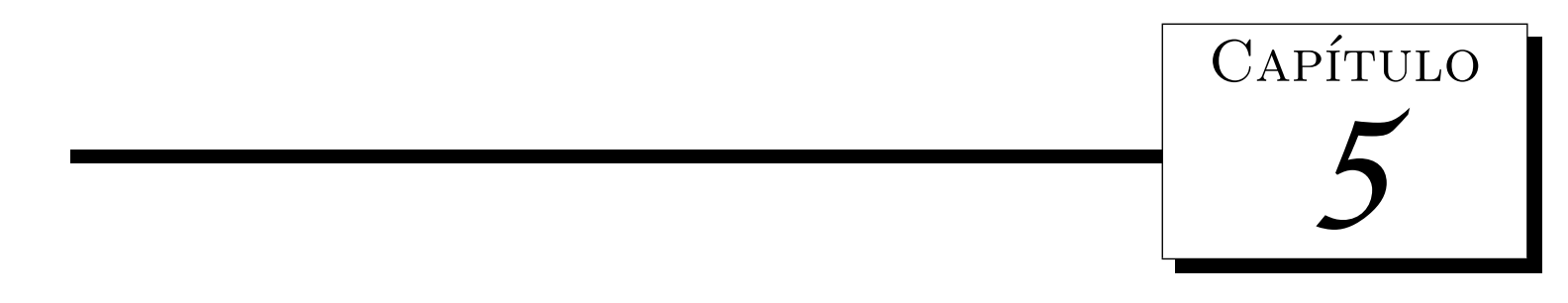

\section{Camada limite de uma linha de estagnação de}

\section{uma asa}

A instabilidade do escoamento próximo ao bordo de ataque da asa de uma aeronave tem um grande significado sobre o aspecto de engenharia. Instabilidades deste tipo podem promover o crescimento de perturbações que serão convectadas a jusante, tendo influência direta sobre o processo de transição de escoamento laminar para turbulento na superfície da asa. Estudos sobre os mecanismos de instabilidades neste escoamento podem fornecer informações úteis no projeto aerodinâmico de asas.

Um escoamento que incide sobre uma superfície plana e sólida em uma direção arbitrária, como pode ser visto esquematicamente na figura (5.1), ou ainda, uma representação bidimensional dos vetores velocidade deste escoamento apresentado na figura 5.2, é uma simplificação natural deste escoamento que pode ser analisado segundo a teoria de estabilidade global. Do ponto de vista numérico, o estudo de instabilidade desse escoamento é apresentado aqui a fim de avaliar o desepenho do algoritmo discutido neste trabalho, uma vez que a presença de três componentes de velocidade do escoamento básico constituem um caso teste bastante desafiador para um código que realiza análise de instabilidade global.

Análise da instabilidade linear de um escoamento de um ponto de estagnação ortogonal 


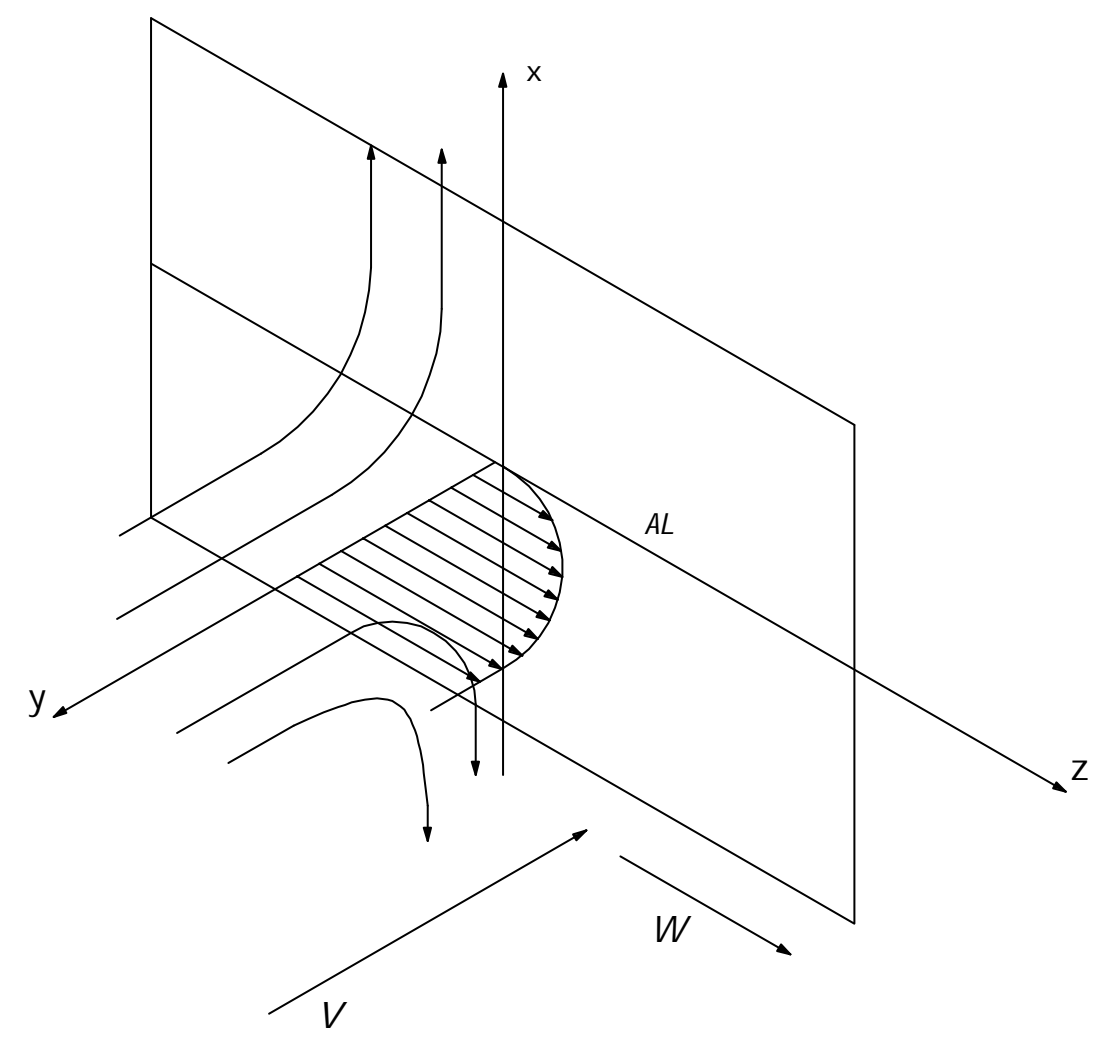

Figura 5.1: Representação esquemática dos vetores velocidade do escoamento externo.

foi primeiramente realizado por Görtler (1955) e Hämmerlin (1955) no regime incompressível. Fizeram isto através de uma redução das equações governantes de Navier-Stokes linearizadas em um sistema de equações diferenciais ordinárias que incorpora uma simetria do escoamento básico proposto por Hiemenz (1911). Mais tarte, Hall, Malik e Poll (1984) analisaram um escoamento ortogonal sobre uma linha de estagnação através de um problema de autovalor e Theofilis (1993) como um problema de valor inicial, ambos em contexto temporal, enquanto Theofilis (1995) resolveu o problema de autovalor espacial deste problema.

Usando um modelo de similaridade para as perturbações, Hall, Malik e Poll (1984) foram os primeiros à analisar a instabilidade linear deste escoamento, em regime incompressível, adotando o modelo de escoamento básico de Görtler (1955) e Hämmerlin (1955).

Lin e Malik (1996) é o primeiro trabalho que faz uma análise de instabilidade global de um escoamento sobre uma linha de estagnação ortogonal, nas proximidades da linha de recolamento, resolvendo um problema de autovalor baseado nas equações diferenciais 


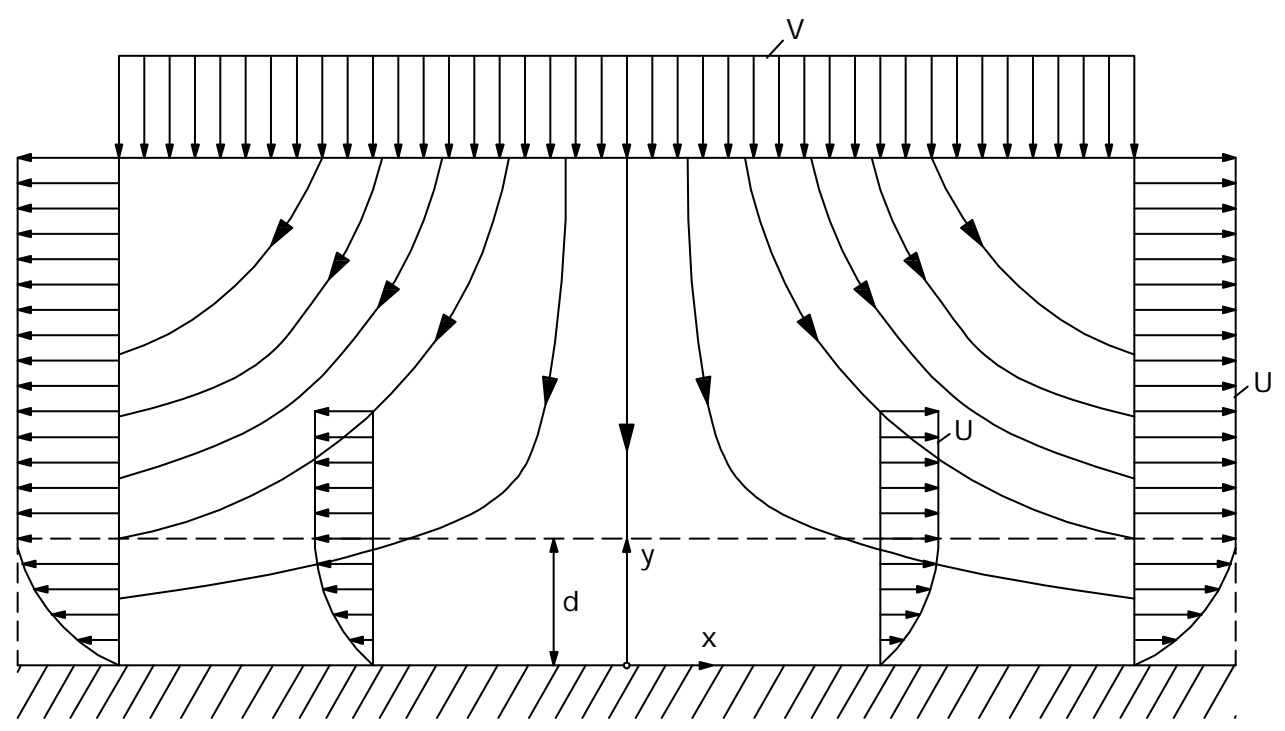

Figura 5.2: Representação esquemática 2D dos vetores velocidade do escoamento.

parciais e uma representação bi-dimensional para as perturbações em torno do escoamento de Hiemenz. Além disso, recuperaram os resultados anteriores para o autovalor mais instável. Eles passaram a incluir a curvatura e concluiram que seu efeito é estabilizante (LIN; MALIK, 1997).

A primeira solução para este problema em regime compressível foi dado por Theofilis, Fedorov e Collis (2004) e mais tarde, complementada pelo trabalho Gennaro et al. (2011b). Os resultados de Theofilis, Fedorov e Collis (2004) para análise de instabilidade linear biglobal compressível foram obtidos resolvendo um problema de autovalor denso. Além disso, apresentam uma teoria assintótica e um modelo polinomial para o escoamento de Hiemenz incompressível na mesma linha do trabalho de Theofilis et al. (2003), revisto por Theofilis (2003). O modelo polinomial pode ser estendido para o escoamento compressível para a faixa de número de Mach subsônica.

Contribuições de Obrist e Schmid (2003) forneceram uma descrição alternativa do espectro de autovalores deste escoamento, com base em polinômios de Hermite ${ }^{1}$. A primeira análise não-modal deste escoamento foi também realizada por estes autores, que demonstraram o papel desempenhado pelo espectro contínuo no crescimento do transiente. As

\footnotetext{
${ }^{1}$ Conjunto de polinômios ortogonais sobre um domínio $(-\infty, \infty)$ com função de ponderação $e^{\left(-x^{2}\right)}$
} 
equações adjuntas do problema BiGlobal foram derivadas e usadas para realizar estudos de receptividade e mostraram a conversão de alguns modos em modos globais dominante.

Instabilidade na vizinhaça de um cilindro unitário em um escoamento em regime compressível foi recentemente abordado por Duc, Sesterhenn e Friedrich (2006), Mack, Schmid e Sesterhenn (2008), Mack e Schmid (2010b), Mack e Schmid (2010a), Mack e Schmid (2011) e destaca-se a família de modos (hidrodinâmica ou acústica) identificadas de diferentes origens físicas. Ao contrário destas últimas obras, em que a computação em larga escala tem sido utilizada para estabelecer um escoamento base e estudar o desenvolvimento da instabilidade posteriormente, a contribuição deste trabalho mantém o modelo de escoamento básico para a camada limite e explora as abordagens computacionais aqui desenvolvidas concluindo o estudo paramétrico iniciado por Theofilis, Fedorov e Collis (2004).

A Seção (5.1) apresenta o escoamento básico analisado e a Seção (5.2) alguns aspectos numéricos envolvidos na solução do problema. Uma pequena discussão e testes para o caso incompressível e os principais resultados obtidos estão na seção (5.3). Neste trabalho, o foco de estudo está na vizinhaça de uma linha de estagnação de um bordo de ataque de uma asa onde desconsidera-se a curvatura, facilitando o estudo paramétrico.

\subsection{Escoamento Base}

O escoamento nas proximidades do bordo de ataque de uma asa foi aproximado neste trabalho como um escoamento de uma linha de estagnação do bordo de ataque de uma asa com velocidade do escoamento livre constante ao longo dela. Aqui, o escoamento é considerado compressível, como foi feito por Theofilis, Fedorov e Collis (2004), ao contrário de trabalho anteriores Hall, Malik e Poll (1984), Lin e Malik (1996), Theofilis et al. (2003) nas quais o escoamento é considerado incompressível.

Neste trabalho, $x$ e $y$ são as coordenadas tangencial (longitudinal) e normal, $z$ a coordenada ao longo da linha de estagnação e suas respectivas componentes de velocidades 
dadas por $U, V$ e $W$. Uma solução de similaridade foi proposta por Cohen e Reshotko (1956) (ver também (MACK, 1984)), para que o escoamento no plano $x$ - $y$ seja obtido na forma de uma função de corrente bi-dimensional. Se $v$ é a viscosidade cinemática e $S$ a taxa de deformação local definida por $S=\left(d U_{e} / d x\right)_{x=0}$ (onde o subscrito $e$ refere-se à borda da camada limite), uma escala de referência para comprimento pode ser definida como $\Delta=(v / S)^{1 / 2}$. As variáveis de similaridade são definidas como $x / \Delta$ e $y / \Delta$, respectivamente. A função de corrente para solução de similaridade tem a forma

$$
\psi(\xi, \eta)=v \xi f(\eta)
$$

Assume-se que a componente de velocidade transversal depende somente da direção normal $\eta$. Os números de Mach e Reynolds são definidos usando-se a velocidade $W_{e}$, a velocidade do som $a_{e}$ e a viscosidade ${ }^{2}$ cinemática $v_{e}$, no escoamento livre e da taxa de deformação:

$$
M a=W_{e} / a_{e}, R e=W_{e} \Delta / v_{e}
$$

o número de Prandtl foi tomado como 0.72.

As equações da camada limite laminar compressível independem do número de Reynolds e podem ser obtidas usando-se a transformação de Illingworth-Stewartson definida por:

$$
X=\int_{0}^{x} \lambda \frac{p_{e} c_{e}}{p_{0} c_{0}} d x, \quad Y=\frac{a_{e}}{a_{0}} \int_{0}^{y} \lambda \frac{\rho}{\rho_{0}} d y
$$

onde $p$ é a pressão e o subscrito $o$ indica os valores do escoamento livre.

Considerando-se as equações (5.1) e (5.3), os perfis de velocidade e temperatura podem ser obtidos como solução do seguinte conjunto de equações diferenciais ordinárias

\footnotetext{
${ }^{2}$ Uma dependência da viscosidade com a temperatura está permitida neste modelo.
} 
(COHEN; RESHOTKO, 1956; MACK, 1984), e são elas:

$$
\begin{aligned}
V^{\prime} & =-U+V \frac{T^{\prime}}{T} \\
U^{\prime \prime} & =\frac{1}{\mu}\left(\frac{U^{2}+V U^{\prime}}{T}-1-\frac{\partial \mu}{\partial T} T^{\prime} U^{\prime}\right) \\
W^{\prime \prime} & =\frac{1}{\mu}\left(\frac{V W^{\prime}}{T}-\frac{\partial \mu}{\partial T} T^{\prime} W^{\prime}\right) \\
T^{\prime \prime} & =\frac{P r}{\mu}\left(-\frac{\partial \mu}{\partial T} \frac{T^{\prime 2}}{P r}+\frac{T^{\prime} V}{T}-(\gamma-1) M a^{2} \mu W^{\prime 2}\right)
\end{aligned}
$$

complementado com as seguintes condições de contorno:

$$
\begin{aligned}
& U(0)=W(0)=0, V(0)=0 \\
& U(\infty)=W(\infty)=T(\infty)=1 .
\end{aligned}
$$

O sistema (5.4-5.9) foi resolvido usando um "shooting method" e os perfis de velocidade e temperatura podem ser vistos na figura 5.3. Além disso, as linhas de corrente sobrepostas ao campo de velocidade de $u$ podem ser vistas na figura (5.4). A tabela (5.1) apresenta valores de temperatura e deformação-cisalhamento na parede para diferentes números de Mach. Uma tabela contendo valores intermediários pode ser vista na seção 7.3.

Tabela 5.1: Dependência de $U_{0}{ }^{\prime}(0), W_{0}{ }^{\prime}(0)$ e temperatura na parede do escoamento base em vários $M a$.

\begin{tabular}{c|ccc}
\hline$M a$ & $U_{0}{ }^{\prime}(0)$ & $W_{0}{ }^{\prime}(0)$ & $T_{0}(0)$ \\
\hline 0.02 & 1.232561 & 0.570437 & 1.000069 \\
0.25 & 1.228483 & 0.566095 & 1.010746 \\
0.50 & 1.216689 & 0.553511 & 1.042990 \\
0.75 & 1.198610 & 0.534132 & 1.096741 \\
0.90 & 1.185527 & 0.520035 & 1.139322 \\
1.00 & 1.176141 & 0.509881 & 1.172016 \\
1.50 & 1.125582 & 0.454480 & 1.387198 \\
2.00 & 1.076630 & 0.399360 & 1.688629 \\
3.00 & 1.000514 & 0.308888 & 2.550336 \\
4.00 & 0.952139 & 0.245969 & 3.757095 \\
5.00 & 0.921755 & 0.202362 & 5.308874 \\
\hline
\end{tabular}



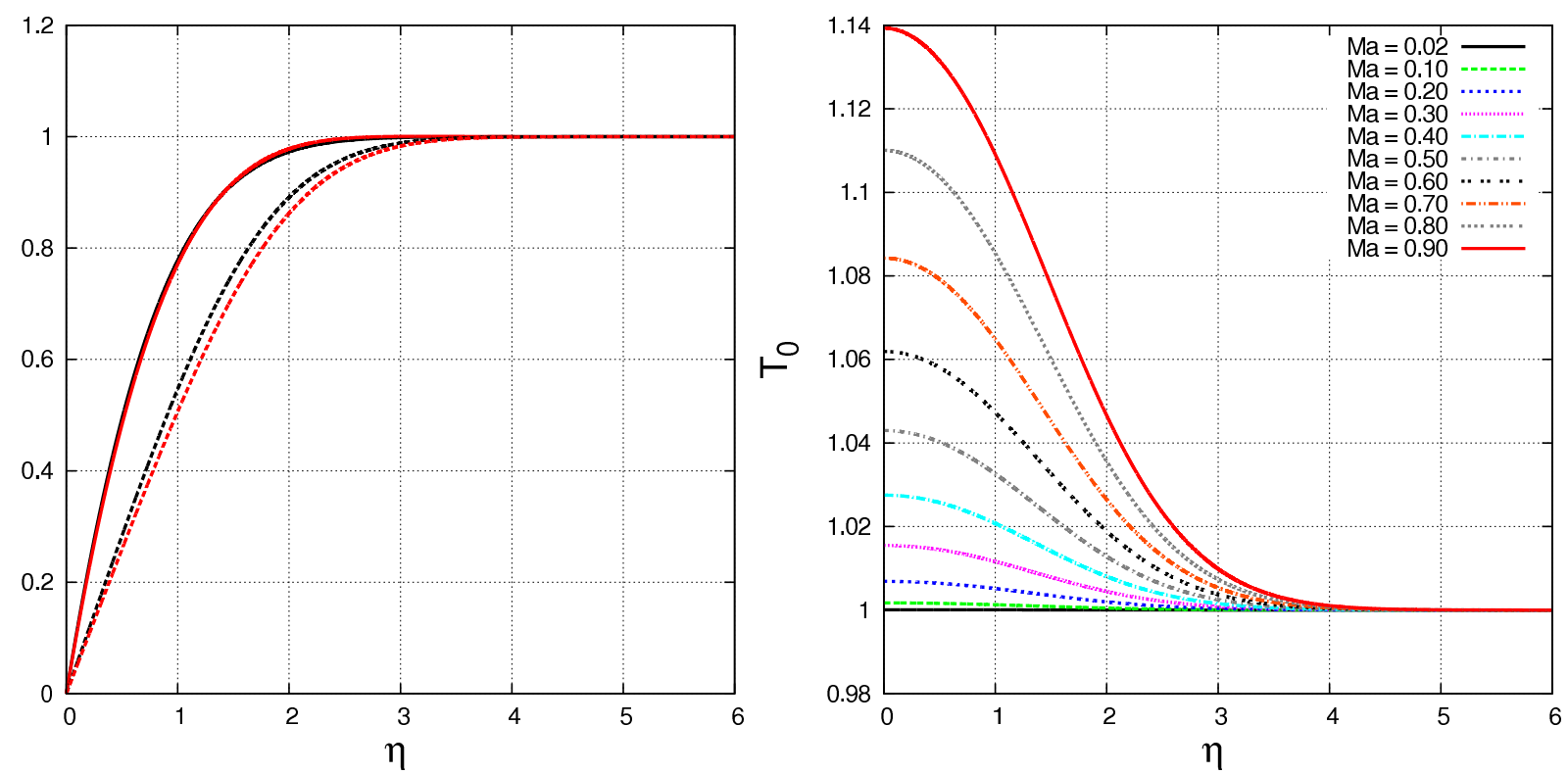

Figura 5.3: Solução do escoamento base. (a) componentes de velocidade longitudinal (linhas sólidas) e transversal (linhas tracejadas) para números de Mach $M a=0.02$ (cor preta) e $M a=0.90$ (cor vermelha). (b) perfis de temperatura para diferentes números de Mach.

\subsection{Análise do Desempenho do Algorítmo Implemen- tado}

Nesta seção, apresentaremos análise do desempenho do algorítmo implementado para a solução numérica do problema de autovalor global de uma camada limite de uma linha de estagnação. Conforme descrito no capítulo $3 \mathrm{um}$ novo algoritmo foi proposto e implementado para a realização deste trabalho.

As equações de continuidade, Navier-Stokes e energia foram linearizadas, como apresentado na seção (2.2.4), sobre o escoamento base apresentado na seção (5.1) e a solução numérica do problema de autovalor (2.16) foi então obtida considerando-se o seguinte conjunto de condições de contorno:

- na parede, uma condição de não-deslizamento foi imposta e ao longo dela, $\frac{\partial p}{\partial y}=0$;

- um decaimento rápido foi assumido para as perturbações na direção normal e uma condição de contorno do tipo Dirichlet foi imposta à todas variáveis no campo distante; 


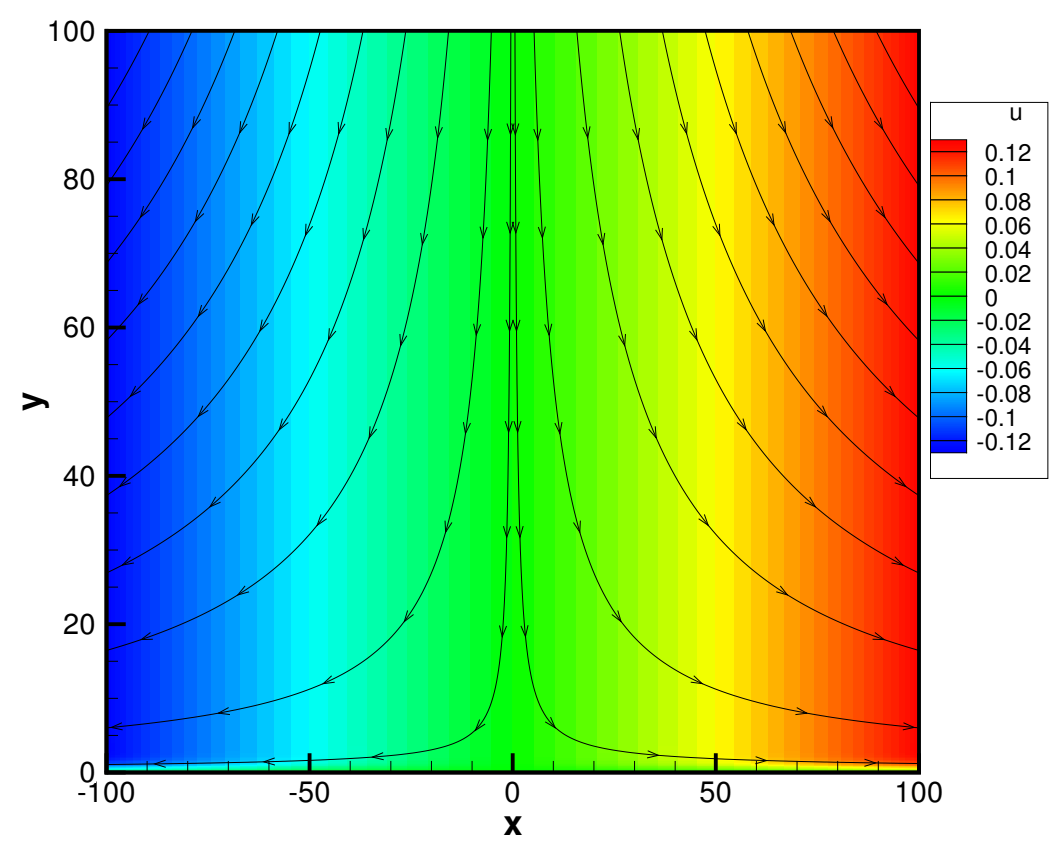

Figura 5.4: Linhas de corrente sobrepostas ao campo de velocidade $u$ em $M a=0.90$

- extrapolação linear dos pontos do interior do domínio computacional foi usada para todas variáveis em $x= \pm L_{x}$.

O desempenho do código foi analisado sob vários aspectos, entre eles, a performance dos métodos de redução dos coeficientes de preenchimento, memória e tempo computacional.

A respeito do desempenho dos diferentes métodos para redução dos coeficientes de preenchimento que foram discutidos na seção (3.7), observou-se a quantidade de elementos não-nulos produzidos pelos diferentes métodos de reordenação dos elementos. Os resultados foram compilados em tabelas para os casos incompressível e compressível considerando o diferentes métodos de discretização utilizados neste trabalho que foram:

- discretização em pontos de colocação Chebyshev-Gaus-Lobatto em ambas direções $x$ e $y$ (CGL-CGL),

- discretização em diferenças finitas padrão e otimizadas em ambas direções $x$ e $y$ (DFq - DFq), 
- discretização em pontos de colocação Chebyshev-Gaus-Lobatto na direção $x$ e diferenças finitas padrão e otimizadas na direção y (CGL - DFq),

- discretização em diferenças finitas padrão e otimizadas na direção $x$ e pontos de colocação Chebyshev-Gaus-Lobatto na direção y (DFq - CGL).

Distintos métodos foram testados com objetivo de identificar o melhor método, ou ainda, o mais adequado para o estudo de estabilidade global de escoamentos compressíveis. Com uma certa frequência existe uma necessidade de realizar um estudo paramétrico, o que representa uma quantidade enorme de simulações computacionais a serem realizadas.

A tabela (5.2) apresenta os resultados para o número de elementos não-nulos, produzidos pelos diferentes métodos de reordenação dos elementos, para escoamento em regime incompressível e compressível. Os dados se referem a discretização espacial em pontos de colocação de Gauss-Lobatto em ambas direções. A tabela (5.3) apresenta também os resultados para o número de elementos não-nulos para escoamento compressível, entretanto, uma discretização espacial por diferenças finitas em ambas direções foi considerada. Os resultados dos coeficientes de preenchimento para uma combinação destes dois métodos de discretizações em cada direção pode ser visto na tabela (5.4). 
Tabela 5.2: Step I: Resultado dos coeficientes de preenchimento como função da resolução da malha computacional, para solução numérica do problema de autovalor BiGlobal discretizado em pontos de colocação espectral em ambas direções, usando diferentes métodos de ordenação

\section{CGL-CGL}

\begin{tabular}{cccccc}
\hline \multicolumn{7}{c}{ Incompressível } \\
\hline Grid & não nulos & AMD & AMF & PORD & METIS \\
\hline $11 \times 11$ & $1.10 \mathrm{E}+4$ & $1.05 \mathrm{E}+5$ & $1.04 \mathrm{E}+5$ & $1.16 \mathrm{E}+5$ & $1.03 \mathrm{E}+5$ \\
$21 \times 21$ & $8.38 \mathrm{E}+4$ & $1.63 \mathrm{E}+6$ & $1.61 \mathrm{E}+6$ & $1.74 \mathrm{E}+6$ & $1.57 \mathrm{E}+6$ \\
$31 \times 31$ & $2.78 \mathrm{E}+5$ & $8.11 \mathrm{E}+6$ & $8.00 \mathrm{E}+6$ & $8.59 \mathrm{E}+6$ & $7.25 \mathrm{E}+6$ \\
$41 \times 41$ & $6.55 \mathrm{E}+5$ & $2.58 \mathrm{E}+7$ & $2.56 \mathrm{E}+7$ & $2.72 \mathrm{E}+7$ & $2.52 \mathrm{E}+7$ \\
$51 \times 51$ & $1.27 \mathrm{E}+6$ & $6.22 \mathrm{E}+7$ & $6.32 \mathrm{E}+7$ & $6.63 \mathrm{E}+7$ & $5.89 \mathrm{E}+7$ \\
$61 \times 61$ & $2.19 \mathrm{E}+6$ & $1.30 \mathrm{E}+8$ & $1.28 \mathrm{E}+8$ & $1.38 \mathrm{E}+8$ & $1.19 \mathrm{E}+8$ \\
$71 \times 71$ & $3.48 \mathrm{E}+6$ & $2.32 \mathrm{E}+8$ & $2.39 \mathrm{E}+8$ & $2.55 \mathrm{E}+8$ & $2.42 \mathrm{E}+8$ \\
\hline
\end{tabular}

\begin{tabular}{cccccc}
\hline \multicolumn{6}{c}{ Compressível } \\
\hline Grid & não nulos & AMD & AMF & PORD & METIS \\
\hline $11 \times 11$ & $4.71 \mathrm{E}+4$ & $2.35 \mathrm{E}+5$ & $2.46 \mathrm{E}+5$ & $2.64 \mathrm{E}+5$ & $2.26 \mathrm{E}+5$ \\
$21 \times 21$ & $5.49 \mathrm{E}+5$ & $3.60 \mathrm{E}+6$ & $3.63 \mathrm{E}+6$ & $3.75 \mathrm{E}+6$ & $3.17 \mathrm{E}+6$ \\
$31 \times 31$ & $2.40 \mathrm{E}+6$ & $1.81 \mathrm{E}+7$ & $1.80 \mathrm{E}+7$ & $1.99 \mathrm{E}+7$ & $1.59 \mathrm{E}+7$ \\
$41 \times 41$ & $6.99 \mathrm{E}+6$ & $5.61 \mathrm{E}+7$ & $5.64 \mathrm{E}+7$ & $6.30 \mathrm{E}+7$ & $5.26 \mathrm{E}+7$ \\
$51 \times 51$ & $1.62 \mathrm{E}+7$ & $1.38 \mathrm{E}+8$ & $1.39 \mathrm{E}+8$ & $1.52 \mathrm{E}+8$ & $1.24 \mathrm{E}+8$ \\
$61 \times 61$ & $3.23 \mathrm{E}+7$ & $2.76 \mathrm{E}+8$ & $2.92 \mathrm{E}+8$ & $3.16 \mathrm{E}+8$ & $2.59 \mathrm{E}+8$ \\
$71 \times 71$ & $5.81 \mathrm{E}+7$ & $5.14 \mathrm{E}+8$ & $5.37 \mathrm{E}+8$ & $6.02 \mathrm{E}+8$ & $4.65 \mathrm{E}+8$ \\
\hline
\end{tabular}




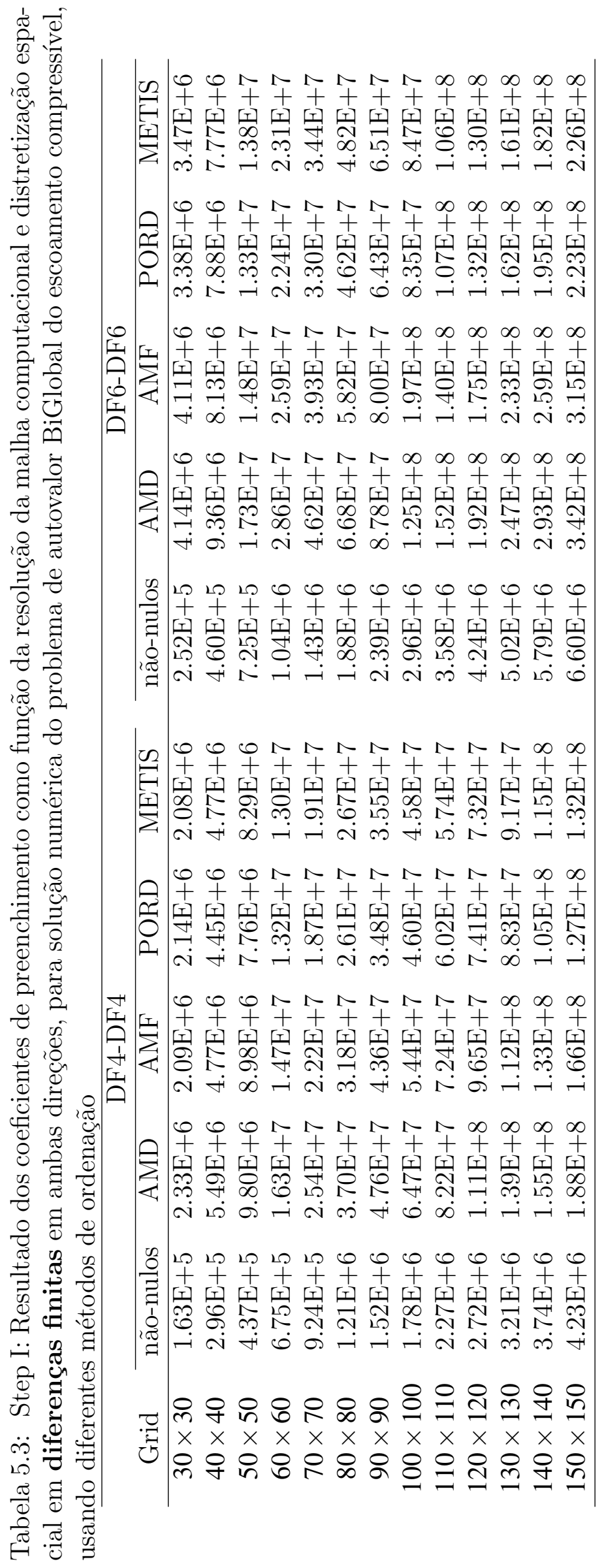




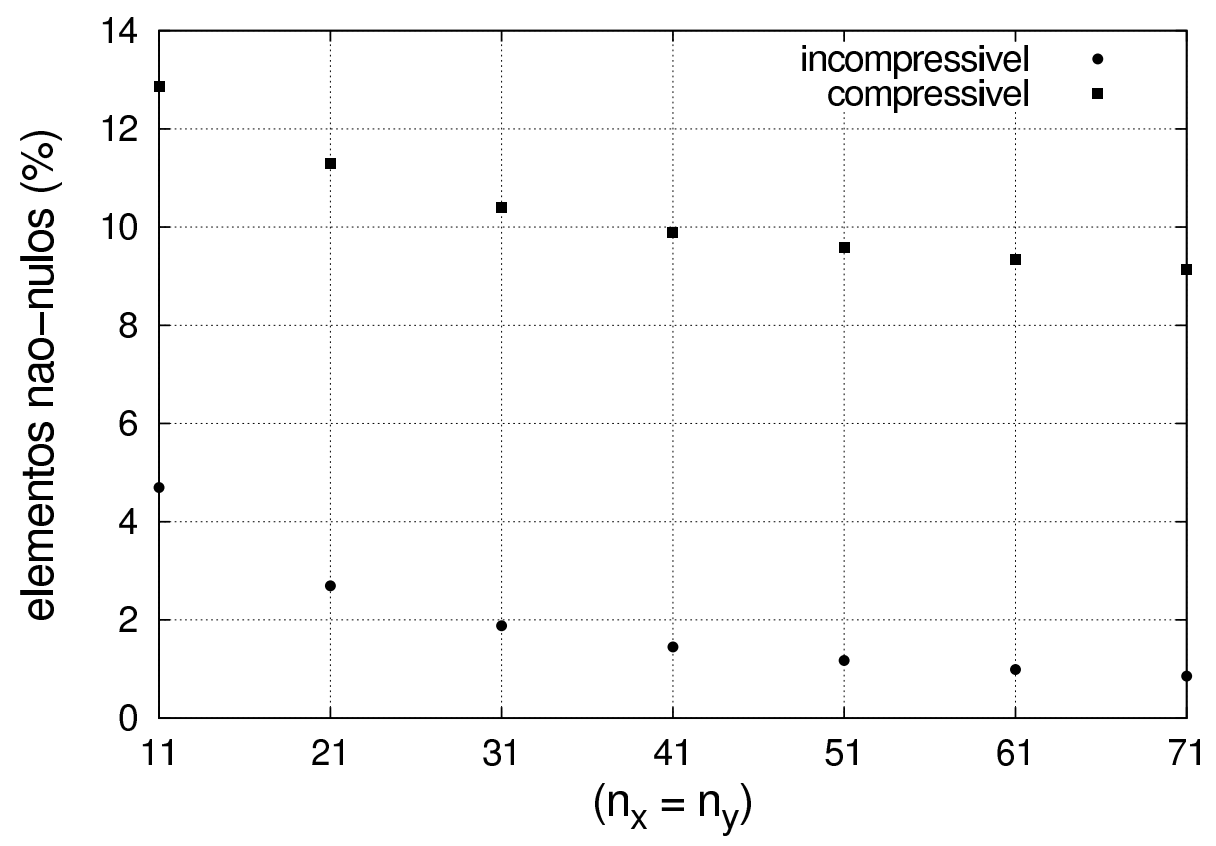

Figura 5.5: Porcetagem dos elementos não-nulos em relação aos elementos da matriz original dado por $\left(n_{x} \times n_{y} \times n_{\mathrm{v} a r}\right)^{2}$, onde $n_{\mathrm{v} a r}=4$ e $n_{\mathrm{v} a r}=5$ correspondem ao escoamento incompressível e compressível, respectivamente.

Pode ser visto na tabela 5.2 que independente do método utilizado, o número de elementos não-nulos da matriz original resultante da discretização do problema de estabilidade é maior para o caso compressível.

Uma comparação direta entre os casos compressíveis e incompressível pode ser visto na figura 5.5, onde ambas direções foram discretizadas por pontos de colocação espectral. Tomando-se uma matriz $n_{x}=n_{y}=61$, por exemplo, os elementos não-nulos são da ordem de $\mathrm{O}(1 \%)$ e $\mathrm{O}(9 \%)$, para os casos incompressível e compressível, respectivamente, uma diferença aproximada da ordem de $O(10)$. Entretanto, após o preenchimento da matriz e ordenação dos elementos, os elementos não-nulos tornaram-se da ordem de $\mathrm{O}(54 \%)$ e $\mathrm{O}(74 \%)$, como pode ser visto na tabela (5.2). Observou-se um incremento dos elementos não-nulos de quase 7 vezes para o caso compressível, embora o ganho relativo respectivo aos casos densos seja superior no caso incompressível. A discretização em pontos de Chebyshev-Gauss-Lobatto, permitiu representar as derivadas em alta precisão, entretanto o número dos coeficientes de preenchimento foi alto independentemente do método utilizado. 
No caso em que ambas direções são discretizadas por diferenças finitas, nota-se que mesmo com o preenchimento da matriz, o nível de esparsidade é grande em relação ao método espectral. Extraindo dados da tabela (5.3) para uma malha de $150 \times 150$ e considerando o melhor método de ordenação as relações foram as seguintes: 0.03\% de elementos não-nulos para $0.9 \%$ após a ordenação dos elementos para DF4 e, de $0.05 \%$ de elementos não-nulos para $1.7 \%$ após a ordenação para DF6. É notório a diferença discrepante entre os métodos de diferenças finitas e espectrais em relação ao nível de esparsidade da matriz mesmo após o preenchimento dos coeficientes. Entretanto, era esperado um resultado como este baseando-se nos dados apresentados na tabela (3.1).

Em certa medida, no que se refere à esparsidade da matriz, era esperado que a utilização de tipos diferentes de métodos de discretização para cada direção poderiam trazer resultados interessantes com valores intermediários aos apresentados anteriormente. De fato, fixando a ordem do método de diferenças finitas, as relações para ambos métodos apresentados, CGL-DFq e DFq-CGL, foram semelhantes, isto é, o aumento do número de elementos não-nulos após a ordenação e os coeficientes de preenchimento foi aproximadamente 8 e 10 vezes respectivamente aos métodos de ordem 4 e 6 , como pode ser visto em na figura (5.6). Além disso, o nível de esparsidade da matriz original era cerca de 1.2\% considerando um domínio computacional de $90 \times 90$.

Nota-se que, mesmo os casos em que o nível de esparsidade da matriz original era grande, após o preenchimento da matriz e ordenação o nível aumentou em até 30 vezes para os métodos DFq's. na figura (5.6).

Observa-se aqui uma importância muito grande da discretização espacial escolhida em relação ao grau de esparsidade da matriz, uma vez que o número de elementos não-nulos tem influência direta na performance da decomposição LU. Uma escolha criteriosa do método de discretização e do método de ordenação pode levar a ganhos computacionais significativos.

Ressalta-se que o operador linear (2.16) é muito particular, pois inclui derivadas de 

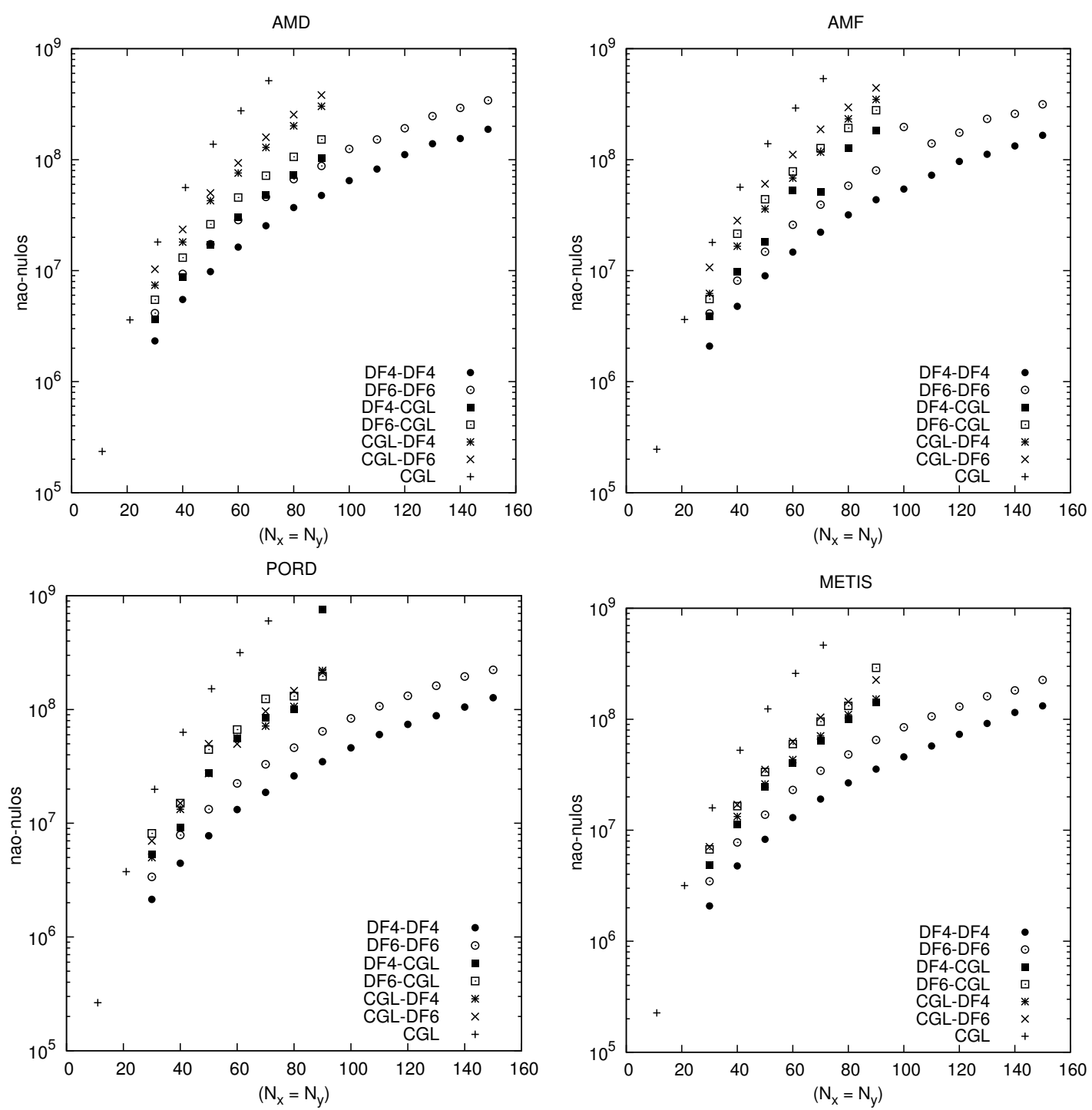

Figura 5.6: Coeficiente de preenchimento em função do método de ordenação e discretização em escala semi-logarítmica.

primeira e segunda ordem nas direções $x$ e $y$, além da derivada cruzada, $\frac{\partial^{2}}{\partial x y}$, presente somente no caso compressível, principal responsável pelo aumento dos coeficientes de preenchimento. Isso é devido à discretização espacial e ao produto de Kronecker que torna denso cada bloco da matriz. De forma ilustrativa, isto pode ser observado na figura (3.6-e) onde nota-se dois blocos em que não há elementos não-nulo considerando discretização em pontos de colocação, ou ainda, pelos dados apresentados na tabela (3.1).

Todos os resultados obtidos apresentados na seção 5.3 foram realizados utilizando METIS como método de reordenação da matriz. A partir das comparações entre os diferentes métodos de ordenação conclui-se que AMF e PORD não melhoraram o desempenho da 
solução para o problema físico de interesse no que diz respeito à solução densa e, portanto, foram descartados. O desempenho dos métodos AMD e METIS foram semelhantes, sendo este último um pouco superior para a maioria das combinações dos métodos de discretização aqui utilizados. Além disso, a existência de uma versão em paralelo das rotinas de ordenação METIS nos levou a escolha desse método.

A memória disponível em um determinado computador é geralmente o fator limitante na formação da matriz para soluções deste tipo de problemas de autovalor. A solução na versão densa de um problema de estabilidade linear compressível requer o armazenamento de uma matriz com dimensão $N=5 \times N_{x} \times N_{y}$, de modo que a quantidade de memória necessária escala da seguinte forma:

$$
\operatorname{Mem} \sim N^{2}
$$

independentemente do método de diferenciação.

A fim de mostrar os benefícios da utilização de técnicas de esparsas sobre a memória necessária, foi conveniente normalizar a memória a partir da quantidade de memória requerida para o armazenamento denso. Figura 5.7 apresenta a memória necessária escalonada para a fatoração numérica em função da dimensão para o caso denso equivalente para diferentes discretizações espaciais. Os dados referentes à memória computacional para os distintos meodos de discretização espacial utilizados neste trabalho podem ser vistos nas tabelas (5.5) e (5.6). A utilização de memória correspondente à abordagem densa $^{3}$ foi também mostrado na figura para efeito de comparação.

A combinação de uma discretização CGL-CGL com álgebra esparsa levou à uma modesta redução do fator de dois na memória necessária, independentemente da resolução. Por outro lado, quando, pelo menos, uma das direções espaciais foi discretizada pelo método das diferenças finitas, melhorias notáveis, em termos de eficiência, foram obtidos e a memória necessária para a fatorização escalonada exibe um decaimento quase constante

\footnotetext{
${ }^{3}$ Utilizou-se rotinas do pacote LAPACK para a decomposição LU da matriz densa.
} 

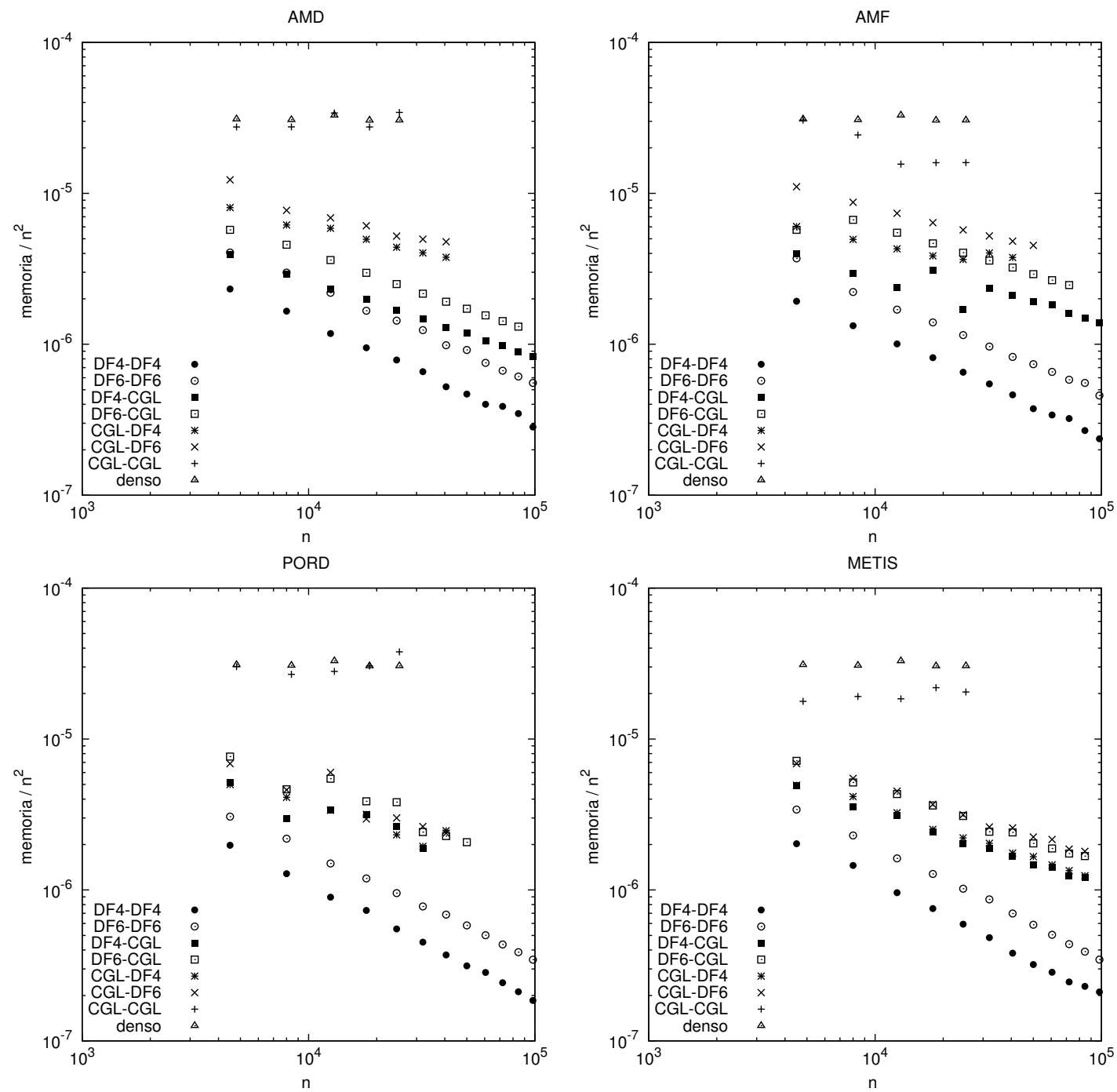

Figura 5.7: Estimativa da memória escalonada pela memória requerida no caso denso para os diferentes métodos de ordenação e discretização espacial.

com a dimensão da matriz:

$$
\operatorname{Mem} \sim N^{2}\left(k_{1}+10^{-k_{2} N}\right)
$$

onde $k_{1} \leq 1$ e $k_{2}$ são duas constantes positivas que dependem do método de diferenciação.

A derivação de uma expressão exata semelhante a (5.11) para a parte da formação da matriz do algoritmo e o passo da fatorização simbólica seria extremamente incômodo. Esta expressão aproximada, que, todavia, se encaixa razoavelmente bem aos resultados numéricos, pode ser útil para descrever a dependência da memória em relação ao método de diferenciação e estimar os recursos para cálculos futuros. 
Tabela 5.5: Step II: Fatoração simbólica. Estimativa da memória (Mb) requirida para a decomposição $L U$ para escoamento incompressível e diferentes métodos de ordenação.

\begin{tabular}{crrrrr}
\hline & \multicolumn{5}{c}{ Incompressível } \\
\cline { 2 - 6 } Grid & Denso & AMD & AMF & PORD & METIS \\
\hline $11 \times 11$ & 21 & 20 & 15 & 21 & 20 \\
$21 \times 21$ & 82 & 225 & 112 & 112 & 184 \\
$31 \times 31$ & 276 & 522 & 266 & 358 & 348 \\
$41 \times 41$ & 758 & 696 & 277 & 440 & 614 \\
$51 \times 51$ & 1741 & 1658 & 706 & 1013 & 1044 \\
$61 \times 61$ & 3491 & 2863 & 1218 & 1915 & 2088 \\
$71 \times 71$ & 5294 & 6093 & 2088 & 3266 & 4444 \\
\hline
\end{tabular}

Quando o método das diferenças finitas foi empregado em ambas as direções, a constante $k_{2}$ em (5.11) foi aproximadamente a mesma tanto para o DF4-DF4 como para DF6-DF6, o que significa que a economia de memória relativo ao incremento da resolução foram independentes da matriz utilizada. No entanto, o estêncil determina $k_{1}$ fazendo com que as DF's, de ordem inferior, fosse menos exigente para uma determinada resolução. Combinações entre os métodos de discretizações espacial por diferenças finitas e espectral apresentaram um comportamento semelhante às combinações DF-DF. Nota-se que a estrutura do operador discretizado usando pontos de colocação na direção $x$ e diferenças finitas na direção $y$ foi apenas um pouco diferente quando empregou-se diferenças finitas na direção $y$ e pontos de colocação na direção $x$.

Estas diferenças na estrutura da matriz afetam o desempenho do método de ordenação no preenchimento dos coeficientes, explicando as pequenas diferenças observadas na figura 5.7. A constante $k_{2}$ nestes casos foi novamente quase independente da matriz utilizada, mas a sua magnitude foi menor, ou seja, uma economia importante de memória importantes foi obtida com respeito a uma solução densa, porém menores que a economia obtida quandos usou-se esquemas de diferenças finitas em ambas direções (DF-DF).

A atenção está voltada agora para os tempos de CPU gastos na decomposição LU da matriz. Resultados para a fatoração esparsa utilizando-se o procedimento desenvolvido neste trabalho são indicados nas tabelas (5.7) e (5.8). 
Tabela 5.6: Step II: Fatoração simbólica. Estimativa da memória requirida para a decomposição $L U(\mathrm{Mb})$ para escoamento compressível e diferentes métodos de ordenação e discretização.

\begin{tabular}{crrrrr}
\hline & \multicolumn{5}{c}{ CGL-CGL } \\
\cline { 2 - 6 } Grid & Denso & AMD & AMF & PORD & METIS \\
\hline $11 \times 11$ & 11 & 11 & 11 & 11 & 11 \\
$21 \times 21$ & 153 & 133 & 706 & 123 & 102 \\
$31 \times 31$ & 716 & 635 & 358 & 696 & 410 \\
$41 \times 41$ & 2170 & 1945 & 1720 & 1894 & 1351 \\
$51 \times 51$ & 5571 & 5754 & 2641 & 4748 & 3123 \\
$61 \times 61$ & 10547 & 9524 & 5519 & 10547 & 7577 \\
$71 \times 71$ & 19384 & 21811 & 10147 & 23992 & 13025 \\
\hline
\end{tabular}

\begin{tabular}{crrrrrrrrr}
\hline & \multicolumn{4}{c}{ DF4-DF4 } & & \multicolumn{4}{c}{ DF6-DF6 } \\
\cline { 2 - 4 } \cline { 8 - 9 } Grid & AMD & AMF & PORD & METIS & & AMD & AMF & PORD & METIS \\
\hline $30 \times 30$ & 47 & 39 & 40 & 41 & & 82 & 75 & 62 & 69 \\
$40 \times 40$ & 106 & 85 & 82 & 93 & & 191 & 142 & 140 & 147 \\
$50 \times 50$ & 184 & 157 & 140 & 150 & & 343 & 265 & 234 & 253 \\
$60 \times 60$ & 307 & 264 & 237 & 244 & & 540 & 452 & 386 & 414 \\
$70 \times 70$ & 472 & 392 & 331 & 356 & & 860 & 690 & 572 & 612 \\
$80 \times 80$ & 674 & 559 & 462 & 495 & & 1271 & 989 & 796 & 887 \\
$90 \times 90$ & 857 & 759 & 609 & 625 & & 1616 & 1353 & 1127 & 1145 \\
$100 \times 100$ & 1170 & 935 & 786 & 801 & & 2292 & 1846 & 1455 & 1470 \\
$110 \times 110$ & 1466 & 1244 & 1040 & 1044 & & 2756 & 2401 & 1835 & 1848 \\
$120 \times 120$ & 2010 & 1669 & 1260 & 1274 & & 3463 & 3017 & 2257 & 2267 \\
$130 \times 130$ & 2482 & 1917 & 1511 & 1640 & & 4365 & 3961 & 2763 & 2789 \\
$140 \times 140$ & 2720 & 2273 & 1779 & 2027 & & 5325 & 4398 & 3314 & 3323 \\
$150 \times 150$ & 3319 & 2849 & 2173 & 2328 & & 6061 & 5241 & 3784 & 3912 \\
\hline
\end{tabular}

\begin{tabular}{|c|c|c|c|c|c|c|c|c|}
\hline \multirow[b]{2}{*}{ Grid } & \multicolumn{4}{|c|}{ DF4-CGL } & \multicolumn{4}{|c|}{ DF6-CGL } \\
\hline & AMD & $\mathrm{AMF}$ & PORD & METIS & AMD & $\mathrm{AMF}$ & PORD & METIS \\
\hline $20 \times 20$ & 28 & 28 & 27 & 27 & 35 & 35 & 41 & 41 \\
\hline $30 \times 30$ & 80 & 81 & 104 & 99 & 116 & 116 & 155 & 145 \\
\hline $40 \times 40$ & 187 & 189 & 189 & 227 & 292 & 427 & 297 & 330 \\
\hline $50 \times 50$ & 361 & 370 & 529 & 485 & 565 & 857 & 853 & 675 \\
\hline $60 \times 60$ & 640 & 1003 & 1025 & 787 & 963 & 1510 & 1253 & 1175 \\
\hline $70 \times 70$ & 1007 & 1025 & 1590 & 1221 & 1504 & 2429 & 2289 & 1856 \\
\hline $80 \times 80$ & 1507 & 2417 & 1931 & 1930 & 2218 & 3673 & 2477 & 2490 \\
\hline \multirow[t]{2}{*}{$90 \times 90$} & 2124 & 3469 & & 2743 & 3141 & 5284 & 3732 & 3945 \\
\hline & \multicolumn{4}{|c|}{ CGL-DF4 } & \multicolumn{4}{|c|}{ CGL-DF6 } \\
\hline Grid & AMD & $\mathrm{AMF}$ & PORD & METIS & AMD & $\mathrm{AMF}$ & PORD & METIS \\
\hline $30 \times 30$ & 163 & 122 & 101 & 100 & 249 & 224 & 139 & 139 \\
\hline $40 \times 40$ & 395 & 317 & 263 & 266 & 494 & 558 & 294 & 351 \\
\hline $50 \times 50$ & 916 & 670 & 527 & 507 & 1075 & 1152 & 938 & 705 \\
\hline $60 \times 60$ & 1607 & 1248 & 1025 & 816 & 1976 & 2070 & 957 & 1195 \\
\hline $70 \times 70$ & 2636 & 2188 & 1391 & 1329 & 3126 & 3432 & 1801 & 1891 \\
\hline $80 \times 80$ & 4131 & 4123 & 1992 & 2086 & 5089 & 5346 & 2695 & 2677 \\
\hline $90 \times 90$ & 6188 & 6158 & 4050 & 2877 & 7831 & 7906 & 3898 & 4229 \\
\hline
\end{tabular}


Tabela 5.7: Step III: CPU-time para fatorização (s) para o caso imcompressível.

\begin{tabular}{crrrrr}
\hline & \multicolumn{5}{c}{ CGL-CGL } \\
Grid CGL & Denso & AMD & AMF & PORD & METIS \\
\hline $11 \times 11$ & 0.1 & 0.0 & 0.0 & 0.0 & 0.0 \\
$21 \times 21$ & 2.3 & 0.8 & 1.0 & 0.7 & 1.0 \\
$31 \times 31$ & 28.4 & 5.5 & 6.7 & 5.6 & 6.9 \\
$41 \times 41$ & 152.7 & 28.5 & 30.7 & 27.1 & 34.7 \\
$51 \times 51$ & 553.9 & 99.8 & 104.0 & 96.0 & 97.0 \\
$61 \times 61$ & 1603.4 & 322.3 & 281.8 & 273.1 & 292.0 \\
$71 \times 71$ & 4665.8 & 866.7 & 660.3 & 666.0 & 1516.6 \\
\hline
\end{tabular}

Comparações também foram feitas aqui com o tempo equivalente necessário para se obter solução onde as matrizes foram tratadas como densa, para que uma escala de tempo que leva em conta a dimensão da matriz dada por:

$$
t \sim N^{3}
$$

onde $N$ é a dimensão da matriz.

Figura 5.8 mostra os tempos escalonados correspondentes às diferentes combinações de discretizações espaciais. Os valores para a solução densa também foram incluídos. À semelhança do que aconteceu para o uso da memória, a solução esparsa usando uma discretização CGL-CGL só proporcionou uma redução modesta no tempo de CPU, e sua eficiência não foi aumentada com incremento da resolução. Isto está de acordo com o preenchimento progressivo da matriz, ao abordar o problema de instabilidade do escoamento compressível, como pode ser visto nos padrões de esparsidade apresentados nas figuras (3.6 e 3.7) respectivas à discretização do operador linear em pontos de ChebyshevGauss-Lobatto. Por outro lado, melhorias notáveis foram obtidas quando empregou-se esquemas diferenças finitas em ambas direções (FD-FD) e uma combinação dos métodos diferenças finitas na direção $x$ e espectral na direção $y$ e suas permutações, de acordo com as melhorias na memória. Neste caso, o tempo da CPU pode ser aproximada como

$$
t \sim N^{3}\left(k_{3}+10^{-k_{4} N}\right)
$$

onde $k_{3} \leq 1$ e $k_{4}$ são novamente constantes positivas dependendo do método de discreti- 
Tabela 5.8: Step III:CPU-time para fatorização (s) para o caso compressível.

\begin{tabular}{crrrrr}
\hline & \multicolumn{5}{c}{ CGL-CGL } \\
\cline { 2 - 6 } Grid & Denso & AMD & AMF & PORD & METIS \\
\hline $11 \times 11$ & 0.1 & 0.1 & 0.1 & 0.1 & 0.1 \\
$21 \times 21$ & 2.3 & 1.8 & 2.1 & 1.7 & 1.2 \\
$31 \times 31$ & 23.6 & 16.9 & 23.9 & 21.3 & 13.1 \\
$41 \times 41$ & 124.1 & 99.6 & 119.5 & 119.4 & 75.6 \\
$51 \times 51$ & 456.9 & 349.3 & 426.5 & 428.1 & 262.1 \\
$61 \times 61$ & 1342.8 & 962.0 & 1228.8 & 1269.4 & 771.7 \\
$71 \times 71$ & - & - & 2924.4 & - & 2092.7 \\
\hline
\end{tabular}

\begin{tabular}{|c|c|c|c|c|c|c|c|c|}
\hline \multirow[b]{2}{*}{ Grid } & \multicolumn{4}{|c|}{ DF4-DF4 } & \multicolumn{4}{|c|}{ DF6-DF6 } \\
\hline & AMD & $\mathrm{AMF}$ & PORD & METIS & AMD & $\mathrm{AMF}$ & PORD & METIS \\
\hline $30 \times 30$ & 0.43 & 0.35 & 0.35 & 0.29 & 1.17 & 1.32 & 0.65 & 0.75 \\
\hline $40 \times 40$ & 1.30 & 1.09 & 0.82 & 0.89 & 3.38 & 2.68 & 2.04 & 2.03 \\
\hline $50 \times 50$ & 2.65 & 2.66 & 1.60 & 1.73 & 7.47 & 6.11 & 3.61 & 4.36 \\
\hline $60 \times 60$ & 5.30 & 5.32 & 3.47 & 3.06 & 14.07 & 13.06 & 8.51 & 8.86 \\
\hline $70 \times 70$ & 9.45 & 8.80 & 5.20 & 5.07 & 27.48 & 21.78 & 12.42 & 14.38 \\
\hline $80 \times 80$ & 15.54 & 13.56 & 7.85 & 7.38 & 44.35 & 40.83 & 18.45 & 22.30 \\
\hline $90 \times 90$ & 21.03 & 20.56 & 11.19 & 10.92 & 59.74 & 52.41 & 30.52 & 33.95 \\
\hline $100 \times 100$ & 32.85 & 25.17 & 16.90 & 14.54 & 106.73 & 92.17 & 48.87 & 43.50 \\
\hline $110 \times 110$ & 42.28 & 37.62 & 25.28 & 19.54 & 122.47 & 128.89 & 67.03 & 58.80 \\
\hline $120 \times 120$ & 68.07 & 57.78 & 31.03 & 27.95 & 168.56 & 169.78 & 74.40 & 77.68 \\
\hline $130 \times 130$ & 97.32 & 71.35 & 38.39 & 49.98 & 239.27 & 257.86 & 98.08 & 102.08 \\
\hline $140 \times 140$ & 99.78 & 79.30 & 48.21 & 58.76 & 304.30 & 267.52 & 122.79 & 126.57 \\
\hline $150 \times 150$ & 134.32 & 110.19 & 60.71 & 64.63 & 369.44 & 344.48 & 141.60 & 155.31 \\
\hline
\end{tabular}

\begin{tabular}{|c|c|c|c|c|c|c|c|c|}
\hline \multirow[b]{2}{*}{ Grid } & \multicolumn{4}{|c|}{ DF4-CGL } & \multicolumn{4}{|c|}{ DF6-CGL } \\
\hline & AMD & $\mathrm{AMF}$ & PORD & METIS & AMD & $\mathrm{AMF}$ & PORD & METIS \\
\hline $30 \times 30$ & 0.72 & 0.77 & 1.52 & 1.19 & 1.52 & 1.59 & 3.47 & 2.36 \\
\hline $40 \times 40$ & 2.26 & 2.60 & 2.81 & 3.47 & 4.98 & 14.05 & 6.35 & 7.32 \\
\hline $50 \times 50$ & 5.48 & 6.63 & 18.79 & 9.97 & 12.40 & 35.22 & 36.1 & 18.45 \\
\hline $60 \times 60$ & 12.14 & 35.75 & 37.16 & 19.17 & 26.43 & 74.69 & 62.35 & 40.53 \\
\hline $70 \times 70$ & 22.25 & 22.86 & 93.44 & 32.76 & 48.45 & 142.91 & 166.45 & 72.64 \\
\hline $80 \times 80$ & 37.87 & 108.40 & 71.96 & 60.90 & 85.00 & 248.70 & 118.16 & 106.71 \\
\hline $90 \times 90$ & 60.01 & 171.61 & & 98.08 & 129.30 & 427.96 & 205.13 & 203.61 \\
\hline \multirow[b]{2}{*}{ Grid } & \multicolumn{4}{|c|}{ CGL-DF4 } & \multicolumn{4}{|c|}{ CGL-DF6 } \\
\hline & AMD & AMF & PORD & METIS & AMD & $\mathrm{AMF}$ & PORD & METIS \\
\hline $30 \times 30$ & 2.92 & 2.54 & 1.30 & 1.21 & 5.74 & 7.30 & 2.42 & 2.59 \\
\hline $40 \times 40$ & 9.47 & 9.58 & 4.90 & 4.83 & 15.11 & 25.42 & 6.23 & 8.17 \\
\hline $50 \times 50$ & 32.63 & 27.09 & 13.42 & 11.22 & 45.30 & 68.28 & 40.20 & 17.17 \\
\hline $60 \times 60$ & 73.09 & 62.85 & 33.97 & 20.66 & 105.74 & 156.26 & 26.86 & 44.11 \\
\hline $70 \times 70$ & 141.93 & 152.89 & 41.78 & 40.23 & 212.20 & 307.58 & 76.53 & 85.99 \\
\hline $80 \times 80$ & 274.15 & 373.11 & 70.00 & 72.18 & 422.95 & 580.01 & 129.66 & 126.00 \\
\hline $90 \times 90$ & 500.58 & 657.93 & 228.07 & 111.32 & 753.72 & 998.37 & 220.9 & 242.97 \\
\hline
\end{tabular}


zação espacial.
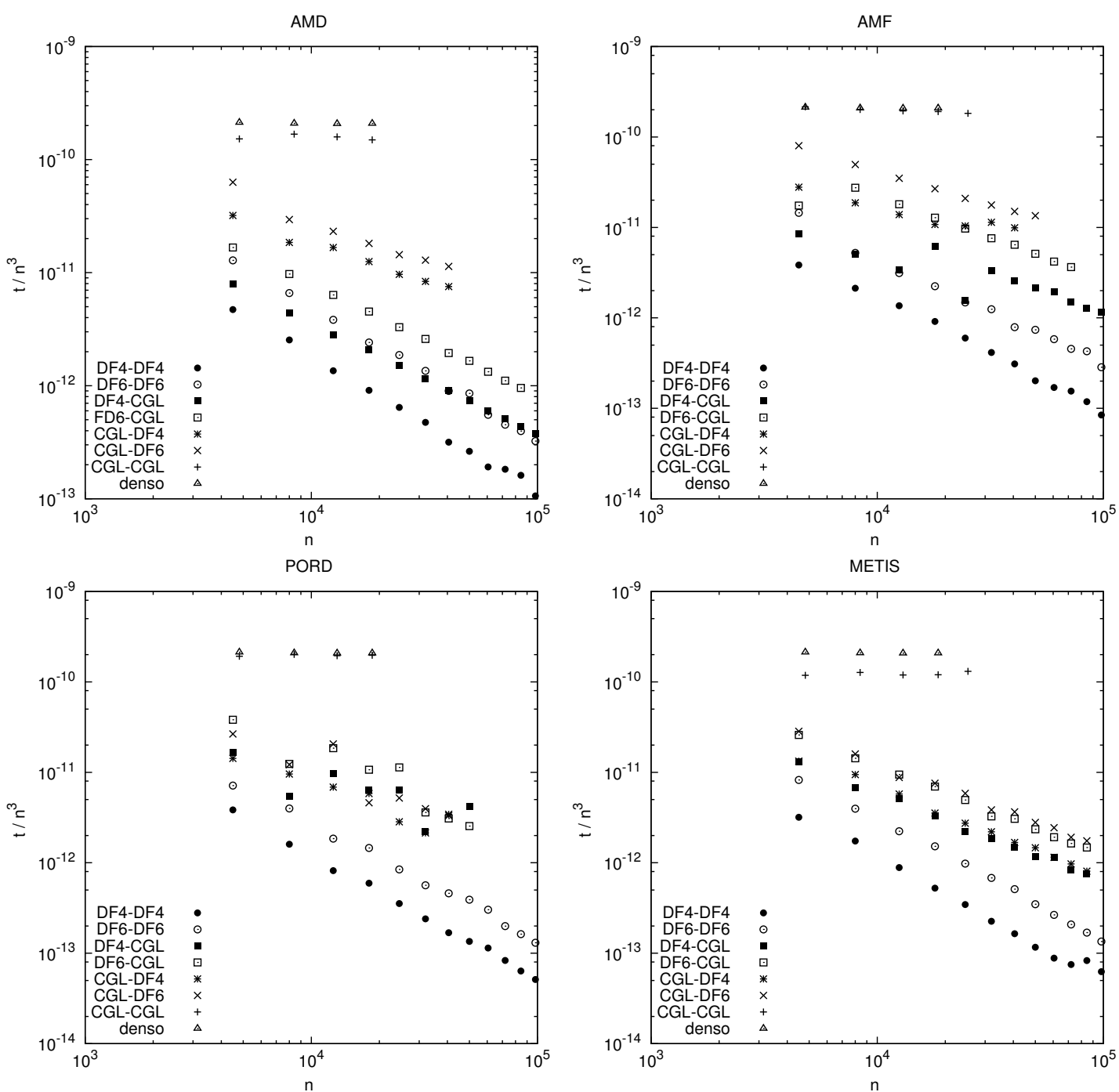

Figura 5.8: Tempo de processamento (CPU) escalado com a memória requerida na solução densa $\left(N^{3}\right)$, para diferentes resoluções, métodos e discretizações.

As melhorias de desempenho, tanto em tempo e como em memória, obtidas com o uso de álgebra esparsa foram notáveis quando esquemas de diferenças finitas foram utilizados para a discretização espacial para um determinado número de pontos de diferenciação. No entanto, a maior exatidão dos métodos de diferenciação utilizado menor é o número de pontos necessários para a convergência dos resultados físicos. Este compromisso implica que, a fim de determinar a melhor prática para a solução esparsa do problema de autovalor, a capacidade de resolução da discretização espacial deve ser levada em conta em conjunto com a eficiência da solução esparsa. Isso requer um caso de teste para um escoamento 
adequado.

O problema da instabilidade de uma camada limite ao longo de uma linha de estagnação de uma asa incompressível estão presentes na literatura foi tomado como um problema teste. Nota-se que a forma particular do escoamento base e das perturbações para este problema físico, em que as variações ao longo da direção y são muito mais fortes do que aquelas ao longo da direção $x$, facilitaria a solução numérica se os dois sentidos fossem tratados de maneira diferente.

Esta particularidade deste problema não foi explorado aqui, a fim de obter resultados de desempenho aplicáveis a qualquer outro problema físico. Resoluções de até 60 pontos de colocação espectrais por direção espacial (CGL-CGL) foram necessárias para a convergência de 8 casas decimais do autovalor dominante, para alguma combinação arbitrária de parâmetros físicos. O erro relativo correspondente aos diferentes métodos de discretizações em relação ao valor de referência são mostrados na Figura (5.9).

Quando o método de discretização utilizado foi o mesmo em ambas direções (CGLCGL, DF4-DF4 e DF6-DF6), distintas taxas de convergência para cada método de diferenciação de ordem superior foram obtidas. O interesse aqui foi determinar a resolução necessária para cada um dos métodos, a fim de convergir os autovalores para o mesmo erro relativo. Estas resoluções são mostrados na tabela (5.9), juntamente com a memória e tempo de CPU associado ao método de solução esparsa. Os valores que correspondem a uma solução utilizando discretização CGL-CGL em ambas direções e abordagem densa também são mostrados.

Como esperado, a resolução necessária para a convergência do autovalor para a discretização DF4-DF4 foi muito mais elevada do que a correspondente à discretização CGLCGL, e seria suficiente para descartar o método de solução por álgebra denso. No entanto, quando se olha para os requisitos de memória, os dois casos extremos, CGL-CGL e DF4DF4, apresentaram valores semelhantes, enquanto o tempo associado com DF4-DF4 foi menos da metade do que o tempo empregado para a solução usando discretização CGLCGL, e menos de um quarto do que a solução mais eficaz para álgebra densa. O melhor 


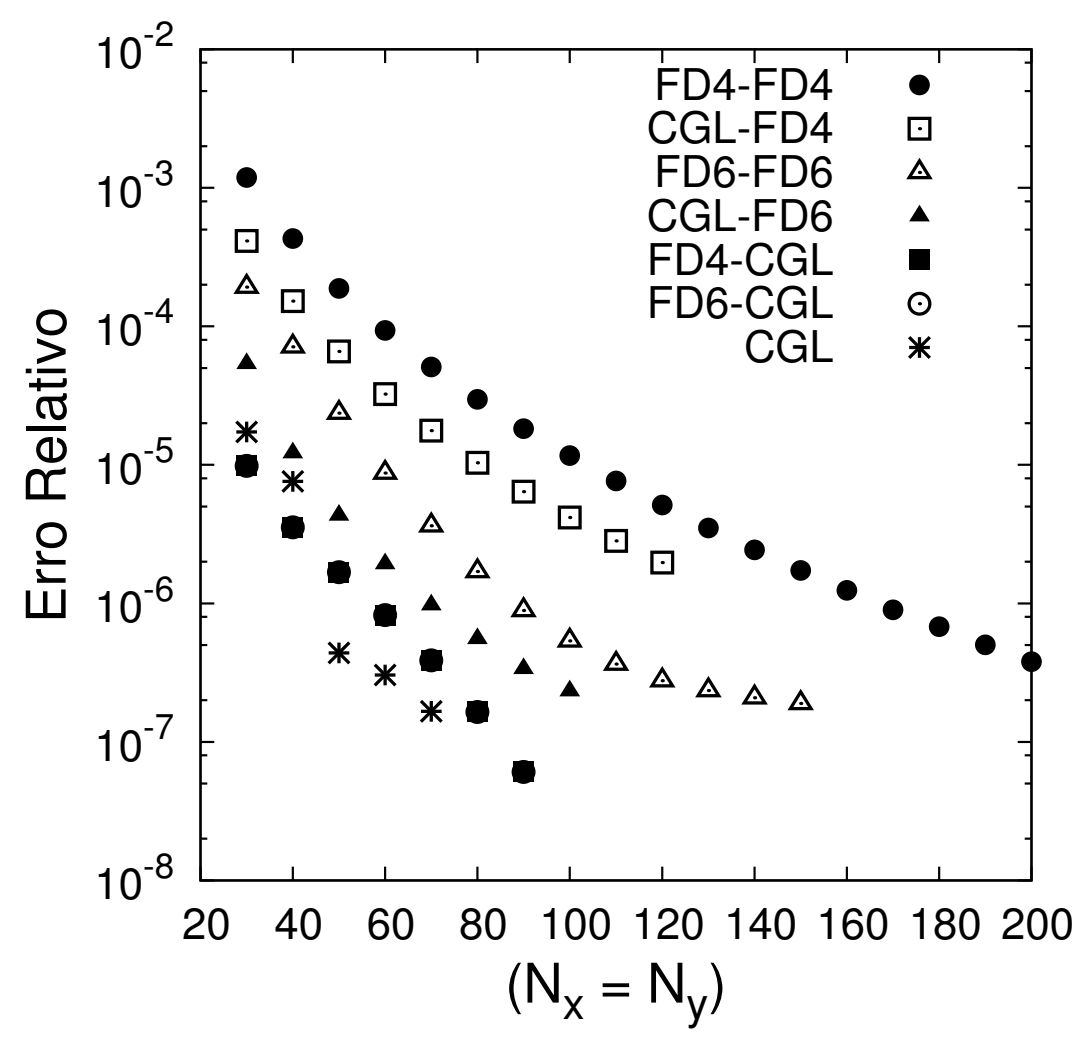

Figura 5.9: Distribuição do erro relativo referente ao modo GH para $M a=0.9, R e=800$ e $\beta=0.19$ correspondente a cada distretização espacial.

desempenho, no entanto, foi alcançado para a discretização DF6-DF6 que acabou por ser a melhor solução de compromisso, com um tempo de CPU de uma ordem de grandeza menor do que as outras alternativas.

Conclui-se que, ao abordar um problema físico no qual as duas resoluções espaciais têm comportamento semelhante, a melhor prática (entre as possibilidades estudadas aqui) para uma metodologia que baseia-se em formar a matriz esparsa é o uso de diferenças finitas de $6^{a}$ ordem. Resultados usando DF8, não mostrado aqui, são marginalmente melhor em precisão, tornando o desempenho claramente mais pobre.

Em muitos problemas os gradientes das grandezas físicas ao longo de uma direção são muito mais fortes do que ao longo do outro. Nestes casos, as melhorias importantes na solução numérica pode ser obtida escolhendo diferentes discretizações para os dois sentidos. Para este problema do escoamento ao longo do bordo de ataque de uma asa 
enflechada, uma resolução muito fina é necessária para resolver a camada limite, mas as variações do escoamento base e perturbação ao longo da direção $x$ são suaves. Os resultados apresentados na figura (5.9) e tabela (5.9) mostram as combinações possíveis usando o mesmo número de pontos de discretização ao longo de ambas as direções. Notase que o uso de uma discretização espectral ao longo da direção mais exigente, direção y, e uma discretização por diferenças finitas ao longo da direção mais fácil de resolver ofereceu um desempenho ainda melhor. Além disso, se o número de pontos da discretização fosse variado de forma independente para os dois sentidos, outras melhorias no desempenho numérico poderia ser alcançado. Estes refinamentos, no entanto, são dependentes do problema e uma regra geral não é possível.

Tabela 5.9: Memória e tempo de CPU para a solução do problema de autovalor BiGlobal para a convergência do modo dominante acima de 6 casas decimais, para uma combinação de métodos de discretização. O problema físico testado é o escoamento ao longo do bordo de ataque de uma asa enflechada em $R e=800, M a=0.9$ e $\beta=0.19$.

\begin{tabular}{cccc}
\hline Método & Resolução & Memória $(\mathrm{Mb})$ & tempo CPU (s) \\
\hline CGL - CGL (denso) & $50 \times 50$ & 5177 & 456.9 \\
\hline CGL - CGL & $50 \times 50$ & 3123 & 262.1 \\
DF6 - DF6 & $90 \times 90$ & 1145 & 33.95 \\
DF4 - DF4 & $170 \times 170$ & 3238 & 111.28 \\
\hline CGL - DF4 & $120 \times 120$ & 6960 & 362.21 \\
CGL - DF6 & $70 \times 70$ & 1891 & 220.90 \\
DF4 - CGL & $60 \times 60$ & 640 & 12.14 \\
DF6 - CGL & $50 \times 50$ & 565 & 12.4 \\
\hline
\end{tabular}

\subsection{Resultados da análise de estabilidade BiGlobal}

Nesta seção são apresentados resultados da análise da instabilidade linear para o escoamento compressível de escoamento sobre um bordo de ataque de uma asa enflechada.

Inicialmente, verificou-se e realizou-se testes do procedimento desenvolvido neste trabalho com vistas aos resultados de referência que a literatura apresenta.

Para o caso incompressível, o modo Görtler-Hämmerlin (GH) das perturbações lineares foi primeiramente adotado por Hall, Malik e Poll (1984), e recuperado mais tarde, 
Tabela 5.10: Histórico do refinamento da malha computacional da solução numérica da camada limite de uma linha de estagnação incompressível $(M a=0.02)$ de um bordo de ataque de uma asa em $R e=800, \beta=0.255$ e $x \in[-100,+100]$.

\begin{tabular}{c|cc|cc}
\hline & \multicolumn{2}{|c|}{ modo Görtler-Hämmerlin } & \multicolumn{2}{c}{ modo A1 } \\
Resolução & $c_{r}$ & $c_{i}$ & $c_{r}$ & $c_{i}$ \\
\hline $08 \times 48$ & 0.3584414 & 0.0058564 & 0.3573547 & 0.0043549 \\
$16 \times 48$ & 0.3584414 & 0.0058640 & 0.3580007 & 0.0042997 \\
$24 \times 48$ & 0.3584414 & 0.0058564 & 0.3458880 & 0.0043912 \\
$32 \times 48$ & 0.3584414 & 0.0058564 & 0.3580521 & 0.0041461 \\
$48 \times 48$ & 0.3584414 & 0.0058564 & 0.3579257 & 0.0040956 \\
$60 \times 48$ & 0.3584415 & 0.0058565 & 0.3579401 & 0.0040412 \\
$60 \times 60$ & 0.3584414 & 0.0058555 & 0.3579401 & 0.0040403 \\
$($ LIN; MALIK, 1996) & 0.3584098 & 0.0058325 & 0.3579170 & 0.0040988 \\
\hline
\end{tabular}

no contexto de análise de instabilidade global, por Lin e Malik (1996) e Theofilis et al. (2003). Com objetivo de reproduzir estes resultados com o presente código (compressível), um número de Mach muito baixo, $M a=0.02$, foi considerado.

A tabela (5.10) apresenta um histórico de convergência do modo mais instável (GH) e do primeiro modo antisimétrico (A1) obtidos neste trabalho. Estes resultados são comparados com valores de referência apresentados na literatura (LIN; MALIK, 1996). Note que os autovalores $c=\omega / \beta$ são apresentados na tabela, ao invés de $\omega$. Além do número de Mach, os parâmetros considerados são: número de Reynolds e número de onda na direção $z$, respectivamente, $R e=800$ e $\beta=0.255$. Nota-se uma diferença entre os valores obtidos em relação aos resultados apresentados por Lin e Malik (1996) e a razão disso pode ser atribuída ao valor finito para o número de Mach $(M a=0.02)$.

Outro aspecto investigado aqui foi a forma da autofunção. Para tanto, em primeiro lugar o efeito do tamanho do domínio e as condições de contorno foram abordados. Note que os resultados já estão convergidos em uma malha grosseira, tanto para os modos GH e A1 (primeiro modo antissimétrico). Além disso, observa-se que as condições de contorno adotadas, especialmente extrapolação linear, descritas e discutidas na seção 5.2 foram adequadas mesmo para as autofunções que não apresentam um comportamento linear direção-x. Resultados de um teste adicional são mostrados nas tabelas (5.11) e 
(5.12), onde a convergência dos três principais autovalores foi estudado com o aumento da extensão de domínio na direção $x$ em $M a=0,9$ e refinamento de malha. Um domínio relativamente grande $L_{x}=100$ foi necessário para a convergência, até mesmo em uma malha grosseira, para uma convergência do terceiro modo (S2) até a sexta casa decimal.

Os componentes de velocidades na direção $x$ e $z$ das autofunções correspondentes aos três principais modos são mostradas na figura (5.10) para $M a=0.9$. Trabalhos anteriores, porém para escoamento incompressível (LIN; MALIK, 1996; THEOFILIS et al., 2003), mostram que os principais modos são caracterizados por sua dependência polinomial na direção $x$ : o $k$-ésimo modo apresenta uma velocidade $\hat{u} \sim x^{k}$, enquanto as outras componentes de velocidade, pressão e temperatura são descritas por $\hat{v}, \hat{w}, \hat{p}, \hat{\theta} \sim x^{k-1}$. As autofunções mostrado na figura 5.10 confirmaram que o comportamento conhecido para o caso em que o escoamento em regime incompressível persiste também no regime compressível subsônico. A dependência particularmente simples das autofunções, respectivas aos primeiros modos na direção $x$, explica o bom comportamento das condições de contorno utilizadas quando o domínio computacional é bastante grande e a possibilidade de convergência de autovalores com resoluções relativamente baixas nesta direção. Isto explica também porque os autovalores recuperados não experimentaram mudanças substanciais quando o comprimento do domínio $x$ foi aumentado, em contraste com análise de estabilidade BiGlobal de escoamentos sem contornos em geral.

Tabela 5.11: Estudo de independência do domínio computacional em $R e=800, M a=0.90$ e $\beta=0.19$ para uma malha computacional $140 \times 140$ (DF6-CGL). Domínio abaixo de $[-100,+100]$ fornece resultados pobres para modo S2.

\begin{tabular}{ccccccc}
\hline & \multicolumn{2}{c}{ modo GH } & \multicolumn{2}{c}{ modo A1 } & \multicolumn{2}{c}{ modo S2 } \\
\cline { 2 - 7 }$x$-Domínio & $\omega_{r}$ & $\omega_{i}$ & $\omega_{r}$ & $\omega_{i}$ & $\omega_{r}$ & $\omega_{i}$ \\
\hline$[-25,+25]$ & 0.07647187 & 0.00118939 & 0.07636698 & 0.00057490 & - & - \\
{$[-50,+50]$} & 0.07647183 & 0.00118941 & 0.07634513 & 0.00057441 & - & - \\
{$[-75,+75]$} & 0.07647184 & 0.00118941 & 0.07633181 & 0.00057393 & - & - \\
{$[-100,+100]$} & 0.07647184 & 0.00118942 & 0.07632667 & 0.00057823 & 0.07618317 & -0.00003718 \\
{$[-125,+125]$} & 0.07647184 & 0.00118942 & 0.07633045 & 0.00057620 & 0.07618652 & -0.00003770 \\
{$[-150,+150]$} & 0.07647184 & 0.00118942 & 0.07632651 & 0.00058743 & 0.07618416 & -0.00004249 \\
\hline
\end{tabular}

A estabilidade linear do compressível de uma camada limite ao longo da linha de estag- 
Tabela 5.12: Estudo de refinamento de malha computacional na solução numérica para a camada limite de uma linha de estagnação compressível no domínio [-100,100] para $R e=800, M a=0.90$ e $\beta=0.19$ para o cálculo dos 3 primeiros modos GH, A1 e S2.

\begin{tabular}{ccccccc}
\hline & \multicolumn{2}{c}{ modo GH } & \multicolumn{2}{c}{ modo A1 } & \multicolumn{2}{c}{ modo S2 } \\
\cline { 2 - 7 } Resolução & $\omega_{r}$ & $\omega_{i}$ & $\omega_{r}$ & $\omega_{i}$ & $\omega_{r}$ & $\omega_{i}$ \\
\hline $40 \times 40$ & 0.07647188 & 0.00118982 & 0.07632863 & 0.00057917 & 0.07537234 & -0.00003475 \\
$50 \times 50$ & 0.07647180 & 0.00118961 & 0.07632886 & 0.00057692 & 0.07618187 & -0.00002476 \\
$60 \times 60$ & 0.07647180 & 0.00118951 & 0.07632883 & 0.00057664 & 0.07618766 & -0.00003697 \\
$70 \times 70$ & 0.07647181 & 0.00118947 & 0.07632884 & 0.00057658 & 0.07618845 & -0.00003738 \\
$80 \times 80$ & 0.07647182 & 0.00118944 & 0.07632885 & 0.00057656 & 0.07618847 & -0.00003724 \\
$90 \times 90$ & 0.07647183 & 0.00118943 & 0.07632885 & 0.00057655 & 0.07618845 & -0.00003716 \\
$100 \times 100$ & 0.07647184 & 0.00118942 & 0.07633004 & 0.00057603 & 0.07618600 & -0.00003618 \\
$110 \times 110$ & 0.07647184 & 0.00118942 & 0.07632887 & 0.00057654 & 0.07618835 & -0.00003690 \\
$120 \times 120$ & 0.07647184 & 0.00118942 & 0.07632910 & 0.00057660 & 0.07618877 & -0.00003745 \\
$130 \times 130$ & 0.07647184 & 0.00118941 & 0.07632896 & 0.00057489 & 0.07618604 & -0.00003726 \\
$140 \times 140$ & 0.07647184 & 0.00118942 & 0.07632667 & 0.00057823 & 0.07618316 & -0.00003718 \\
\hline
\end{tabular}

nação do bordo de ataque de uma asa, será apresentada agora, revisitando os resultados apresentados em (THEOFILIS; FEDOROV; COLLIS, 2004). O trabalho referenciado apresenta uma análise de estabilidade BiGlobal usando álgebra linear densa e compara seus resultados para os modos GH com os resultados obtidos a patir de uma expansão assintótica das equações de estabilidade local para dois números de Mach $(M a=0,5$ e $0,9)$, dois números de Reynolds $(R e=800$ e $R e=1500)$, e uma faixa de números de onda na direção $z \beta$. Seus cálculos demonstram um desacordo consistente entre os resultados fornecidos pelas duas abordagens, sendo que a causa mais provável foi a resolução limitada devido a abordagem numérica e computacional utilizada. A mesma combinação de parâmetros foi considerado aqui para verificar essa possibilidade. A taxa de amplificação calculada usando a presente abordagem são representados na figura (5.11) sobreposta aos resultados apresentados por Theofilis, Fedorov e Collis (2004). Observa-se uma perfeita concordância entre as previsões das análises assintóticas, denotadas por linhas na figura, e as análises de estabilidade BiGlobal foi alcançada, o que indica que as soluções numéricas encontradas por (THEOFILIS; FEDOROV; COLLIS, 2004) estão realmente sub-resolvidas. A concordância obtida aqui com os resultados "analíticos" acentua ainda mais a qualidade da presente implementação em termos de precisão numérica e eficiência 
computacional.

Figura 5.12 apresenta as taxas de amplificação temporal obtidas em três diferentes números de Reynolds $(R e=585,800$ e 1500) como função de $\beta$, para uma faixa de números de Mach, $M a \in[0.02,0.9]$. Para números de Reynolds muito instáveis (figuras 5.12a e 5.12b) a compressibilidade estabiliza os autovalores mais instáveis e torna mais estreita a faixa de número de ondas $\beta$ dominante, de modo que ele se torna menos amplificado e com comprimento característico menor na direção $z$. Essa estabilização foi mais pronunciada com número de Reynolds maiores. Este é o comportamento usual de estabilidade linear associado a compressibilidade, camada limite por exemplo. Entretanto, nas imediações dos valores críticos de $R e$, o efeito de compressibilidade foi invertido. Figura (5.12c), mostra que para $R e=585$ o incremento de $M a$ desestabilizou o modo que tem maior taxa de amplificação temporal. Uma discussão dos efeitos de compressibilidade nas condições críticas é apresentado mais adiante.

As figuras 5.13(a) e 5.13(b) mostram as taxas de amplificação/decaimento $\left(\omega_{i}\right)$ para uma frequência na qual foi encontrado um crescimento linear para todos os três modos mais amplificados dentro da faixa de número de Reynolds examinada. Note que a velocidade de fase é a inclinação da linha nos gráficos 5.13(c) e 5.13(d) e que portanto não variam com números de Reynolds e Mach. Estes resultados podem ser usados na identificação de autovalores em trabalhos experimentais bem como em simulações numéricas diretas. Realizando um pós-processamento elementar dos resultados obtidos temos a seguinte dependência linear entre as frequências do modos mais instável (ou menos estável) e o comprimento de onda transversal, $L_{z}=2 \pi / \beta$,

$$
\omega_{r}=\kappa L_{z}+\lambda
$$

onde $\kappa$ e $\lambda$ são parâmetros apresentados nas tabelas (5.13) e (5.14). A velocidade de fase é dada por $\kappa$.

A metodologia apresentada neste trabalho também pode ser aplicada ao regime transónico. Os resultados encontrados para um pequeno conjunto de parâmetros foram com- 
Tabela 5.13: Dependência de $\kappa$ em $M a$ e $R \boldsymbol{e}$.

\begin{tabular}{cccccc}
\hline & \multicolumn{2}{c}{$M a=0.5$} & & \multicolumn{2}{c}{$M a=0.9$} \\
\cline { 2 - 3 } \cline { 5 - 6 } Mode & $R e=700$ & $R e=800$ & & $R e=700$ & $R e=800$ \\
\hline GH & $4.52 \mathrm{E}-01$ & $4.42 \mathrm{E}-01$ & & $4.86 \mathrm{E}-01$ & $4.78 \mathrm{E}-01$ \\
A1 & $4.52 \mathrm{E}-01$ & $4.42 \mathrm{E}-01$ & & $4.86 \mathrm{E}-01$ & $4.78 \mathrm{E}-01$ \\
S2 & $4.52 \mathrm{E}-01$ & $4.39 \mathrm{E}-01$ & & $4.85 \mathrm{E}-01$ & $4.77 \mathrm{E}-01$ \\
\hline
\end{tabular}

Tabela 5.14: Dependência de $\lambda$ em $M a$ e $R \boldsymbol{e}$.

\begin{tabular}{cccccc}
\hline & \multicolumn{2}{c}{$M a=0.5$} & & \multicolumn{2}{c}{$M a=0.9$} \\
\cline { 2 - 3 } \cline { 5 - 6 } Mode & $R e=700$ & $R e=800$ & & $R e=700$ & $R e=800$ \\
\hline GH & $-1.70 \mathrm{E}-02$ & $-1.62 \mathrm{E}-02$ & & $-1.46 \mathrm{E}-02$ & $-1.42 \mathrm{E}-02$ \\
A1 & $-1.71 \mathrm{E}-02$ & $-1.62 \mathrm{E}-02$ & & $-1.47 \mathrm{E}-02$ & $-1.43 \mathrm{E}-02$ \\
S2 & $-1.73 \mathrm{E}-02$ & $-1.56 \mathrm{E}-02$ & & $-1.48 \mathrm{E}-02$ & $-1.43 \mathrm{E}-02$ \\
\hline
\end{tabular}

pilados na figura 5.14. Os resultados foram verificados a partir de Alexander Fedorov que usou a teoria assintótica, a mesma apresentada em (THEOFILIS; FEDOROV; COLLIS, 2004). Os casos selecionados que tiveram excelente concordância tinha como hipótese $\beta R e \gg 1$. Estudos mais aprofundados poderiam ser realizados para escoamentos em regimes transónicos com velocidade superior a velocidade do som.

A figura (5.15) apresenta taxas de amplificação para um número de onda e número de Reynolds fixos em 2 números de Mach. Observa-se que o comportamento apresentado não confirma a conjectura apresenta por Duc, Sesterhenn e Friedrich (2006) que versa uma maneira de escalar os valores da taxa de amplificação com $M a^{2}$. Provavelmente, o número pequeno de simulações apresentados por Duc, Sesterhenn e Friedrich (2006) talvez seria insuficiente para formular tal conjectura.

A influência da compressibilidade nos modos de instabilidade é estudado a seguir. Nota-se que na literatura que aborda a instabilidade linear global de escoamentos compressíveis para a camada limite ao longo de uma linha de estagnação de uma asa não faz um estudo sistemático, tampouco um estudo paramétrico completo. O estudo aqui foi restringido para escoamento subsónico, com número de Mach em escala $M a \in[0.02,0.9]$, e número de onda na direção $z$ até $\beta=0.3$, o que corresponde a um comprimento de peri- 
odicidade $L_{z} \approx 21$. Na faixa dos parâmetros analisados o modo GH foi o modo dominante encontrado.

Visto de uma outra forma, a figura 5.16 mostra a taxa de crescimento correspondente ao modo mais amplificado em cada combinação de Ma e Re, além de todos os números de ondas $\beta$. O foco no estudo nos autovalores e negligenciando os outros números de onda é justificado na estabilidade modal linear por causa do crescimento exponencial temporal. A taxa de crescimento aumenta monotonicamente com o número de Reynolds para todos $M a$, como esperado para uma instabilidade de origem viscosa. O número de Mach tem um efeito estabilizador quando $R e>700$, de forma consistente com os resultados da literatura (MACK, 1984) que versa sobre camada limite compressível. No entanto, este comportamento foi invertido em torno $R e=650$ e, para os números de Reynolds inferiores, a compressibilidade é desestabilizador, um resultado que difere dos resultados conhecidos para a camada limite sobre uma placa plana.

A figura (5.17) apresenta curvas de distribuição de $\beta$ versus número de Reynolds para cada número de Mach calculado. O comportamento apresentado das curvas sugere que deve existir um fator de escala em que houvesse um colapsamento das curvas. Entretanto, esse fator de escala não foi encontrado. Os resultados foram obtidos na máxima taxa de amplificação temporal. Uma decrescimento monotônico de $\beta$ (implicando um aumento no comprimento periodicidade spanwise) foi alcançada mediante o aumento Reynolds e números de Mach.

O efeito do incremento do número Mach nas autofunções foi mostrado na Figura 5.18, entretanto, somente a velocidade $w$ e a temperatura são mostrados. Como foi mostrado na figura 5.10, essas componentes são constantes ao longo da direção $x$ de maneira que para um corte na direção $y$, i.e, normal à parede, contém a informação essencial. Para efeitos de comparação, as autofunções foram normalizadas pelo pico de velocidade $w$. Sob esta normalização, a componente de velocidade foi mostrado ser independente em relação ao número de Mach na vizinhança da parede, até a região onde ocorre a mudança de fase. No pico da região externa, a magnitude aumenta ligeiramente com Ma. Além disso, 
as amplitudes relativas à temperatura tiveram notavelmente aumentos com o incremento de $M a$, como foi também o caso para o escoamento base. Isto sugere que as tendências observadas no comportamento de estabilidade são devido principalmente às alterações da viscosidade, que são por sua vez, devido ao aumento de temperatura associado a compressibilidade.

Figura (5.19) apresenta as curvas curvas neutras de estabilidade deste escoamento para a faixa de número de Mach considerada. As curvas apresentadadas na figura (5.19) são plotes de segmentos de pontos sem qualquer interpolação ou suavização das curva. A figura também apresenta resultados referentes a estabilidade neutra de trabalhos anteriores, destacados na figura pelos símbolos. Nota-se claramente um grande avanço com esse estudo paramétrico mais completo e sistemático.

De acordo com os resultados para o caso incompressível, o escoamento de uma camada limite ao longo da linha de estagnação do bordo de ataque de uma asa é apenas instável em relação à perturbações com comprimento finito e periodicidade na direção $z$. Como era esperado na Figura 5.17, o número de Reynolds crítico $\left(R e_{c r i t}\right)$ diminui monotonicamente com o aumento do número de Mach, pelo menos no intervalo de números de Mach subsónico considerado: o número de Reynolds é crítico reduzido de $R e_{\text {crit }} \approx 583$ a $M a=0,02$ para $R e_{c r i t} \approx 565$ à $M a=0,9$. Esta diminuição do número de Reynolds crítico é acompanhada por uma redução no número de onda crítico na direção $z \beta_{c r i t}$. O número de Reynolds e número de ondas em condições críticas para cada número de Mach são mostrados na Tabela 5.15. O comportamento desses valores também podem ser vistos nas figuras (5.20) e (5.21). Um resultado significativo é que o incremento do número de Mach reduz o número de Reynolds crítico de $R e_{\text {crit }} \approx 583 \mathrm{em} M a=0.02$ para $R e_{\text {crit }} \approx 565$ em $M a=0.9$. 
Tabela 5.15: Valores críticos $\left(R e_{c r i t}\right.$ e $\left.\beta_{\text {crit }}\right)$ correspondente vários números de Mach.

\begin{tabular}{c|c|c}
\hline Ma & Re $_{\text {crit }}$ & $\beta_{\text {crit }}$ \\
\hline $\mathbf{0 . 0 2}$ & $\mathbf{5 8 3 . 1}$ & $\mathbf{0 . 2 8 7 8}$ \\
0.1 & 582.7 & 0.2868 \\
0.2 & 581.6 & 0.2836 \\
0.3 & 579.8 & 0.2784 \\
0.4 & 577.5 & 0.2713 \\
0.5 & 574.8 & 0.2625 \\
0.6 & 571.9 & 0.2524 \\
0.7 & 569.0 & 0.2411 \\
0.8 & 566.4 & 0.2290 \\
$\mathbf{0 . 9}$ & $\mathbf{5 6 4 . 5}$ & $\mathbf{0 . 2 1 6 4}$ \\
\hline
\end{tabular}



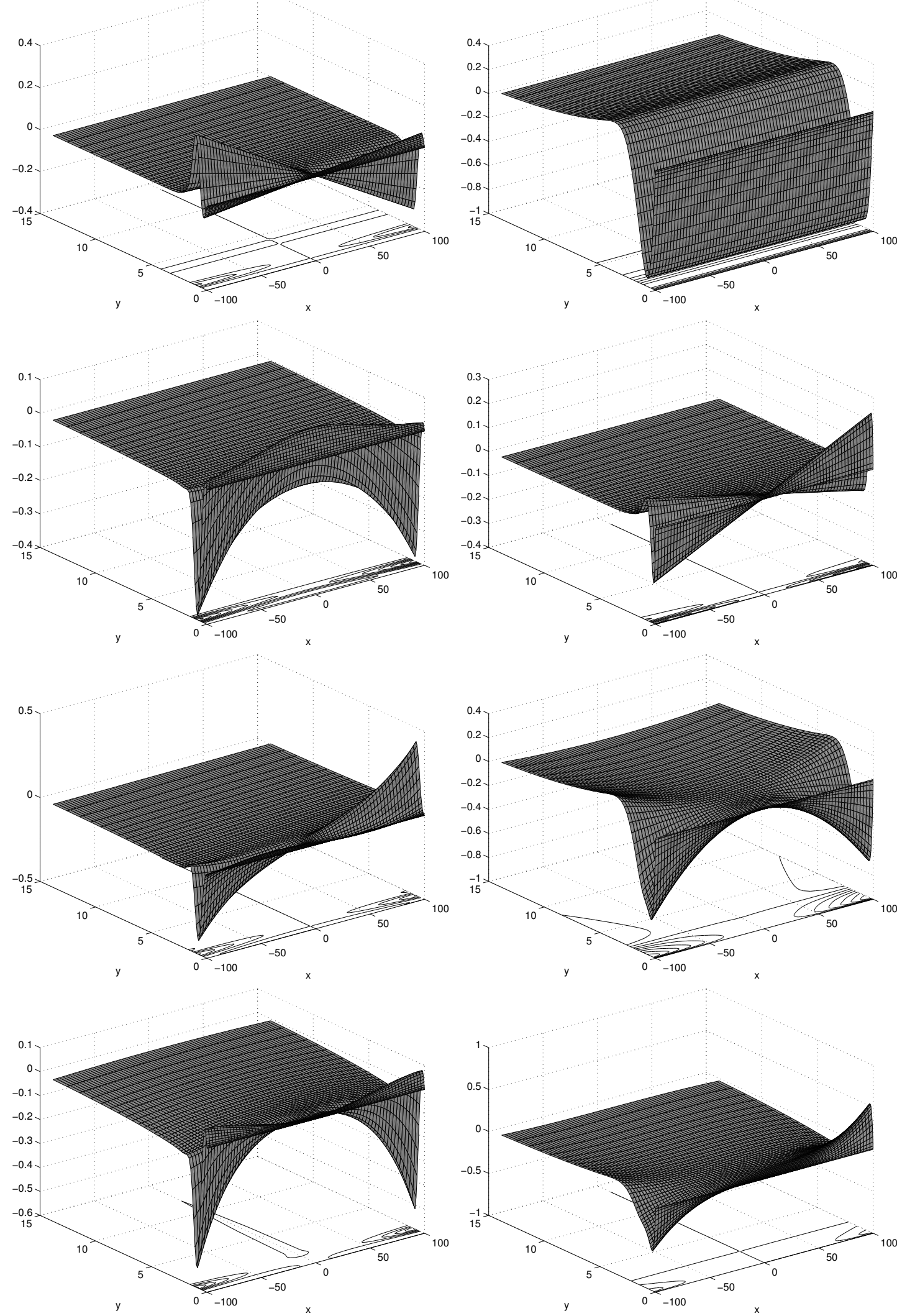

Figura 5.10: Parte real da autofunção em $M a=0.9, R e=800$ e $\beta=0.19$ correspondente aos modos GH ( $1^{a}$ linha), A1 ( $2^{a}$ linha), S2 ( $3^{a}$ linha) e A2 (4 ${ }^{a}$ linha). Coluna esquerda: $u$-velocidade, Coluna direita: $z$-velocidade. 


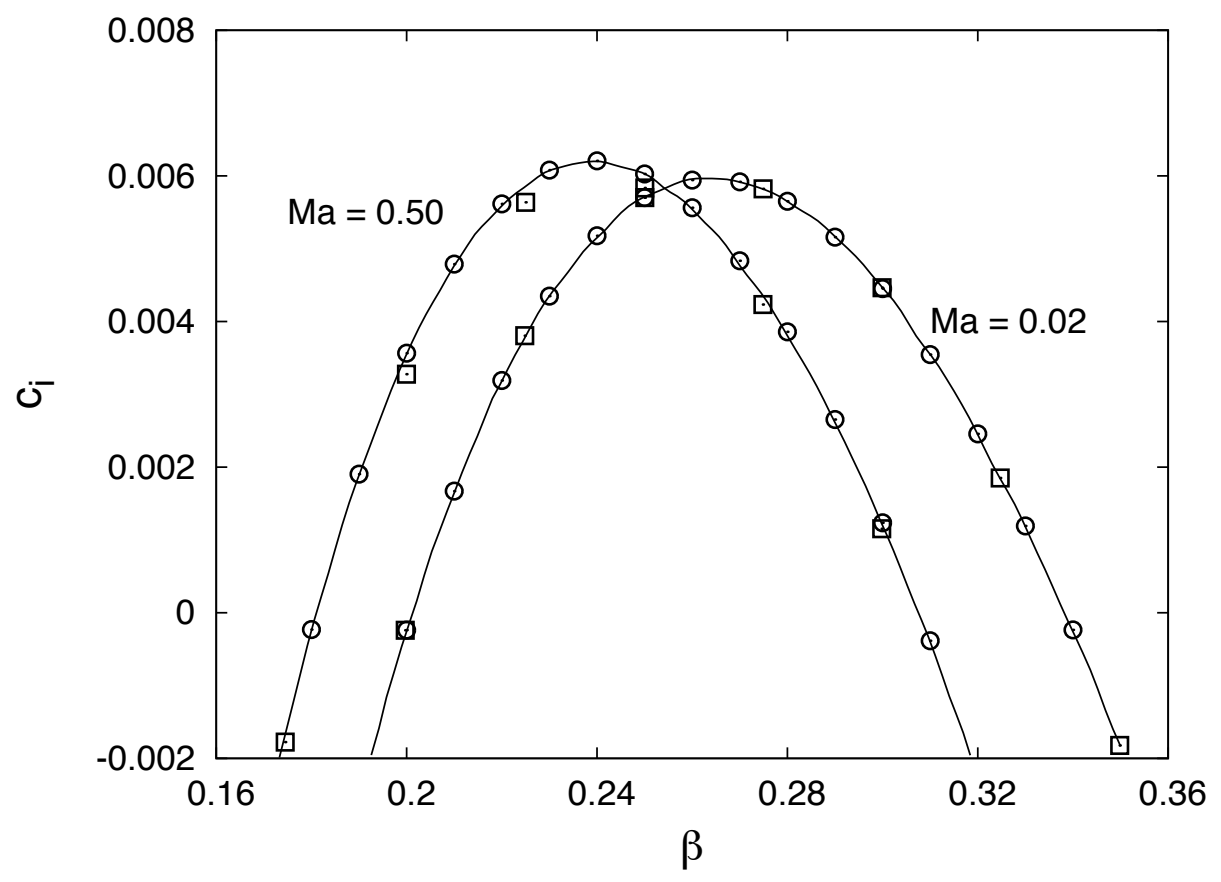

(a) $M a=0.50$ e $M a=0.02$

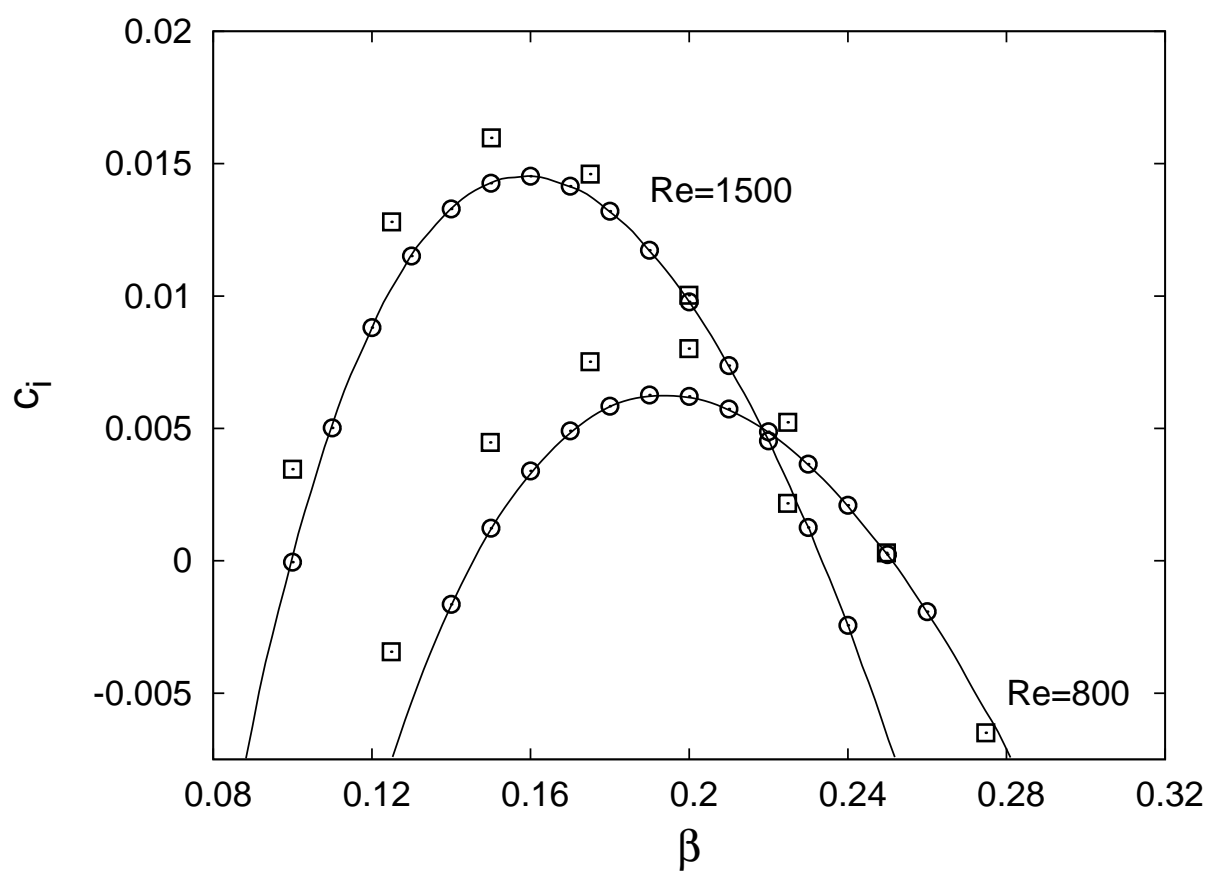

(b) $M a=0.90$

Figura 5.11: Dependência de $c_{i}$ em relação a $\beta$ para o modo GH em (a) $M a=0.5,0.02$ e $R e=800$ (b) $M a=0.9, R e=800$ e $R e=1500$. Linhas sólidas foram obtidas da análise assintótica e símbolos quadrados pela análise BiGlobal de (THEOFILIS; FEDOROV; COLLIS, 2004). Símbolos círculos são resultados do presente trabalho. 


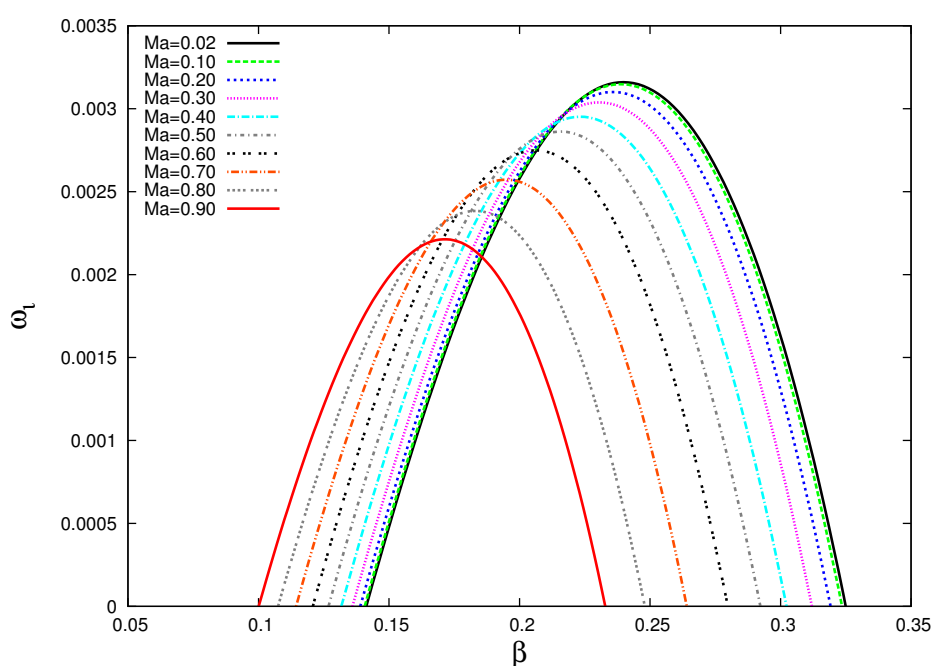

(a)

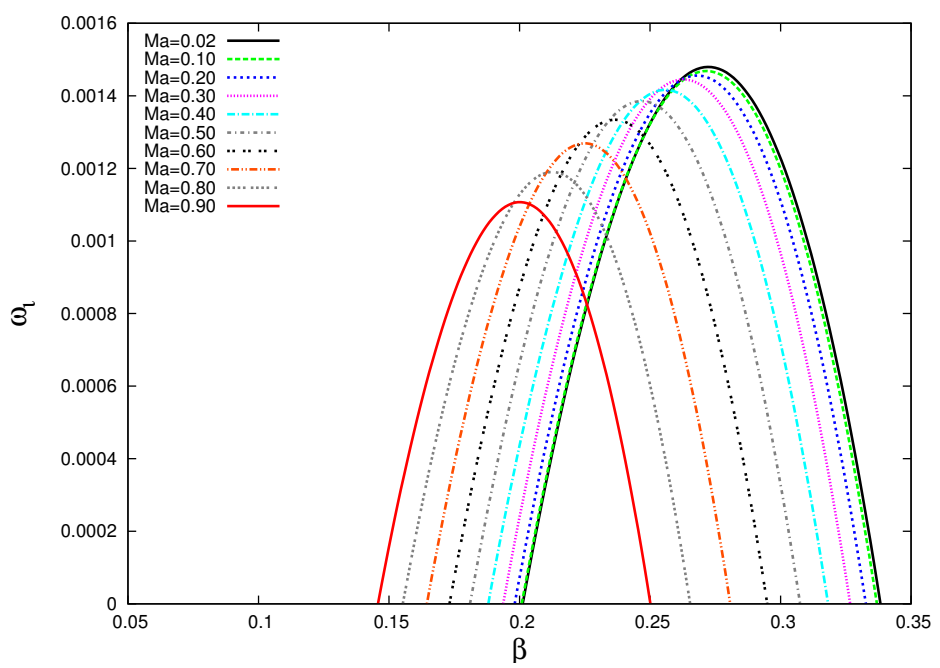

(b)

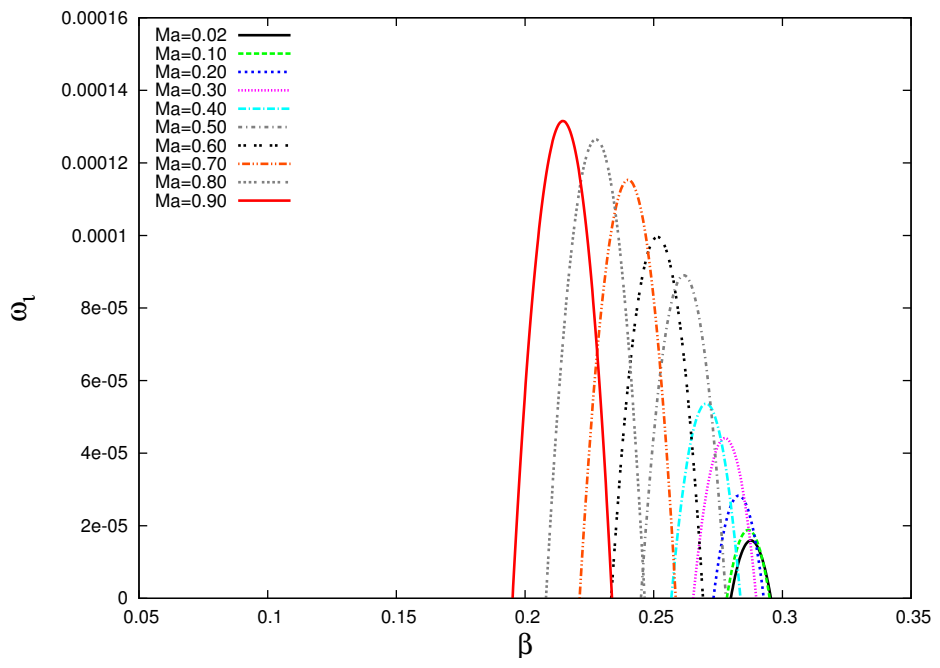

(c)

Figura 5.12: Taxa de amplificação temporal como função de $\beta$ em (a) $\operatorname{Re}=1500$, (b) $\operatorname{Re}=800$ e (c) $\operatorname{Re}=585$. 


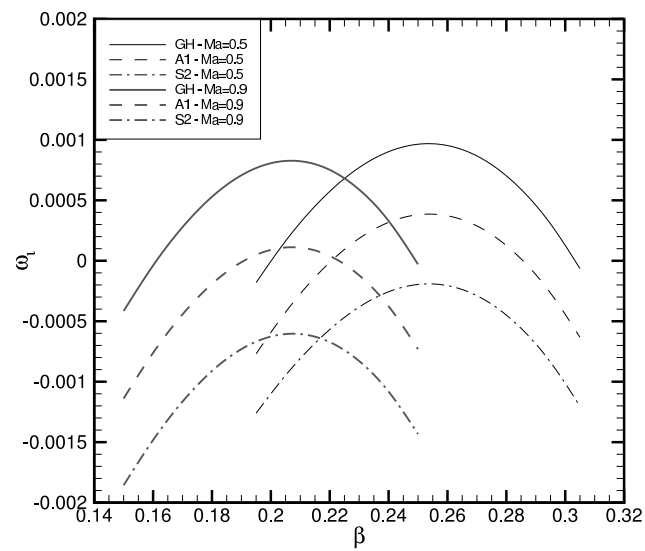

(a) $R e=700$

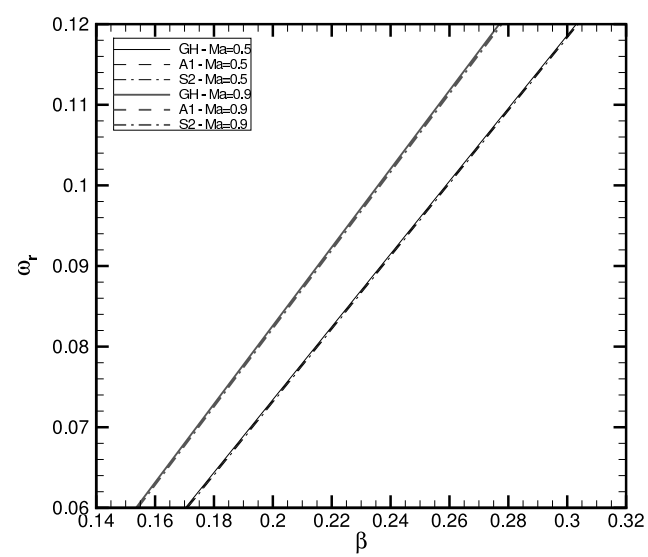

(c) $\operatorname{Re}=700$

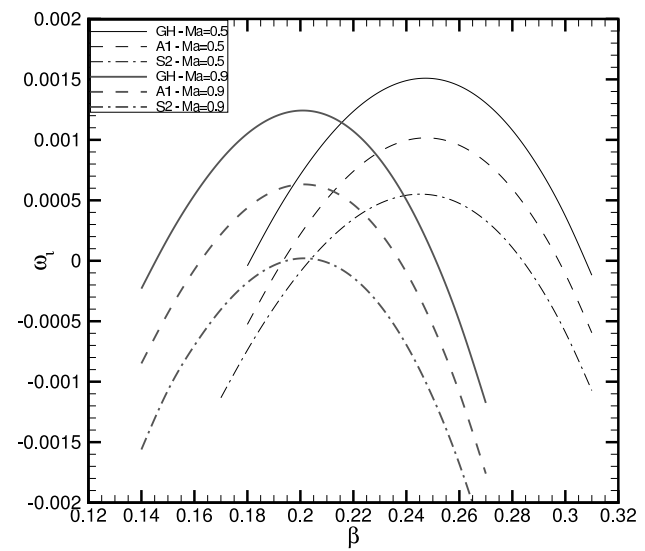

(b) $R e=800$

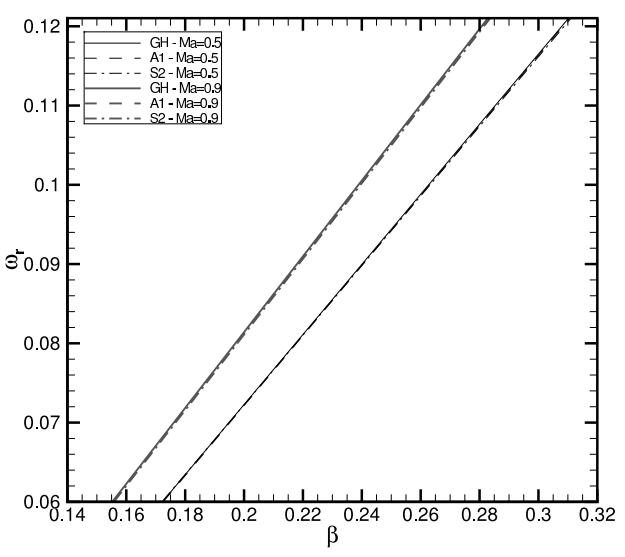

(d) $R e=800$

Figura 5.13: Dependência da taxa de amplificação temporal (a)-(b) e frequência (c)-(d) dos modos GH, A1 e S2 com $\beta$ para $M a=0.50$ e $M a=0.90$. 


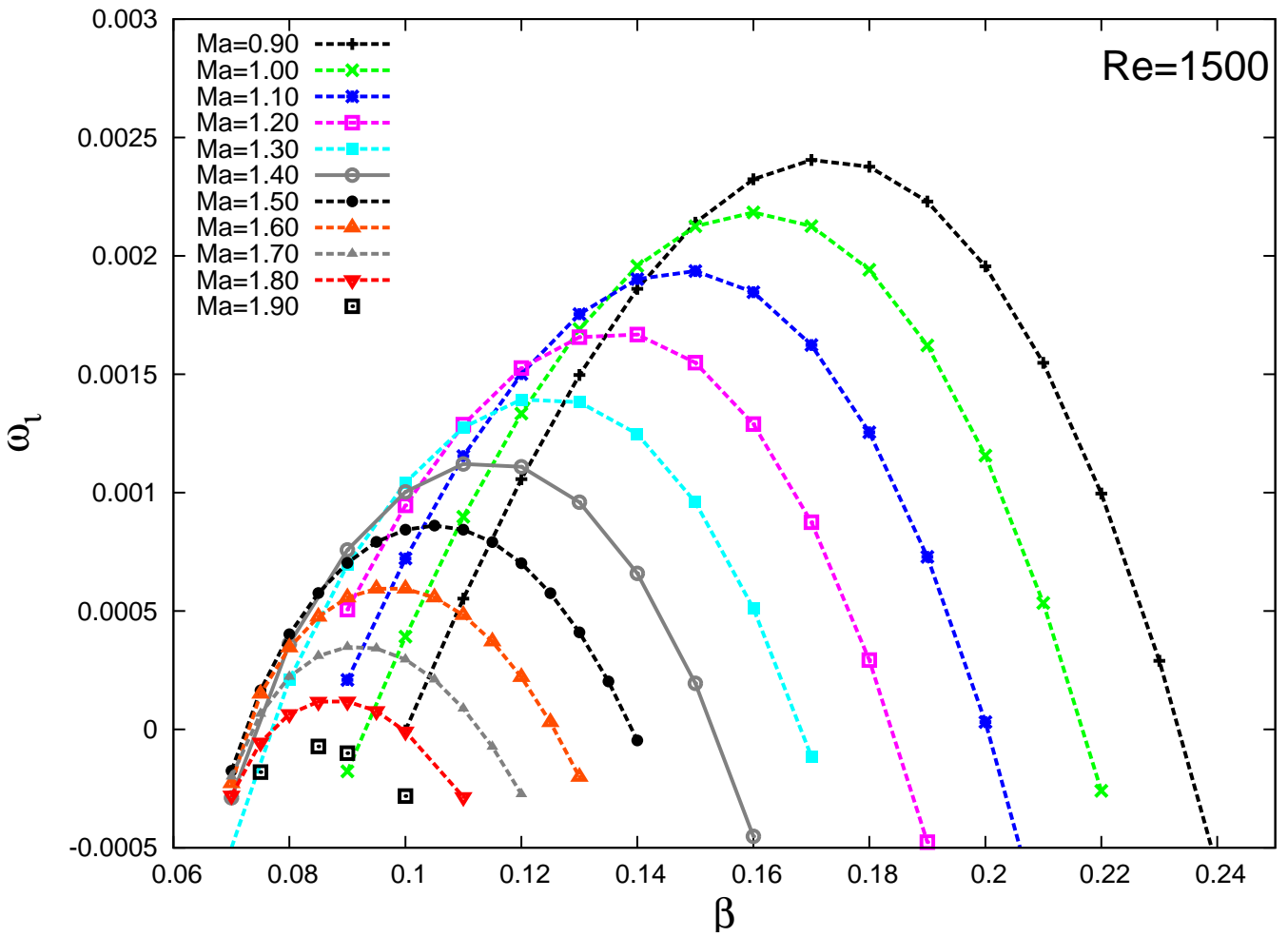

Figura 5.14: Distribuição de $\omega_{i}$ calculado em $R e=1500$ para uma ampla banda de número de onda $\beta$ e uma faixa de número de Mach de $0.02-1.90$.

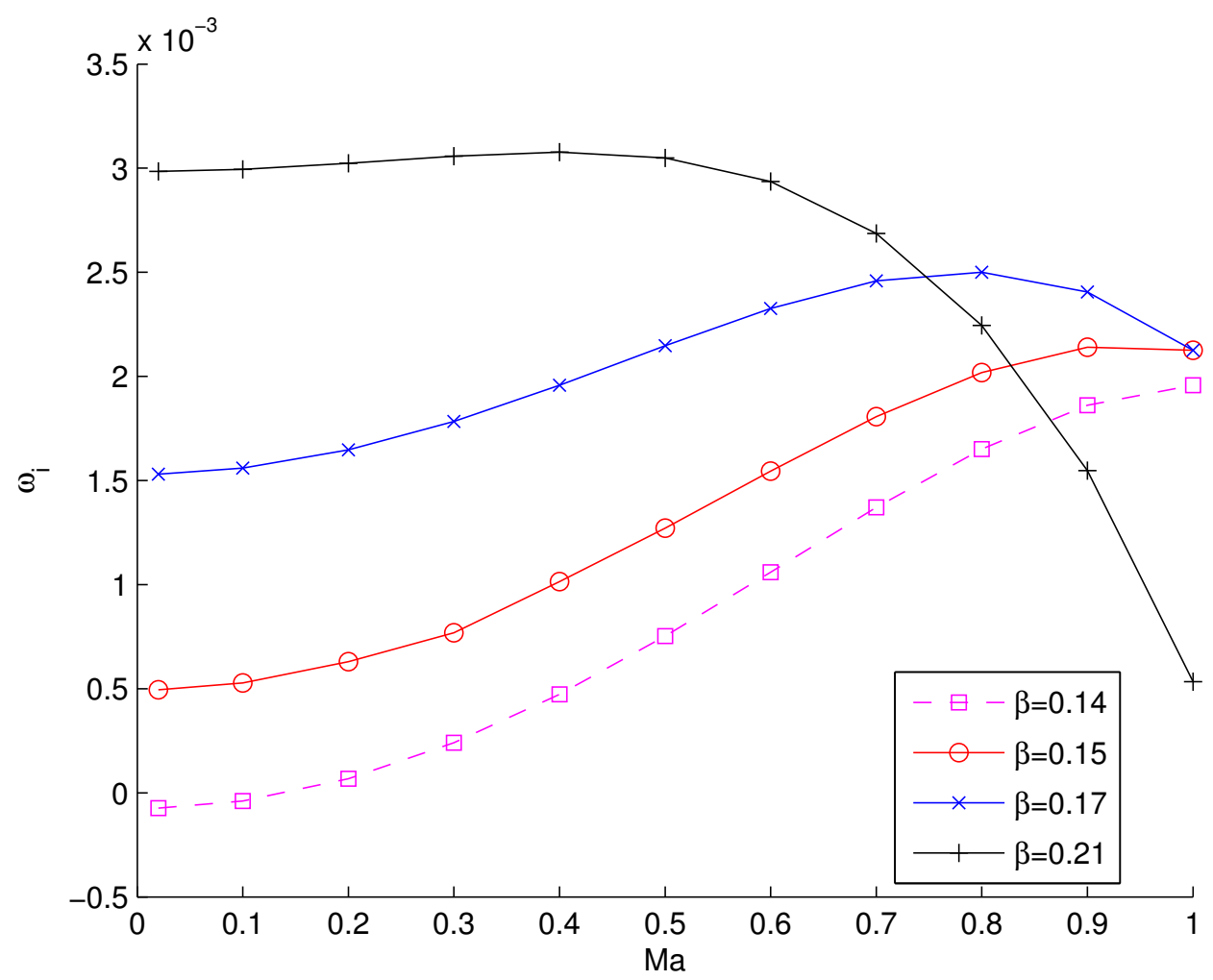

Figura 5.15: Taxa de amplificação temporal correspondente à uma faixa de número de Mach, $R e=1500$ e número de onda fixado. 


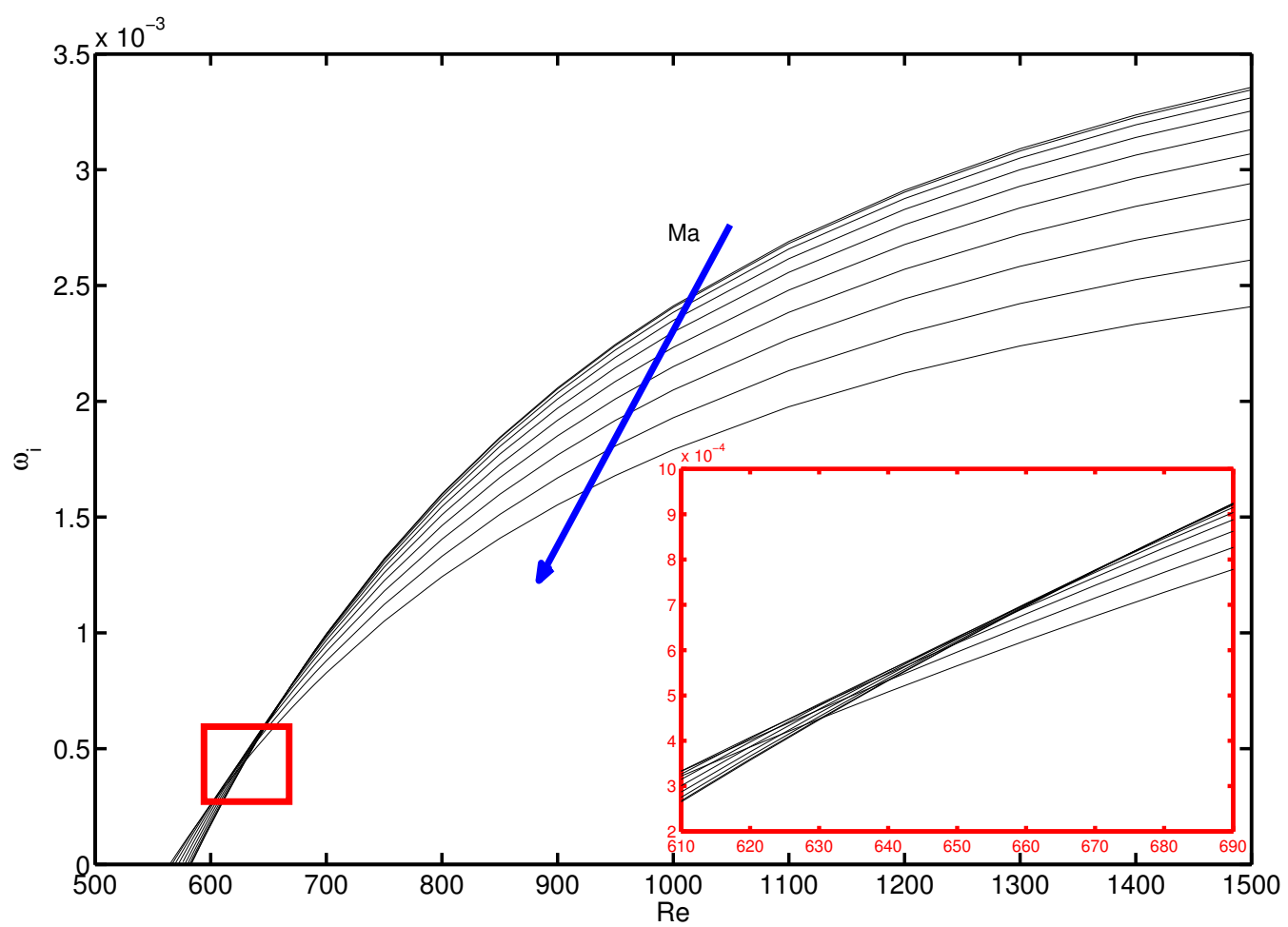

Figura 5.16: Taxa de amplificação temporal $\omega_{i}$ correspondente ao número de onda $\beta$ que mais amplifica como função de número de Reynolds. As linhas correspondem a $M a=\{0.02,0.2,(0.1), 0.9\}$ na direção da seta.

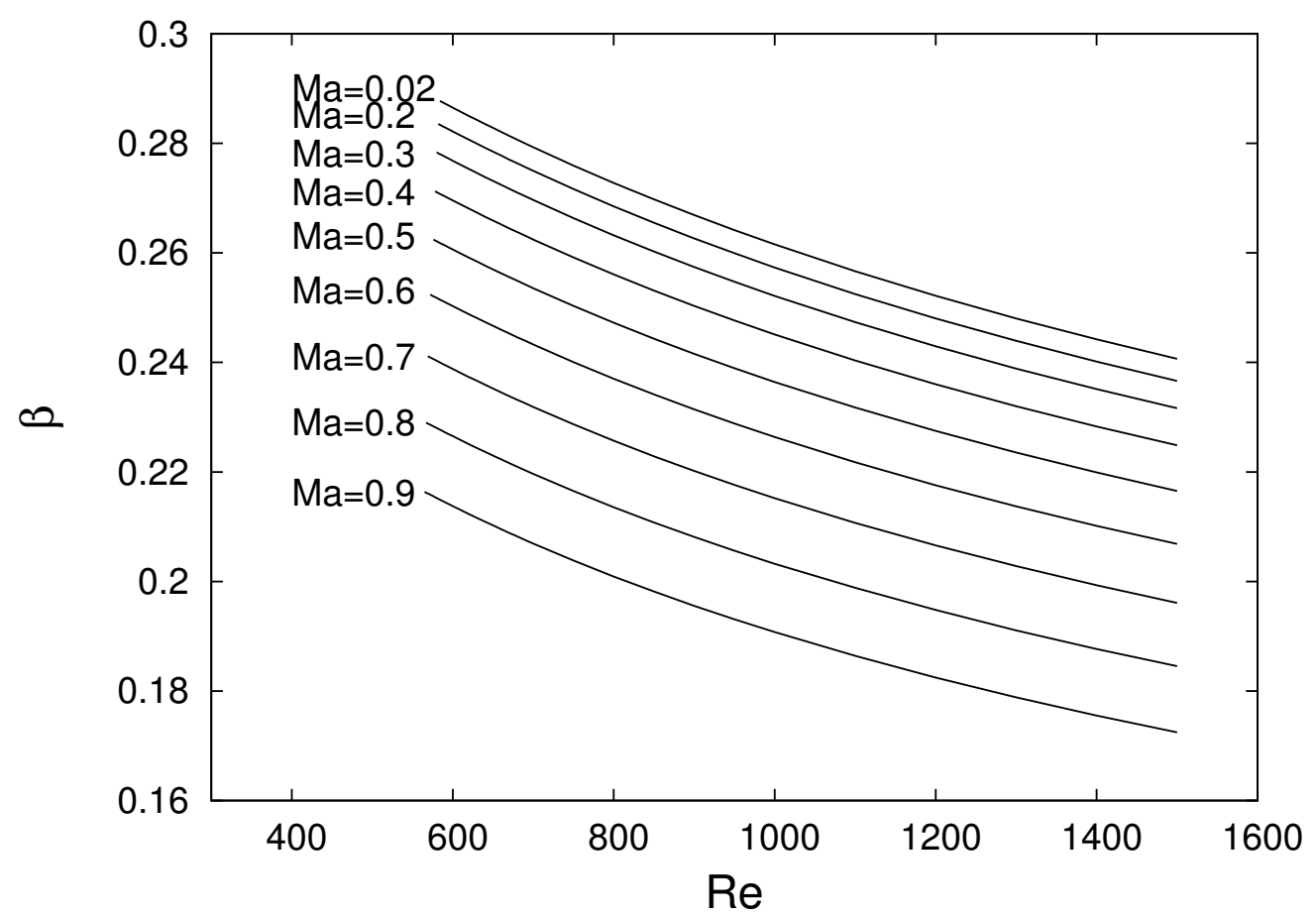

Figura 5.17: Número de onda correspondente a máxima taxa de amplificação temporal versus número de Reynolds. 

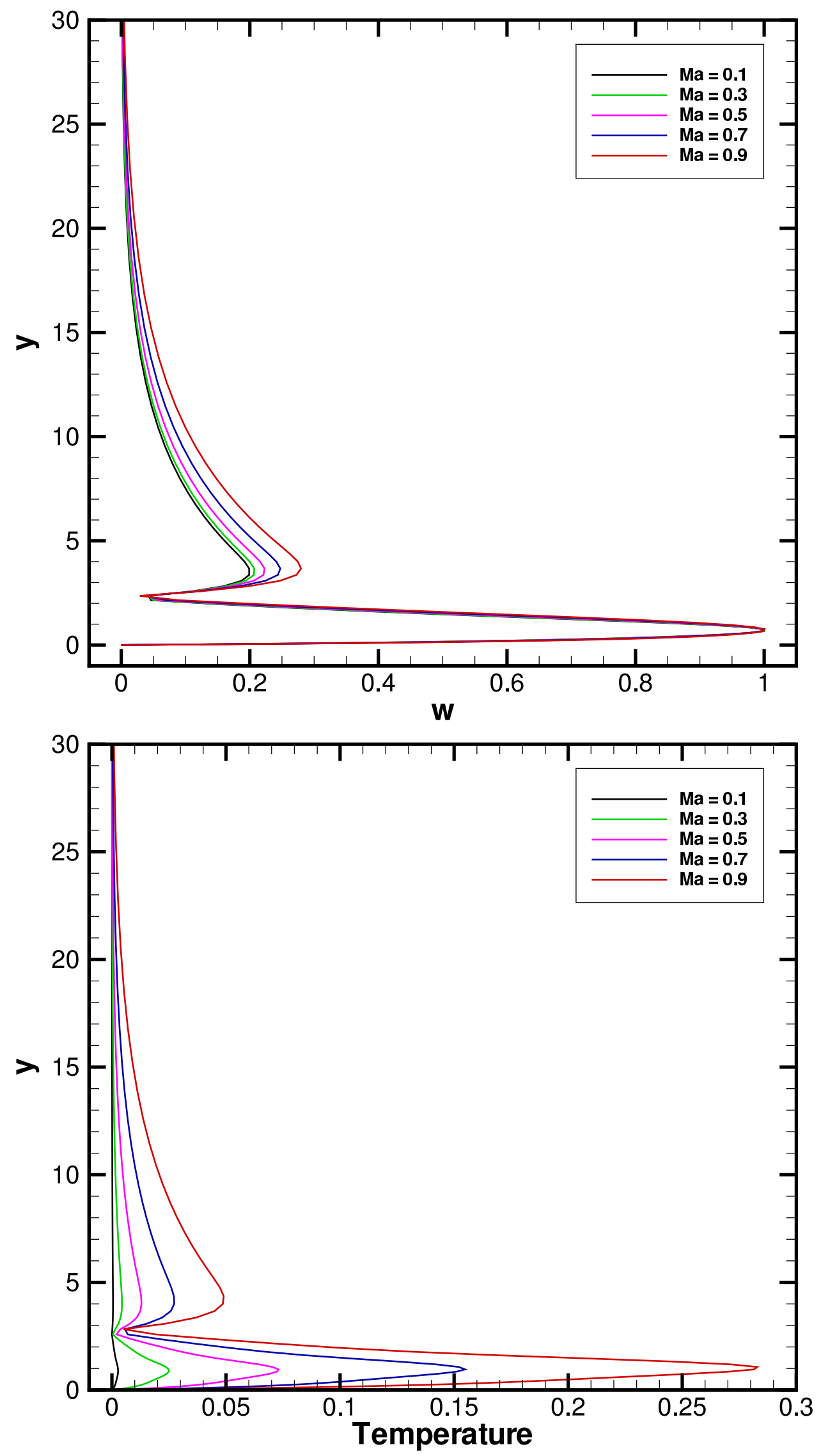

Figura 5.18: Perfis normal à parede para velocidade $w$ (direta) e temperatura (esquerda) do modo dominante GH, como função de $M a=0.9, R e=800$ e $\beta=0.19$. 


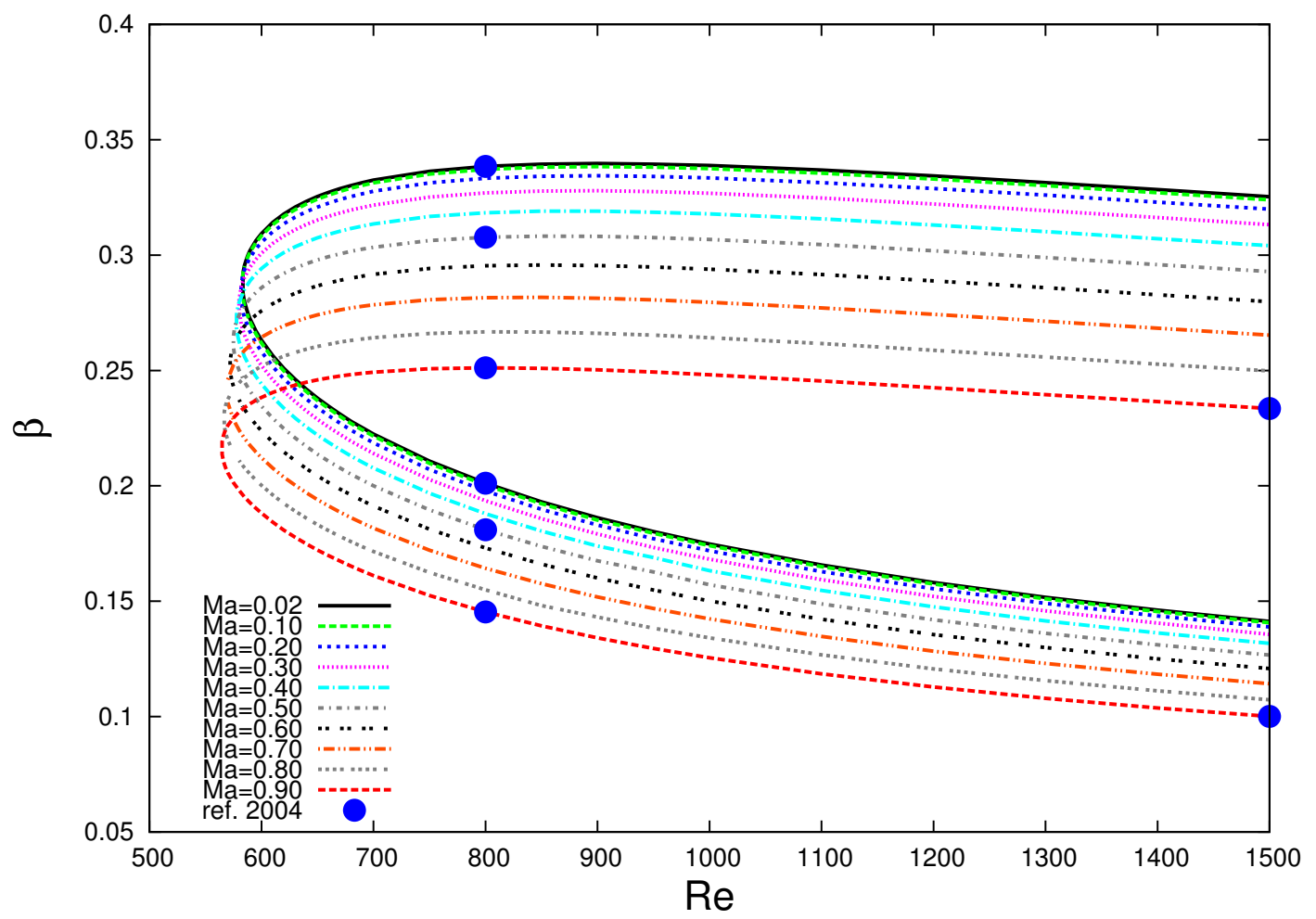

Figura 5.19: Curvas de estabilidade neutra para uma camada limite compressível de uma linha de estagnação de uma asa enflechada obtidas através da análise BiGlobal. Símbolos representam resultados anteriores apresentados por (THEOFILIS; FEDOROV; COLLIS, 2004).

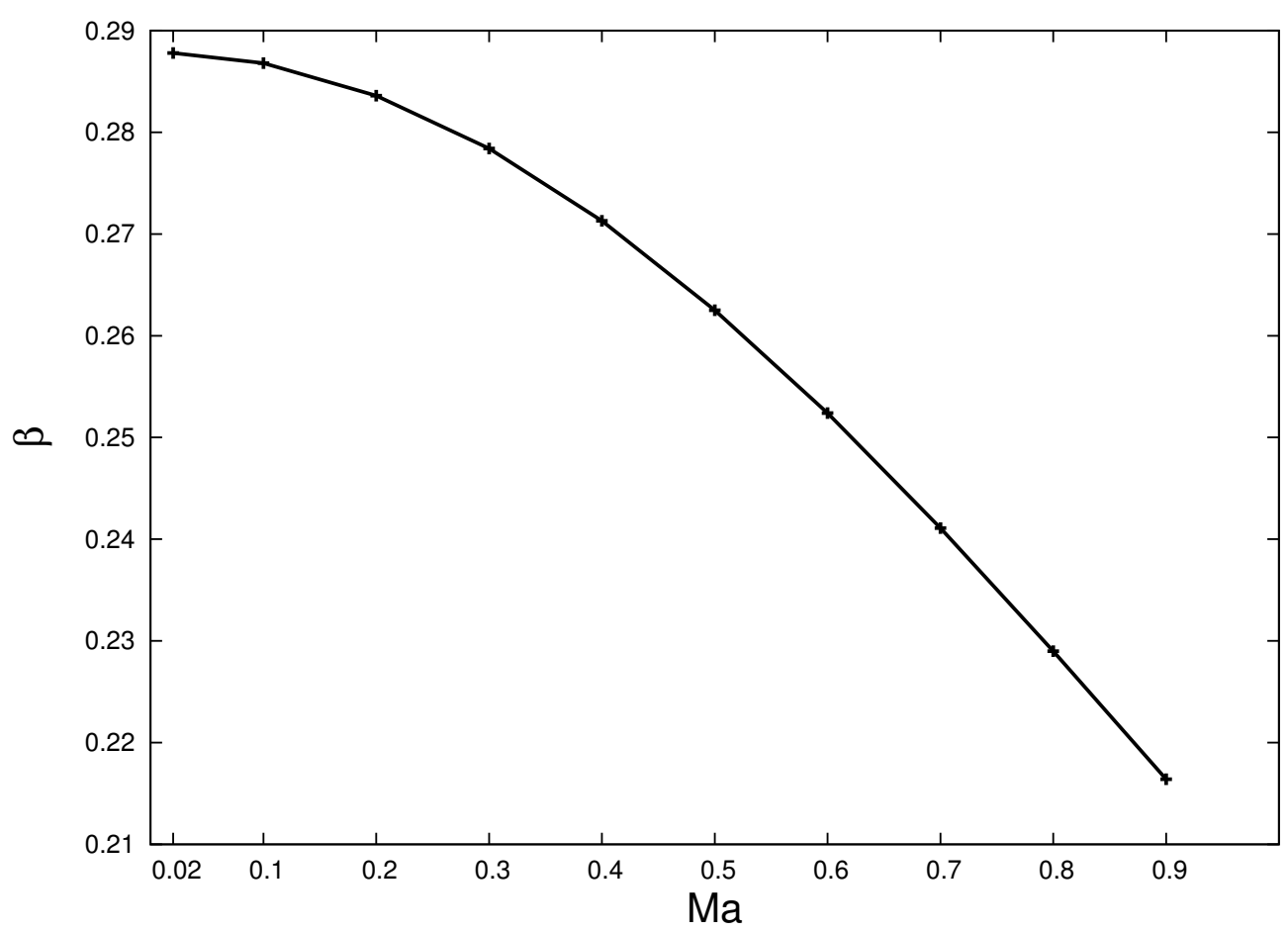

Figura 5.20: Número de onda $\beta$ crítico correspondente a cada número de Mach calculado dentro da faixa subsônica. 


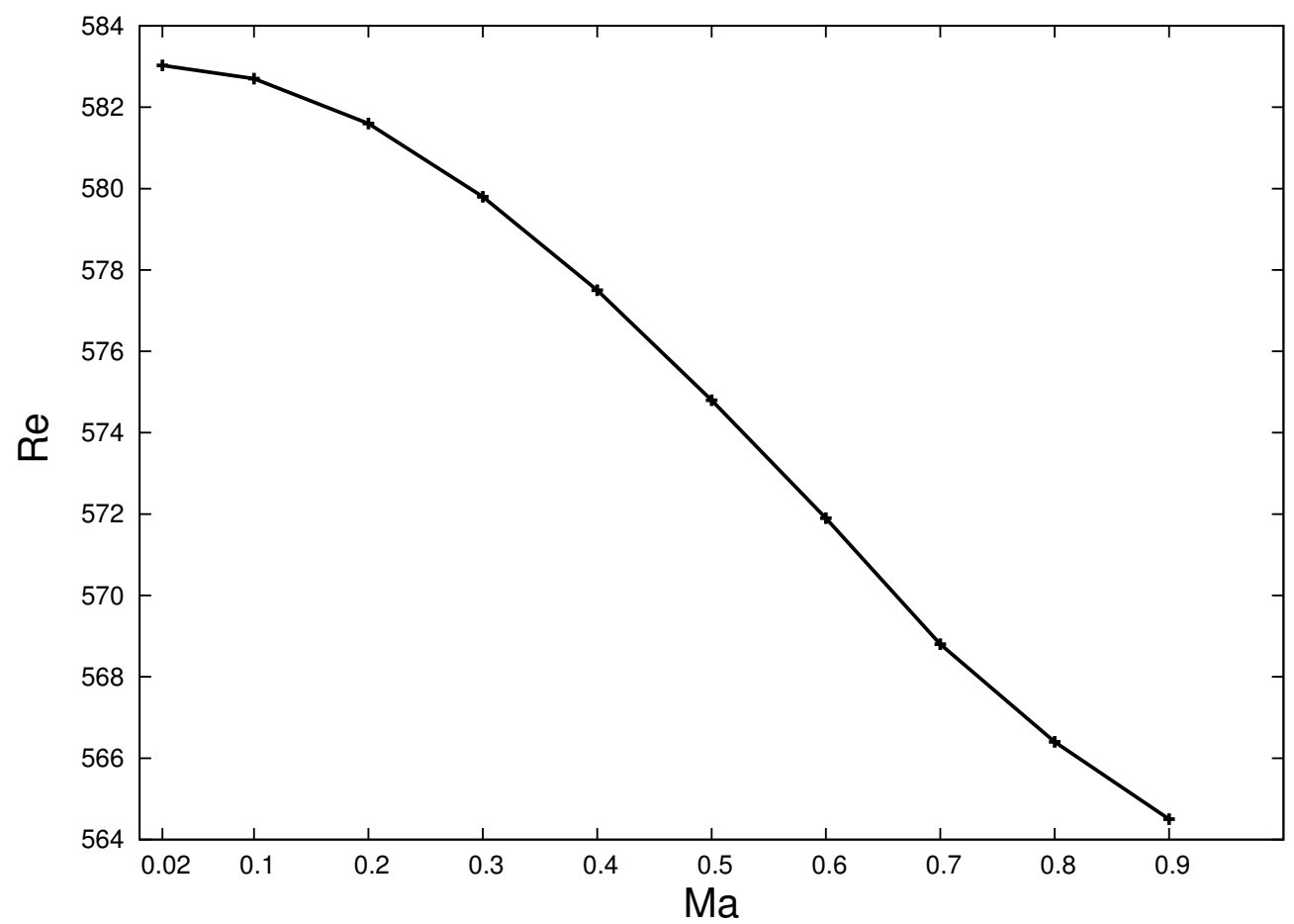

Figura 5.21: Valores extraídos das curvas neutras nas condições críticas para números de Reynolds em diferentes números de Mach. 


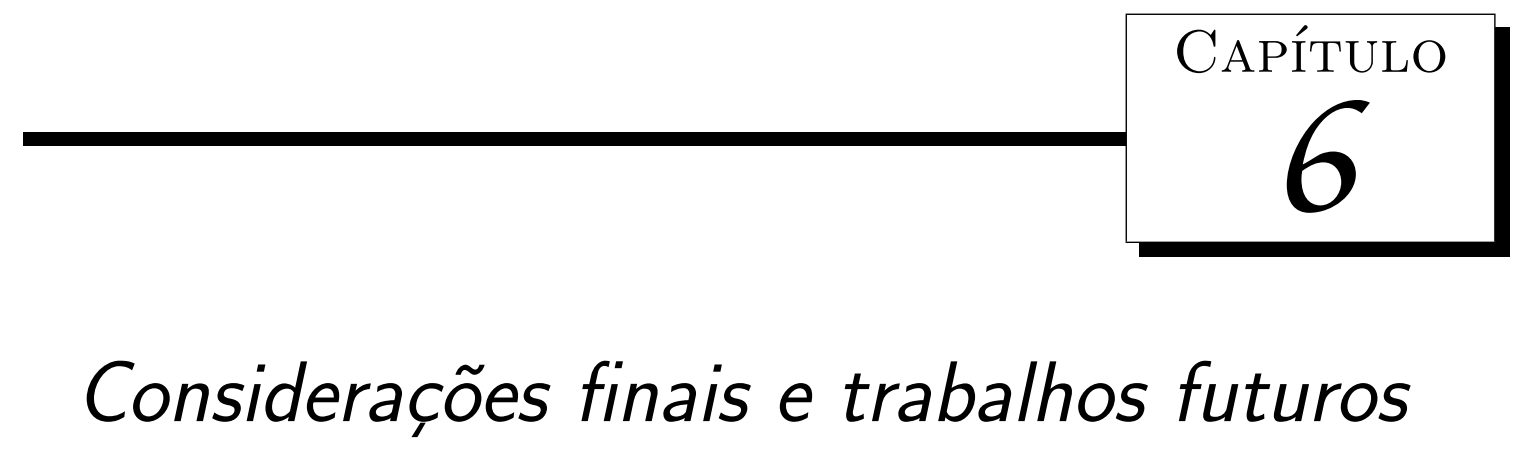

Um novo algoritmo para a solução numérica do problema de autovalor associado à análise da instabilidade linear BiGlobal compressível foi desenvolvido. O algoritmo baseou-se na esparsidade das matrizes resultantes da discretização espacial do problema de autovalor, e teve como um dos principais objetivos a melhoria do desempenho em termos de memória e tempo de CPU em relação às soluções anteriores que utilizaram álgebra densa (RODRÍGUEZ; THEOFILIS, 2009).

Os métodos de discretização espacial implementados foram: Método colocação espectral (pontos de Chebsyhev-Gauss-Lobatto) e esquemas de diferenças finitas de alta ordem. Um estudo do desempenho foi realizado a fim de determinar a solução mais eficiente para um problema físico geral com técnicas de matrizes esparsas. Embora a utilização de um método de colocação espectral nas duas direções levou à melhorias modestas no seu desempenho, uma melhoria notável pode ser visto quando as diferenças finitas foram empregadas para, pelo menos, uma direção espacial.

Para um problema geral físico em que a solução nas duas direções espaciais e são semelhantes e que foram resolvidas, a melhor solução encontrada foi uma discretização usando diferenças finitas de alta ordem nas duas direções. Entretanto, os resultados aqui apresentados demonstraram que, para resolver um problema determinado, com a mesma precisão, para esta combinação foi necessário 1/5-ésima parte da memória e menos do que 
10\% do tempo requerido para a solução através de álgebra densa e métodos espectrais. A partir do estudo do desempenho do código computacional, estimativas para a memória e os requisitos de tempo da CPU foram obtidos para os cálculos de resolução mais elevada. À medida que a resolução desejada foi sendo incrementada, as reduções relativas aos recursos obtidos com a presente metodologia melhoraram. Além disso, quando o problema físico de interesse foi tal que, uma direção espacial apresentasse requisitos de resolução mais exigentes do que a outra, uma combinação dos métodos de colocação espectral e diferenças finitas para discretização espacial melhoraram ainda mais o desempenho.

O código desenvolvido foi então utilizado, a fim de rever e completar um estudo paramétrico sobre a instabilidade de uma camada limite de uma linha de estagnação compressível iniciado por Theofilis, Fedorov e Collis (2004). A referência apresenta uma discrepância entre as previsões de uma teoria assintótica com aquelas fornecidas pela análise BiGlobal, os atuais resultados combinaram perfeitamente com os da teoria assintótica. A resolução limitada devido à versão álgebra densa do algoritmo usado em (THEOFILIS; FEDOROV; COLLIS, 2004) pode ser considerada como insuficiente para convergência dos resultados, motivo pelo qual não houve concordância entre os resultados.

Este trabalho apresentou um estudo completo paramétrico da instabilidade de perturbações modais tri-dimensionais em regime subsônico do escoamento de uma camada limite de uma linha de estagnação. Verificou-se que, para número de Reynolds, $R e$, relativamente alto, o decremento do número de Mach resultou em um aumento tanto da faixa de números de onda $\beta$ instáveis como a taxa de amplificação máxima para um número de Reynolds dado, um resultado que está de acordo com a maioria dos resultados na literatura a respeito instabilidade viscoso de escoamentos compressíveis. A taxa de crescimento aumentou monotonicamente com o número de Reynolds para a banda de $M a$ investigada. No entanto, encontrou-se também que o número de Reynolds crítico diminui com o aumento do número Mach. Embora este decréscimo tenha sido muito modesto, que implica que o escoamento tornou-se instável, mais cedo (em termos de número de Reynolds), que pode ter importantes implicações do ponto de vista da engenharia. Esta 
tendência parece estar associada com o aumento da viscosidade das ondas de instabilidade por conta das flutuações de temperatura mais elevada à medida que a compressibilidade aumenta.

Investigações das intabilidades do escomento em regime supersônico podem ser uma sequência natural deste trabalho e os efeitos do crescimento transiente. Do ponto de vista numérico, outros aspectos podem ser investigados visando o melhor aproveitamento dos recursos computacionais. 


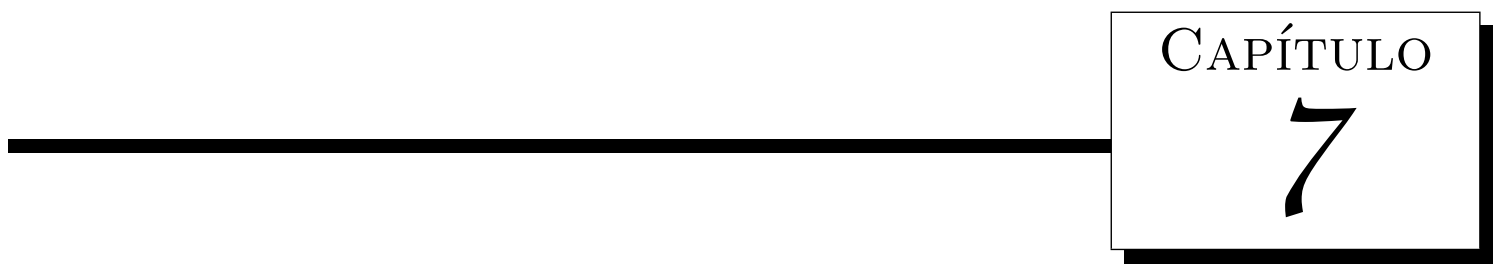

\section{Apêndice}

\subsection{Operador compressível linearizado}

Em alguma situação, quando o escoamento básico é estabelecido, as esquações de Navier-Stokes podem ser escritas em termos das variáveis perturbação e linearizadas sobre o escoamento base para estudar o desenvolvimento de pequenas perturbações ao redor deste escoamento básico. Se o mesmo depende de duas direções espaciais não homogêneas, a perturbação pode ser considerada periódica ao longo da direção espacial homogênea e então introduz-se os modos de Fourier. Uma dependência exponencial é assumido no tempo para as perturbações e consequentemente tem-se um problema de autovalor generalizado bidimensional, onde os autovalores são frequências complexas e as autofunções dependem das duas direções espaciais não homogêneas. Esta abordagem foi descrita anteriormente em detalhes e nesta seção apresentamos todos os termos das equações de Navier-Stokes 
linearizadas, continuidade e energia.

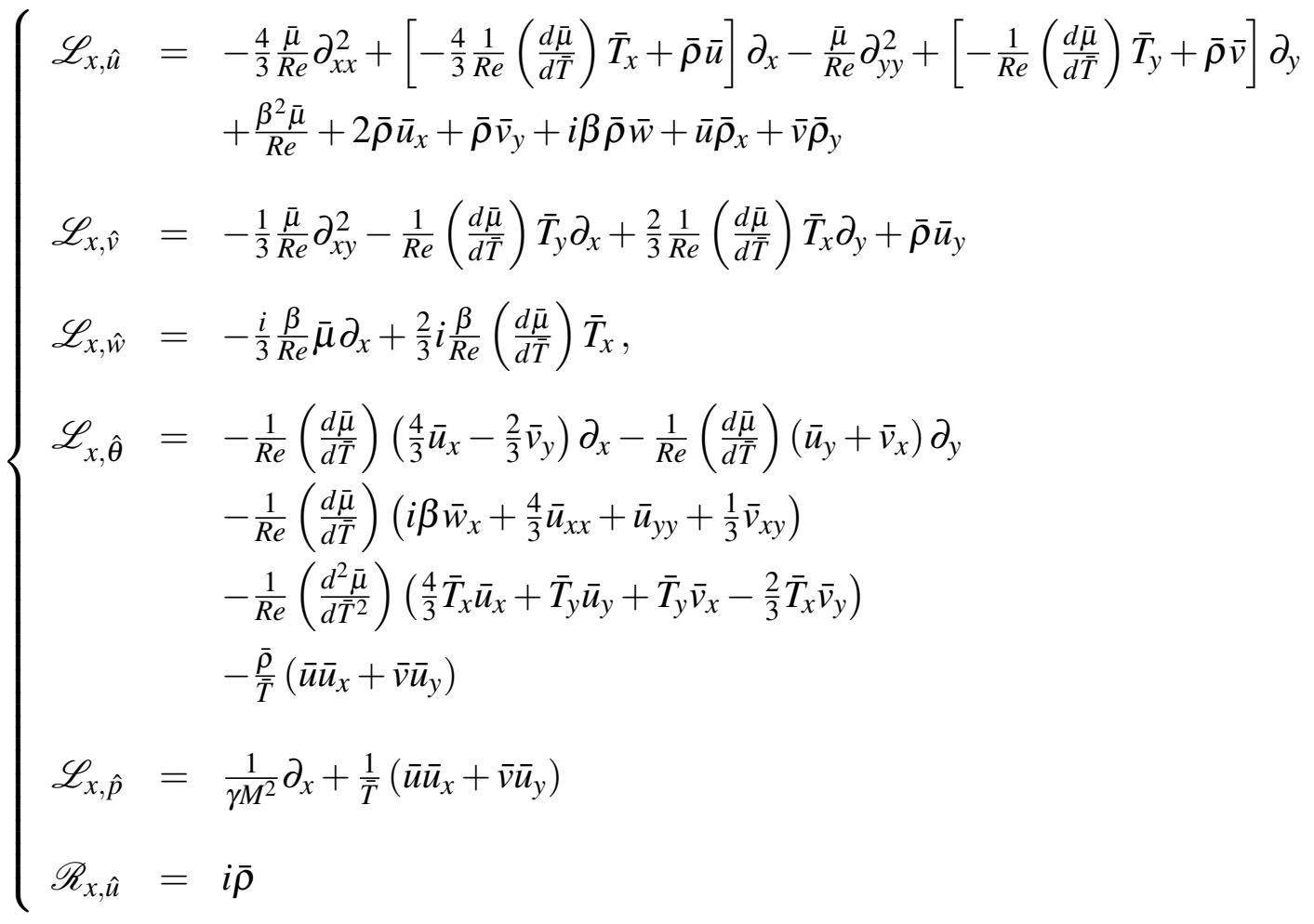

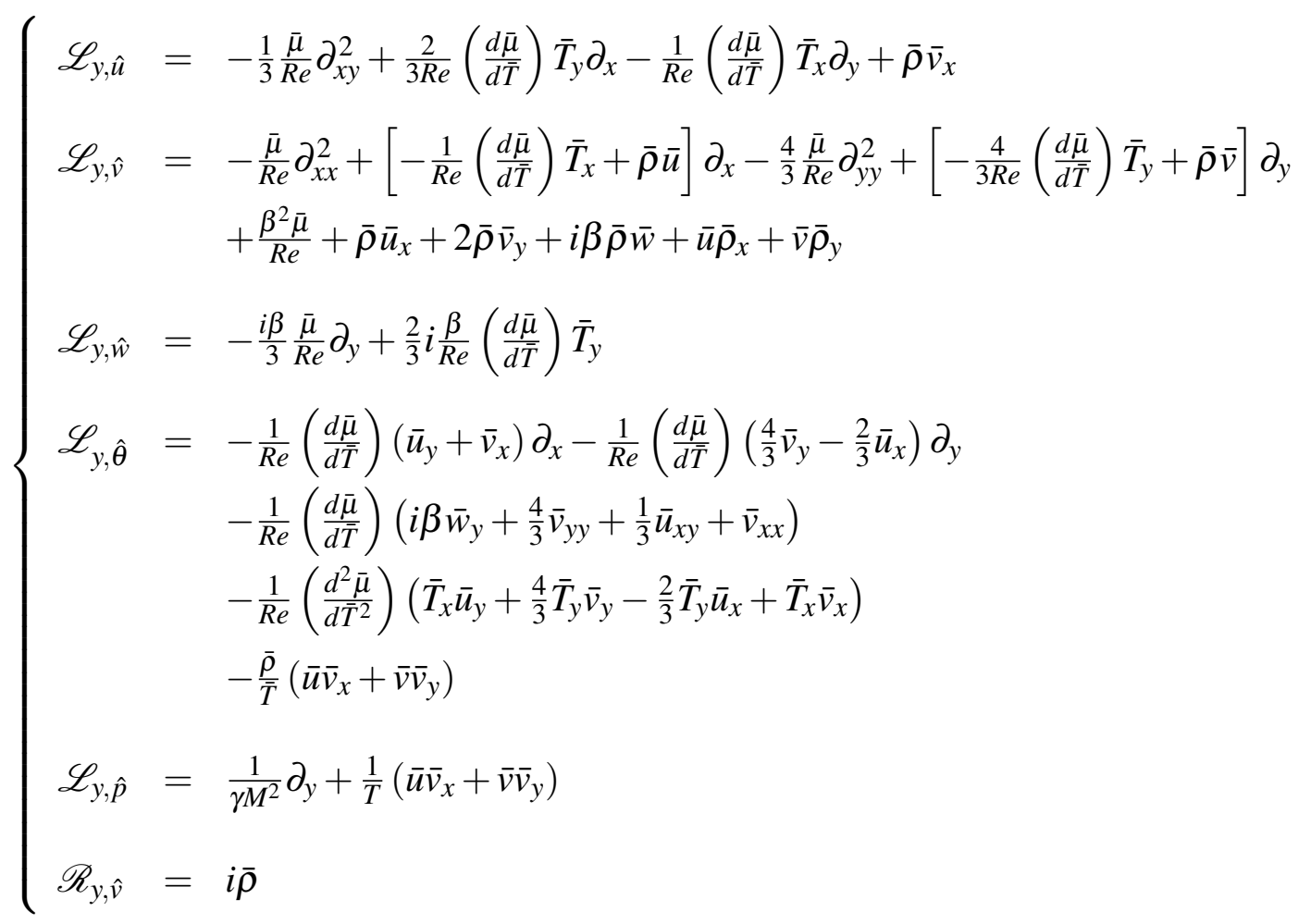




$$
\begin{aligned}
& \left\{\begin{aligned}
\mathscr{L}_{z, \hat{u}}= & -\frac{i \beta}{3} \frac{\bar{\mu}}{R e} \partial_{x}-\frac{i \beta}{R e}\left(\frac{d \bar{\mu}}{d \bar{T}}\right) \bar{T}_{x}+\bar{\rho} \bar{w}_{x} \\
\mathscr{L}_{z, \hat{v}}= & -\frac{i \beta}{3} \frac{\bar{\mu}}{R e} \partial_{y}-\frac{i \beta}{R e}\left(\frac{d \bar{\mu}}{d \bar{T}}\right) \bar{T}_{y}+\bar{\rho} \bar{w}_{y} \\
\mathscr{L}_{z, \hat{w}}= & -\frac{\bar{\mu}}{R e} \partial_{x x}^{2}+-\frac{\bar{\mu}}{R e} \partial_{y y}^{2}+\left[-\frac{1}{R e}\left(\frac{d \bar{\mu}}{d \bar{T}}\right)\left(\bar{u}_{y}+\bar{v}_{x}\right)\right] \partial_{x}+\left[-\frac{1}{R e}\left(\frac{d \bar{\mu}}{d \bar{T}}\right) \bar{T}_{y}+\bar{\rho} \bar{v}\right] \partial_{y} \\
& +\frac{4 \bar{\mu}}{3 R e} \beta^{2}+\bar{\rho} \bar{u}_{x}+\bar{\rho} \bar{v}_{y}+i \beta \bar{\rho} \bar{w}+\bar{u} \bar{\rho}_{x}+\bar{v} \bar{\rho}_{y} \\
\mathscr{L}_{z, \hat{\theta}}= & -\frac{1}{R e}\left(\frac{d \bar{\mu}}{d \bar{T}}\right) \bar{w}_{x} \partial_{x}-\frac{1}{R e}\left(\frac{d \bar{\mu}}{d \bar{T}}\right) \bar{w}_{y} \partial_{y}-\frac{1}{R e}\left(\frac{d \bar{\mu}}{d \bar{T}}\right)\left[\bar{w}_{x x}+\bar{w}_{y y}-\frac{2}{3} i \beta\left(\bar{u}_{x}+\bar{v}_{y}\right)\right] \\
& -\frac{1}{R e}\left(\frac{d^{2} \bar{\mu}}{d \bar{T}^{2}}\right)\left[\bar{T}_{x} \bar{w}_{x}+\bar{T}_{y} \bar{w}_{y}\right]-\frac{\bar{\rho}}{\bar{T}}\left(\bar{u} \bar{w}_{x}+\bar{v} \bar{w}_{y}\right) \\
\mathscr{L}_{z, \hat{p}}= & \frac{i \beta}{\gamma M^{2}}+\frac{1}{\bar{T}}\left(\bar{u} \bar{w}_{x}+\bar{v} \bar{w}_{y}\right) \\
\mathscr{R}_{z, \hat{w}}= & i \bar{\rho}
\end{aligned}\right.
\end{aligned}
$$

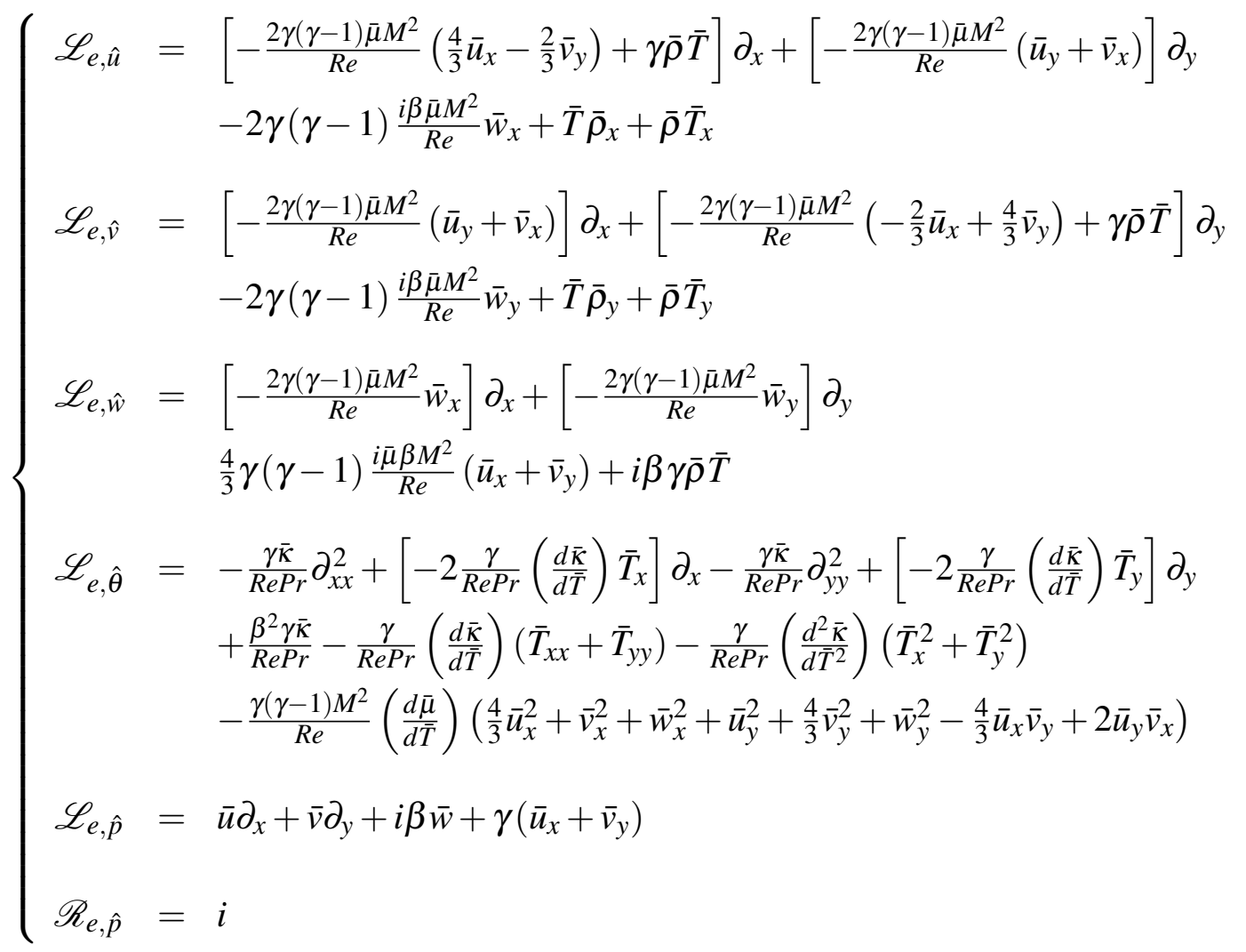




$$
\left\{\begin{array}{l}
\mathscr{L}_{c, \hat{u}}=\bar{\rho} \bar{T} \partial_{x}+\bar{T} \bar{\rho}_{x} \\
\mathscr{L}_{c, \hat{v}}=\bar{\rho} \bar{T} \partial_{y}+\bar{T} \bar{\rho}_{y} \\
\mathscr{L}_{c, \hat{w}}=i \beta \bar{\rho} \bar{T} \\
\mathscr{L}_{c, \hat{\theta}}=-\bar{\rho} \bar{u} \partial_{x}-\bar{\rho} \bar{v} \partial_{y}-\bar{\rho} \bar{u}_{x}-\bar{\rho} \bar{v}_{y}-i \beta \bar{\rho} \bar{w}-\bar{u} \bar{\rho}_{x}-\bar{v} \bar{\rho}_{y}+\frac{\bar{\rho}}{\bar{T}}\left(\bar{u} \bar{T}_{x}+\bar{v} \bar{T}_{y}\right) \\
\mathscr{L}_{c, \hat{p}}^{G}=\bar{u} \partial_{x}+\bar{v} \partial_{y}+i \beta \bar{w}+\bar{u}_{x}+\bar{v}_{y}-\frac{1}{\bar{T}}\left(\bar{u} \bar{T}_{x}+\bar{v} \bar{T}_{y}\right) \\
\mathscr{R}_{c, \hat{\theta}}=-i \bar{\rho} \\
\mathscr{R}_{c, \hat{p}}=i
\end{array}\right.
$$




\subsection{Descrição método de Arnoldi}

Descreveremos o método de Arnoldi partindo de um sistema linear

$$
A x=b
$$

considerando $A$ matriz não-singular e sendo $x^{*}$ a solução exata.

Seja $q$ o polinômio minimal associado a $A$ tal que $q(A)=0$. Assim

$$
q=\alpha_{0} I+\alpha_{1} A+\ldots+\alpha_{m} A^{m}=0
$$

de modo que $\alpha_{0} \neq 0$ ( $A$ matriz não-singular $)$,

$$
\begin{aligned}
I & =-\frac{1}{\alpha_{0}} \sum_{j=1}^{m} \alpha_{j} A^{j} \\
A^{-1} & =-\frac{1}{\alpha_{0}} \sum_{j=0}^{m-1} \alpha_{j+1} A^{j} .
\end{aligned}
$$

Por outro lado, uma solução exata do sistema linear é dados por:

$$
\begin{aligned}
x^{*} & =A^{-1} b \\
& =\left(-\frac{1}{\alpha_{0}} \sum_{j=0}^{m-1} \alpha_{j+1} A^{j}\right) b
\end{aligned}
$$

Assim, escrevemos $x^{*}$ como uma combinação linear de $\left(b, A b, A^{2} b, \ldots, A^{m-1} b\right)$ e podemos usar o subespaço de Krylov $K_{m}(A, b)$ como o espaço de busca da solução aproximada.

Suponhamos então $x_{0} \neq 0, \log 0$

$$
\begin{aligned}
x^{*} & =A^{-1} b \\
& =A^{-1}\left(A x_{0}+r_{0}\right) \\
& =x_{0}+A^{-1} r_{0}
\end{aligned}
$$

e portanto $x^{*} \in x_{0}+K_{m}\left(A, r_{0}\right)$.

O processo de Arnoldi é um método para computar uma base ortornormal $\left\{v_{1}, v_{2}, \ldots, v_{j}\right\}$ 
do subespaço de Krylov, $K_{j}\left(A, v_{1}\right)$. Ortogonalização foi obtida através de uma procedimento muito conhecido por Gram-Schmidt.

Um subespaço de Krylov é definido por:

$$
K_{m}=\operatorname{span}\left\{\mathbf{q}_{0}, \mathbf{M} \mathbf{q}_{0}, \mathbf{M}^{2} \mathbf{q}_{0}, \ldots, \mathbf{M}^{m-1} \mathbf{q}_{0}\right\}
$$

onde $\mathbf{M}$ é a matriz discretizada e $\mathbf{q}_{0}$ um vetor arbitrário qualquer. 


\subsection{Dados do escoamento base de uma camada limite compressível ao longo do bordo de ataque de uma asa enflechada}

A tabela 7.1 apresenta valores de temperatura e deformação na parede para uma faixa de número de Mach.

Tabela 7.1: Dependência de $U_{0}{ }^{\prime}(0), W_{0}{ }^{\prime}(0)$ e temperatura na parede do escoamento base para $M a \in\left[10^{-5}, 5.00\right]$.

\begin{tabular}{c|ccc}
\hline$M a$ & $U_{0}{ }^{\prime}(0)$ & $W_{0}{ }^{\prime}(0)$ & $T_{0}(0)$ \\
\hline $10^{-5}$ & 1.232588 & 0.570465 & 1.000000 \\
0.02 & 1.232561 & 0.570437 & 1.000069 \\
0.10 & 1.231925 & 0.569760 & 1.001719 \\
0.20 & 1.122995 & 0.567658 & 1.006878 \\
0.25 & 1.228483 & 0.566095 & 1.010746 \\
0.30 & 1.231925 & 0.569760 & 1.001719 \\
0.40 & 1.222256 & 0.559456 & 1.027512 \\
0.50 & 1.216689 & 0.553511 & 1.042990 \\
0.60 & 1.210112 & 0.546474 & 1.061909 \\
0.70 & 1.202641 & 0.538462 & 1.084270 \\
0.75 & 1.198610 & 0.534132 & 1.096741 \\
0.80 & 1.194403 & 0.529606 & 1.110074 \\
0.90 & 1.185527 & 0.520035 & 1.139322 \\
1.00 & 1.176141 & 0.509881 & 1.172016 \\
1.10 & 1.166369 & 0.499270 & 1.208156 \\
1.20 & 1.156326 & 0.488320 & 1.247744 \\
1.30 & 1.146119 & 0.477141 & 1.290779 \\
1.40 & 1.135843 & 0.465832 & 1.337264 \\
1.50 & 1.125582 & 0.454480 & 1.387198 \\
1.60 & 1.115407 & 0.443162 & 1.440583 \\
1.70 & 1.105380 & 0.431942 & 1.497418 \\
1.80 & 1.095549 & 0.420874 & 1.557704 \\
1.90 & 1.085956 & 0.410001 & 1.621441 \\
2.00 & 1.076630 & 0.399360 & 1.688629 \\
3.00 & 1.000514 & 0.308888 & 2.550336 \\
4.00 & 0.952139 & 0.245969 & 3.757095 \\
5.00 & 0.921755 & 0.202362 & 5.308874 \\
\hline & & &
\end{tabular}




\subsection{Formato da matriz esparsa comprimida}

Uma forma de representar uma matriz esparsa de maneira comprimida é escrevê-la no formato CSR. Este formato consiste em descrever a matriz $n \times n$ em três vetores, sendo eles: os valores, colunas e índice das linhas. Não existem restrições no formato CSR.

Para exemplificar, seja a matriz $M$ de dimensão $5 \times 5$ com 13 elementos diferentes de zero.

$$
M=\left[\begin{array}{ccccc}
-6 & -1 & 0 & -3 & 0 \\
-2 & 8 & 0 & 0 & 0 \\
0 & 7 & 0 & -6 & -2 \\
4 & 0 & -2 & 9 & 0 \\
0 & 1 & 0 & 0 & -1
\end{array}\right]
$$

Neste formato, todos valores diferentes de zero são armazenados de forma contínua, linha por linha. As colunas representas a posição referente a coluna original da matriz e o índice de linha representa a quantidade de elementos não-nulos na linha somados aos anteriores. Dessa forma, o tamanho dos vetores que contém os valores e a coluna é igual a quantidade de elementos não nulos da matriz e, por sua vez, a dimensão do vetor índice de linha tem a dimensão da matriz $n+1$. A tabela abaixo descreve a matriz $M$ no formato CSR.

Tabela 7.2: Exemplo da matriz $M$ no formato CSR

\begin{tabular}{l|c|c|c|c|c|c|c|c|c|c|c|c|c|c}
\hline Valores & $=$ & $(-6$ & -1 & -3 & -2 & 8 & 7 & -6 & -2 & 4 & -2 & 9 & 1 & $-1)$ \\
\hline colunas & $=$ & $(1$ & 2 & 4 & 1 & 2 & 2 & 4 & 5 & 1 & 3 & 4 & 2 & $5)$ \\
\hline índice linha & $=$ & $(1$ & 4 & 6 & 9 & 12 & $14)$ & & & & & & & \\
\hline
\end{tabular}




\section{Referências Bibliográficas}

AMESTOY, P. R.; DAVIS, T. A.; DUFF, I. S. An approximate minimum degree ordering algorithm. SIAM J. on Matrix Analysis and Applications, v. 17, p. 886-905, 1996.

AMESTOY, P. R. et al. A fully asynchronous multifrontal solver using distributed dynamic scheduling. SIAM J. Matrix Anal. Appl., Society for Industrial and Applied Mathematics, v. 23, n. 1, p. 15-41, 2001.

AMESTOY, P. R. et al. Hybrid scheduling for the parallel solution of linear systems. Parallel Computing, v. 2, p. 136-156, 2006.

BERTOLOTTI, F.; HERBET, T. Analysis of the stability of compressible boundary layer using PSE. Theor. Comp. Fluid Dyn., v. 3, p. 117-124, 1991.

BOYD, J. P. Lecture Notes in Engineering 49: Chebyshev and Fourier Spectral Methods. [S.l.]: Springer, 1989.

CANUTO, C. et al. Spectral Methods in Fluid Dynamics. [S.l.]: Springer, 1988.

CHOMAZ, J. M. Global instabilities in spatially developing flows: non-normality and nonlinearity. Annu. Rev. Fluid Mech., v. 37, p. 357-392, 2005.

CHOMAZ, J. M.; HUERRE, P.; REDEKOPP, L. G. Bifurcation to local and global modes in spatially developing flows. Phys. Re. Lett., v. 60, p. 25-28, 1988.

COHEN, C. B.; RESHOTKO, E. Similar solutions for the compressible laminar boundary layer with heat transfer and pressure gradient. NACA. Report, v. 1293, 1956.

COLONIOUS, T.; LELE, S. . K. Computacional aeroacoustics: progress on nonlinear problems of sound genereation. Progress in Aerospace Sciences, v. 40, p. 345-416, 2004.

CRIMINALE, W. O.; JACKSON, T. L.; JOSLIN, R. D. Theory and Computation in hydrodynamic stability. [S.l.]: Cambridge University Press, 2003.

CROUCH, J.; GARBARUK, A.; MAGIDOV, D. Predicting the onset of flow unsteadiness based on global instability. Journal of Computational Physics, v. 224, n. 2, p. $924-940$, 2007.

CROUCH, J. D.; GARBARUK, A.; MAGIDOV, D. Predicting the onset of flow unsteadiness based on global instability. J. Comp. Phys., v. 224, p. 924-940, 2007.

CROUCH, J. D. et al. Origin of transonic buffet on aerofoils. J. Fluid Mech., v. 628, p. $357-369,2009$.

DAVIO, M. Kronecker products and shuffle algebra. IEEE Transactions on Computers, v. 30, p. 116-125, 1981. 
DING, Y.; KAWAHARA, M. Linear stability of incompressible flow using a mixed finite element method. J. Comp. Phys., v. 139, n. 2, p. 243 - 273, 1998.

DRAZIN, P. G.; REID, W. H. Hydrodynamic stability. [S.l.]: Cambridge University Press, 1981.

DUC, A. L.; SESTERHENN, J.; FRIEDRICH, R. Instabilities in compressible attachment-line boundary layers. Phys. Fluids, v. 18, 2006.

EHRENSTEIN, U. On the linear stability of channel flows over riblets. Phys. Fluids, v. 8, p. $3194-3196,1996$.

GALLETTI, B.; BOTTARO, A. Large-scale secondary structures in duct flow. J. Fluid Mech., v. 512, p. 85-94, 2004.

GAVRILAKIS, S. Numerical simulation of low-reynolds-number turbulent flow through a straight square duct. J. Fluid Mech., v. 244, p. 101-129, 1992.

GENNARO, E. M. Análise da instabilidade hidrodinâmica de uma esteira assimétrica. Dissertação (Mestrado) — Universidade de São Paulo, 2008.

GENNARO, E. M. et al. Sparse techniques in global flow instability with application to compressible leading-edge flow. AIAA Journal, (submitted for publication), 2011.

GENNARO, E. M. et al. Linear instability of orthogonal compressible leading-edge boundary layer flow. AIAA-paper, n. 2011-3751, 2011.

GIANNETTI, F.; LUCHINI, P. Structural sensitivity of the first instability of the cylinder wake. J. Fluid Mech., v. 581, p. 167 - 197, 2007.

Görtler, H. Dreidimensionale Instabilität der ebenen Staupunktströmung gegenüber wirbelartigen Störungen. In: Görtler, H.; TOLLMIEN, W. (Ed.). 50 Jahre Grenzschichtforschung. [S.1.]: Vieweg und Sohn, 1955. p. 304-314.

HALL, P.; MALIK, M. R.; POLL, D. I. A. On the stability of an infinitive swept attachment line boundary layer. Proc. R. Soc. Lond. A, v. 395, p. 229-245, 1984.

HÄMMERLIN, H. Zur Instabilitätstheorie der ebenen Staupunktströmung, 50 jahre grenzschichtforschung. Vieweg und Sohn, p. 315-327, 1955.

HERBET, T. Parabolized stability equations. Annu. Rev. Fluid Mech., v. 29, p. 245-283, 1997.

HERMANNS, M.; HERNANDEZ, J. A. Stable high-order finite-difference methods based on non-uniform grid point distributions. Int. J. Numer. Meth. Fluids, v. 56, n. 3, p. 233-255, 2008.

HERNÁNDEZ, V. et al. A Survey of Software for Sparse Eigenvalue Problem. [S.l.], 2007.

HIEMENZ, K. Die grenzschicht an einem in den gleichförmigen flüssigkeitsstrom eingetauchten geraden kreiszylinder. Dingl. Polytechn. J., v. 326, p. 321-324, 1911. Thesis, Göttingen. 
HUERRE, P.; MONKEWITZ, P. A. Local and global instabilities in spatially developing flows. Annu. Rev. Fluid Mech., v. 22, p. 473-573, 1990.

KARYPIS, G.; KUMAR, V. METIS - A software package for partitioning unstructured graphs, partitioning meshes, and computing fill-reducing orderings of sparse matrices version 4.0. University of Minnesota, 1998.

KITSIOS, V. et al. BiGlobal stability analysis in curvilinear coordinates of massively separated lifting bodies. J. Comp. Phys., v. 228, n. 19, p. 7181-7196, 2009.

LEHOUCQ, R. B.; SALINGER, A. G. Large-scale eigenvalue calculations for stability analysis of steady flows on massively parallel computers. Int. J. Numer. Meth. Fluids, v. 36, p. $309-327,2001$.

LI, F.; MALIK, M. R. On the nature of PSE approximation. Theor. Comp. Fluid Dyn., v. 8, p. 253-273, 1996.

LIN, R. S.; MALIK, M. R. On the stability of attachment-line boundary layers. Part 1. The incompressible swept Hiemenz flow. J. Fluid Mech., v. 311, p. 239-255, 1996.

LIN, R. S.; MALIK, M. R. On the stability of attachment-line boundary layers. Part 2. The effect of leading-edge curvature. J. Fluid Mech., v. 333, p. 125-137, 1997.

MACK, C. J.; SCHMID, P. J. Direct numerical study of hypersonic flow around a swept parabolic body. v. 39, p. 1932-1943, 2010.

MACK, C. J.; SCHMID, P. J. A preconditioned Krylov technique for global hydrodynamic stability analysis of large-scale compressible flows. J. Comp. Phys., v. 229, n. 3 , p. $541-560,2010$.

MACK, C. J.; SCHMID, P. J. Global stability of swept flow around a parabolic body: features of the global spectrum. J. Fluid Mech., v. 669, p. 375-396, 2011.

MACK, C. J.; SCHMID, P. J.; SESTERHENN, J. L. Global stability of swept flow around a parabolic body: connecting attachment-line and crossflow modes. J. Fluid Mech., v. 611, p. 205-214, 2008.

MACK, L. M. Boundary layer linear stability theory. In: AGARD-R-709. [S.l.: s.n.], 1984. p. 3.1-3.81.

MEDEIROS, M. A. F. Nonlinear hydrodynamic instability. In: II school of transition and turbulence. Uberlândia - Brazil: [s.n.], 2000. p. 312-357. In portuguese.

MEDEIROS, M. A. F. Instabilidade hidrodinâmica não linear. In: Silveira Neto, A.; MANSUR, S. S. (Ed.). Turbulência. [S.l.]: ABCM, 2003. v. 2, p. 305-355.

MENDONÇA, M. T. Estabilildade de escoamentos laminares: teoria linear. In: Silveira Neto, A.; MANSUR, S. S. (Ed.). Turbulência. [S.l.]: ABCM, 2003. v. 2, p. 245-304.

NATARAJAN, R. An Arnoldi-based iterative scheme for nonsymmetric matrix pencils arising in finite element stability problems. J. Comp. Phys., v. 100, n. 1, p. $128-142$, 1992. 
NATARAJAN, R.; ACRIVOS, A. The instability of the steady flow past spheres. $J$. Fluid Mech., v. 254, p. 323 - 344, 1993.

NAYAR, N.; ORTEGA, J. M. Computation of selected eigenvalues of generalized eigenvalue problems. J. Comp. Phys., v. 108, n. 1, p. 8 -14, 1993.

OBRIST, D.; SCHMID, P. J. On the linear stability of swept attachment-line boundary layer flow. part 1. spectrum and asymptotic behaviour. J. Fluid Mech., v. 493, p. 1-29, 2003.

ORR, W. M. F. The stability or instability of the steady motions of a perfect liquid and of a viscous liquid. Part I: A perfect liquid; Part II: A viscous liquid. Proc. Roy. Irish. Acad., v. 27, p. 9-68 and 69-138, 1907.

RODRÍGUEZ, D.; THEOFILIS, V. Massively parallel numerical solution of the BiGlobal linear instability eigenvalue problem using dense linear algebra. AIAA J., v. 47, n. 10, p. 2449-2459, 2009.

ROSENHEAD, L. Laminar Boundary Layers. [S.1.]: Oxford University Press, 1963.

SAAD, Y. Variations on Arnoldi's method for computing eigenelements of large unsymmetric matrices. Linear Algebra and its Applications, v. 34, p. 269 - 295, 1980.

SAAD, Y. Iterative methods for sparse linear systems. [S.1.]: SIAM, 2000.

SCHMID, P.; HENNINGSON, D. Stability and transition in shear flows. [S.1.]: Springer, 2001.

SCHULZE, J. Towards a tighter coupling of bottom-up and top-down sparse matrix ordering methods. BIT Numerical Mathematics, Springer Netherlands, v. 41, p. 800-841, 2001.

SHANKAR, P. N.; DESHPANDE, M. D. Fluid mechanics in the driven cavity. Annu. Rev. Fluid Mech., v. 32, p. 93 - 136, 2000.

SIPP, D.; LEBEDEV, A. Global stability of base and mean flows: a general approach and its applications to cylinder and open cavity flows. J. Fluid Mech., v. 593, p. 333 $358,2007$.

SOMMERFELD, A. Ein Beitrag zur hydrodynamischen Erkärung der turbulnten

Flüssigkeitsbewegunen. In: Atti. del Congr. Internat. dei Mat. III. Roma: [s.n.], 1908. p. 116-124.

TATSUMI, T.; YOSHIMURA, T. Stability of the laminar flow in a rectangular duct. J. Fluid Mech., v. 212, p. 437-449, 1990.

THEOFILIS, V. Numerical experiments on the stability of leading-edge boundary-layer flow: a 2-dimensional linear study. Int. J. Numer. Meth. Fluids, v. 16, n. 2, p. 153-170, 1993.

THEOFILIS, V. Spatial stability of incompressible attachment-line flow. Theor. Comp. Fluid Dyn., v. 7, n. 3, p. 159-171, 1995. 
THEOFILIS, V. Globally-unstable flows in open cavities. AIAA PAPER, n. 2000-1965, 2000.

THEOFILIS, V. Advances in global linear instability of nonparallel and three-dimensional flows. Prog. Aero. Sciences, v. 39 (4), p. 249-315, 2003.

THEOFILIS, V. Global linear instability. Annu. Rev. Fluid Mech., v. 43, p. 319-352, 2011.

THEOFILIS, V.; DUCK, P.; OWEN, J. Viscous linear stability analysis of rectangular duct and cavity flows. J. Fluid Mech., v. 505, p. 249-286, 2006.

THEOFILIS, V. et al. The extended Görtler-Hämmerlin model for linear instability of three-dimensional incompressible swept attachment-line boundary layer flow. J. Fluid Mech., v. 487, p. 271-313, 2003.

THEOFILIS, V.; FEDOROV, A. V.; COLLIS, S. S. Leading-edge boundary layer flow Prandtl's vision, current developments and future perspectives. IUTAM Symposium on One Hundred Years of Boundary Layer Research, v. 129, 2004.

TREFETHEN, L. N. Spectral elements in Matlab. [S.l.]: SIAM, 2000. 